\title{
The second INTEGRAL AGN catalogue
}

\author{
V. Beckmann ${ }^{1,2,3}$, S. Soldi ${ }^{4}$, C. Ricci ${ }^{1,2}$, J. Alfonso-Garzón ${ }^{5}$, T. J.-L. Courvoisier ${ }^{1,2}$, A. Domingo ${ }^{5}$, N. Gehrels ${ }^{6}$, \\ P. Lubiński ${ }^{7,1}$, J. M. Mas-Hesse ${ }^{5}$, and A. A. Zdziarski ${ }^{7}$
}

1 ISDC Data Centre for Astrophysics, Chemin d'Écogia 16, 1290 Versoix, Switzerland e-mail: beckmann@apc.univ-paris7.fr

2 Observatoire Astronomique de l'Université de Genève, Chemin des Maillettes 51, 1290 Sauverny, Switzerland

3 APC Laboratory, Université Paris Diderot, 10 rue A. Domon et L. Duquet, 75205 Paris Cedex 13, France

${ }^{4}$ Laboratoire AIM - CNRS - CEA/DSM - Université Paris Diderot (UMR 7158), CEA Saclay, DSM/IRFU/SAp, 91191 Gif-sur-Yvette, France

5 Centro de Astrobiología LAEX (CSIC-INTA), POB 78, 28691 Villanueva de la Cañada, Madrid, Spain

6 Astrophysics Science Division, NASA Goddard Space Flight Center, Code 661, MD 20771, USA

7 Centrum Astronomiczne im. M. Kopernika, Bartycka 18, 00-716 Warszawa, Poland

Received 19 March 2009 / Accepted 30 June 2009

\section{ABSTRACT}

\begin{abstract}
Aims. The INTEGRAL mission provides a large data set for studying the hard X-ray properties of AGN and allows testing of the unified scheme for AGN

Methods. We present analysis of INTEGRAL IBIS/ISGRI, JEM-X, and OMC data for 199 AGN supposedly detected by INTEGRAL above $20 \mathrm{keV}$.

Results. The data analysed here allow significant spectral extraction on 148 objects and an optical variability study of 57 AGN. The slopes of the hard X-ray spectra of Seyfert 1 and Seyfert 2 galaxies are found to be consistent within the uncertainties, whereas higher cut-off energies and lower luminosities we measured for the more absorbed/type 2 AGN. The intermediate Seyfert 1.5 objects exhibit hard X-ray spectra consistent with those of Seyfert 1 . When applying a Compton reflection model, the underlying continua appear the same in Seyfert 1 and 2 with $\Gamma \simeq 2$, and the reflection strength is about $R \simeq 1$, when assuming different inclination angles. A significant correlation is found between the hard X-ray and optical luminosity and the mass of the central black hole in the sense that the more luminous objects appear to be more massive. There is also a general trend toward the absorbed sources and type 2 AGN having lower Eddington ratios. The black hole mass appears to form a fundamental plane together with the optical and X-ray luminosity of the form $L_{V} \propto L_{\mathrm{X}}^{0.6} M_{\mathrm{BH}}^{0.2}$, similar to what is found between $L_{R}, L_{\mathrm{X}}$, and $M_{\mathrm{BH}}$.

Conclusions. The transition from the type 1 to type 2 AGN appears to be smooth. The type 2 AGN are less luminous and have less accreting super massive black holes. The unified model for Seyfert galaxies seems to hold, showing in hard X-rays that the central engine is the same in Seyfert 1 and 2, but seen under different inclination angles and absorption. The fundamental plane links the accretion mechanism with the bulge of the host galaxy and with the mass of the central engine in the same way in all types of Seyfert galaxies.
\end{abstract}

Key words. galaxies: active - galaxies: Seyfert - X-rays: galaxies - surveys - catalogs

\section{Introduction}

The extragalactic X-ray sky is dominated by active galactic nuclei (AGN), which are commonly assumed to host an accreting supermassive black hole in the centres of galaxies. X-ray spectroscopy has been vital in the study of the AGN phenomenon, because it probes the condition of matter in the vicinity of the black hole. One model for the X-ray emission is that of a hot corona lingering on top of the inner accretion disc of the black hole and emitting inverse Compton radiation from disc photons that have been upscattered by energetic electrons. Another model assumes a disc with a hot inner advection-dominated accretion flow (ADAF; e.g. Abramowicz et al. 1996). An alternative model for the accretion process onto black holes is that of clumpy accretion flows (e.g. Guilbert \& Rees 1988). Courvoisier \& Türler (2005) assume that the different elements (clumps) of the

* All tables of this paper are also available in electronic form at the CDS via anonymous ftp to cdsarc.u-strasbg.fr (130.79.128.5) or via

http://cdsweb.u-strasbg.fr/cgi-bin/qcat?J/A+A/505/417 accretion flow have velocities that may differ substantially. As a consequence, collisions between these clumps will appear when the clumps are close to the central object, resulting in radiation.

Because optical spectroscopy distinguishes between two main types of low-luminosity AGN, the broad-line Seyfert 1 and narrow-line Seyfert 2 objects, a similar distinction is apparent between unabsorbed sources with on-average softer $\mathrm{X}$-ray spectra and the flatter spectra of absorbed sources. This has been noticed by Zdziarski et al. (1995), based on Ginga and CGRO/OSSE data and later confirmed e.g. by Gondek et al. (1996) using combined EXOSAT, Ginga, HEAO-1, and CGRO/OSSE spectra, and by Beckmann et al. (2006) using INTEGRAL IBIS/ISGRI data of AGN above $20 \mathrm{keV}$. A study of BeppoSAX PDS spectra of 45 Seyfert galaxies has come to a similar conclusion, although the spectra of Seyfert 2 appeared steeper when considering a possible cut-off in the spectra of Seyfert 1 galaxies (Deluit \& Courvoisier 2003). X-ray data already show that most, but not all, AGN unabsorbed in the X-rays are Seyfert 1 type, and most, but not all, AGN that are absorbed belong to the Seyfert 2 group (e.g. Awaki et al. 1991). 
Thus a longstanding discussion has been, whether these two groups indeed represent physically different types of objects, or whether they can be unified under the assumption that they are intrinsically the same but seen from a different viewing angle with respect to absorbing material in the vicinity of the central engine (e.g. Antonucci 1993), and that the difference in X-ray spectral slope can be explained solely by the absorption and reflection components. This unified model naturally explains the different Seyfert types in a way that the broad-line region is either visible (Seyfert 1) or hidden (Seyfert 2) possibly by the same material in the line of sight as is responsible for the absorption detectable at soft X-rays (e.g. Lawrence \& Elvis 1982). On the other hand, the model has some problems explaining other aspects of AGN, for example, that some Seyfert galaxies change their type from 1 to 2 and back, but also the observation that Seyfert 2 objects exhibit flatter hard X-ray spectra than Seyfert 1 even in the energy range $>20 \mathrm{keV}$, where absorption should not play a major role unless $N_{\mathrm{H}} \gg 10^{24} \mathrm{~cm}^{-2}$. Also, the existence of Seyfert 2 galaxies that show no absorption in the soft X-rays, like NGC 3147 and NGC 4698 (Pappa et al. 2001) cannot be explained by the unified model.

Lately, two hard X-ray missions have provided surveys at $>20 \mathrm{keV}$ with enough sky coverage to be suitable for population studies of AGN. One is the NASA-led Swift mission (Gehrels et al. 2004) launched in 2004, the other one the ESA-led INTEGRAL satellite (Winkler et al. 2004), launched in October 2002. Due to its observation strategy of followingup gamma-ray bursts, Swift/BAT (Barthelmy et al. 2005) provides a more homogeneous sky coverage in the $15-195 \mathrm{keV}$ energy range, while the hard X-ray imager IBIS/ISGRI onboard INTEGRAL is more sensitive and extends up to several hundred $\mathrm{keV}$ with better spectral resolution. INTEGRAL provides broad-band coverage through the additional X-ray monitor JEM-X in the 3-30 keV range (Lund et al. 2003) and provides photometry with the optical camera OMC in the $V$-band (Mas-Hesse et al. 2003).

The AGN surveys provided by Swift/BAT (Tueller et al. 2008) and INTEGRAL IBIS/ISGRI (Beckmann et al. 2006b; Bassani et al. 2007) have already led to the discovery that the fraction of absorbed and Compton thick sources is less than expected from cosmic X-ray background synthesis models (e.g. Treister \& Urry 2005; Gilli et al. 2007). With the ongoing INTEGRAL mission, it is now possible to compile a large sample of AGN for spectroscopic and correlation studies and to probe the unified model for AGN. The data analysis is described in Sect. 2, the average properties of the AGN in the sample in Sect. 3, the discussion of the properties in the view of unified models in Sect. 4, and we end with the conclusions in Sect. 5. Notes on individual sources can be found in the Appendix (Sect. A).

\section{Data analysis}

The list of AGN presented here is based on all INTEGRAL detections of AGN reported in the literature, therefore enter into the INTEGRAL general reference catalogue ${ }^{1}$ (Ebisawa et al. 2003; Bodaghee et al. 2007). It has to be pointed out that for many sources, we present the first INTEGRAL spectral analysis, because Bodaghee et al. (2007), Sazonov et al. (2007), and Bassani et al. (2006) did not include spectral analysis, and Beckmann et al. (2006) discussed a sample of 38 AGN based only on

${ }^{1}$ For the latest version of the catalogue see http://isdc.unige. ch/index.cgi?Data+catalogs

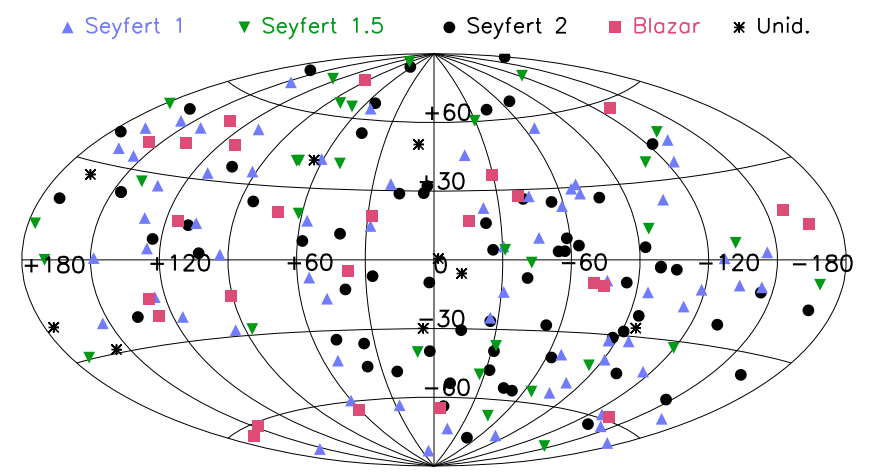

Fig. 1. INTEGRAL-detected AGN during the first 5 years of the mission. As unidentified we mark those sources where the AGN type has not been determined yet.

1.3 years of INTEGRAL data. With the INTEGRAL mission continuing smoothly, most of the sky has been observed in the first 5 years of operations, leading to a rather uniform sky distribution of detected AGN, as shown in Fig. 1. For each extragalactic source, we analysed the IBIS/ISGRI, JEM-X, and OMC data from the early mission (revolution 26 starting on 30 December 2002) up to spacecraft revolution 530 (ending on 17 February 2007), covering more than 4 years of data. To include only high-quality data, the selection considers ISGRI data taken at an off-axis angle smaller than $10^{\circ}$ and includes only those observations that lasted for at least 500 s. Analysis software used in this work is version 7 of the Offline Standard Analysis Software (OSA) provided by the ISDC Data Centre for Astrophysics (Courvoisier et al. 2003). For each source, imaging analysis was performed to determine the significant sources in the field around the AGN. Taking their fluxes into account is important when analysing data from coded-mask instruments, because all sources in the field add to the background of the source of interest. Then standard spectral extraction was used, considering all significant sources in the field.

For the X-ray monitor JEM-X, a similar selection of data was performed, using a maximum off-axis angle of $3^{\circ}$ due to the smaller field of view compared to IBIS. The much lower effective exposure time when compared to IBIS/ISGRI (Table 1) results in only 23 detections of AGN by JEM-X with a significance $>5 \sigma$. For JEM-X, spectra were extracted from the mosaic images, since this procedure is more reliable for faint sources than the standard spectral extraction in OSA 7.

Naturally, some sources reported in the literature do not show up significantly in the data analysed here, because they were observed after February 2007, or they are, like the blazar class, highly variable and therefore do not give a significant detection in the combined data set. The 199 AGN reported to be found in INTEGRAL data are listed in Table 1, together with their redshift, position (J2000.0), and their effective exposure time in IBIS/ISGRI and JEM-X for the data set used here. Twelve sources, which were reported in the literature but gave a detection significance $<3 \sigma$ in the data presented here are listed in Table 1 and marked by an $x$. These objects are not considered in the following analysis. All errors given in this paper are at the $1 \sigma$ level.

\subsection{Black hole masses}

We also include in Table 1 the black hole masses of the central engine and the method used to determine them, as found in the literature. Different methods can be used to estimate the mass of 
Table 1. INTEGRAL AGN catalogue. The column Method indicates the method used to determine the black hole mass, see Sect. 2.1 for details.

\begin{tabular}{|c|c|c|c|c|c|c|c|c|}
\hline Name & Type & $z$ & $\begin{array}{r}\text { RA } \\
\text { [deg] }\end{array}$ & $\begin{array}{r}\text { Dec } \\
\text { [deg] }\end{array}$ & $\begin{array}{r}\text { ISGRI } \\
{[\mathrm{ks}]}\end{array}$ & $\begin{array}{r}\text { JEM-X } \\
{[\mathrm{ks}]}\end{array}$ & $\begin{array}{c}\log M_{\mathrm{BH}} \\
{\left[M_{\odot}\right]}\end{array}$ & $\overline{\text { Method }}$ \\
\hline IGR J00040+7020 & Sy2 & 0.096 & 1.00638 & 70.32125 & 1947.3 & 20.9 & & \\
\hline IGR J00254+6822 & Sy2 & 0.012 & 6.38092 & 68.36147 & 2534.6 & 178.9 & & \\
\hline IGR J00335+6126 & Sy1 & 0.105 & 8.3265 & 61.46178 & 3338.7 & 356.5 & $8.5 \pm 0.5^{a}$ & LL/CL \\
\hline 1ES $0033+59.5$ & BLLac & 0.086 & 8.96929 & 59.83461 & 3338.7 & 356.4 & & \\
\hline Mrk 348 & Sy2 & 0.0151 & 12.19642 & 31.95697 & 150.7 & 21.1 & $7.2 \pm 0.7^{b}$ & SO \\
\hline NGC $418^{x}$ & AGN & 0.0190 & 17.64842 & -30.22128 & 38.5 & - & & \\
\hline NGC 526A & Sy1.5 & 0.0191 & 20.97583 & -35.06528 & 73.7 & - & $8.1 \pm 0.7^{c}$ & $\mathrm{~S}$ \\
\hline ESO 297-18 & Sy2 & 0.0252 & 24.65492 & -40.01131 & 59.5 & - & $9.7 \pm 0.5^{n}$ & KM \\
\hline IGR J01528-0326 & Sy2 & 0.0167 & 28.20375 & -3.44749 & 837.1 & 79.6 & & \\
\hline NGC 788 & Sy 2 & 0.0136 & 30.27687 & -6.81553 & 926.6 & 117.9 & $7.5 \pm 0.7^{b}$ & SO \\
\hline Mrk 590 & Sy1.2 & 0.0264 & 33.63984 & -0.76669 & 936.0 & 266.6 & $7.14_{-0.09}^{+0.1 d}$ & $\mathrm{R}$ \\
\hline IGR J02097+5222 & Sy1 & 0.0492 & 32.40700 & 52.44543 & 698.4 & 2.0 & & \\
\hline SWIFT J0216.3+5128 & Sy 2 & 0.0288 & 34.11292 & 51.42375 & 481.9 & 2.0 & & \\
\hline Mrk 1040 & Sy1.5 & 0.0167 & 37.06079 & 31.31094 & 42.8 & - & $7.6 \pm 0.3^{e}$ & $\mathrm{~S}$ \\
\hline IGR J02343+3229 & Sy2 & 0.0162 & 38.57500 & 32.48333 & 70.5 & - & & \\
\hline NGC 985 & Sy1 & 0.0431 & 38.65738 & -8.78761 & 808.1 & 48.3 & $8.9 \pm 0.5^{n}$ & KM \\
\hline NGC 1052 & Sy2 & 0.0050 & 40.27000 & -8.25578 & 707.0 & 31.1 & $8.2 \pm 0.3^{e}$ & $\mathrm{~S}$ \\
\hline RBS 345 & Sy1 & 0.0690 & 40.56667 & 5.53000 & 443.2 & - & & \\
\hline NGC 1068 & Sy2 & 0.0288 & 40.67012 & -0.01344 & 914.4 & 44.8 & $7.2 \pm 0.1^{e}$ & M \\
\hline QSO B0241+62 & Sy1 & 0.0446 & 41.24042 & 62.46847 & 711.9 & 35.2 & & \\
\hline IGR J02466-4222 & AGN & 0.0695 & 41.65375 & -42.36600 & 191.4 & 21.9 & & \\
\hline IGR J02501+5440 & Sy2 & 0.015 & 42.67417 & 54.70419 & 773.8 & 13.5 & & \\
\hline MCG-02-08-014 & Sy2 & 0.0168 & 43.09750 & -8.51042 & 528.1 & 4.7 & & \\
\hline NGC 1142 & Sy2 & 0.0288 & 43.80133 & -0.18381 & 634.1 & - & $9.4 \pm 0.5^{n}$ & KM \\
\hline QSO B0309+411 & Sy1 & 0.136 & 48.25817 & 41.33366 & 488.4 & 303.1 & & \\
\hline IGR J03184-0014 ${ }^{x}$ & QSO & - & 49.60000 & -0.22889 & - & - & & \\
\hline NGC 1275 & Sy2 & 0.0176 & 49.95067 & 41.51170 & 506.8 & 282.0 & $8.5 \pm 0.7^{e}$ & $\mathrm{~S}$ \\
\hline $1 \mathrm{H} 0323+342$ & Sy1 & 0.0629 & 51.17150 & 34.17941 & 431.6 & 0.9 & & \\
\hline IGR J03334+3718 & Sy 1.5 & 0.0547 & 53.32833 & 37.30305 & 463.4 & 5.6 & & \\
\hline NGC 1365 & Sy1.5 & 0.0055 & 53.40208 & -36.13806 & 143.3 & 3.0 & $7.7 \pm 0.3^{f}$ & $\mathrm{~S}$ \\
\hline IGR J03532-6829 & BLLac & 0.0870 & 58.30833 & -68.48306 & 740.8 & 9.4 & & \\
\hline $3 \mathrm{C} 111$ & Sy1 & 0.0485 & 64.58867 & 38.02661 & 160.1 & - & $9.6 \pm 0.8^{c}$ & B \\
\hline $3 \mathrm{C} 120$ & Sy1 & 0.0330 & 68.29623 & 5.35434 & 365.9 & 79.3 & $7.7 \pm 0.2^{g}$ & $\mathrm{R}$ \\
\hline UGC 3142 & Sy1 & 0.0217 & 70.94537 & 28.97194 & 449.6 & 39.9 & & \\
\hline LEDA 168563 & Sy1 & 0.0290 & 73.01958 & 49.54583 & 112.5 & - & & \\
\hline ESO 33-2 & Sy 2 & 0.0181 & 73.99834 & -75.54056 & 1110.4 & - & & \\
\hline $4 \mathrm{U} 0517+17$ & Sy 1.5 & 0.0179 & 77.68958 & 16.49861 & 1145.4 & 19.8 & & \\
\hline Ark 120 & Sy1 & 0.0327 & 79.04784 & -0.15017 & 571.9 & 63.1 & $8.18^{+0.6}$ & $\mathrm{R}$ \\
\hline IGR J05270-6631 ${ }^{x}$ & QSO & 0.978 & 81.56021 & -66.5125 & 631.7 & 323.4 & $8.4 \pm 0.5^{a}$ & $\mathrm{LL} / \mathrm{CL}$ \\
\hline PKS $0528+134^{x}$ & blazar & 2.060 & 82.73507 & 13.53199 & 917.6 & 157.3 & & \\
\hline NGC 2110 & Sy2 & 0.0078 & 88.04742 & -7.45622 & 20.5 & - & $8.3 \pm 0.3^{e}$ & $\mathrm{~S}$ \\
\hline MCG+08-11-011 & Sy1.5 & 0.0205 & 88.72338 & 46.43934 & 50.8 & - & $8.1 \pm 0.6^{c}$ & SO \\
\hline IRAS $05589+2828$ & Sy1 & 0.0330 & 90.54042 & 28.47139 & 1689.9 & 29.0 & & \\
\hline SWIFT J0601.9-8636 & Sy2 & 0.0064 & 91.41292 & -86.63111 & 177.8 & 31.9 & $7.9 \pm 0.5^{n}$ & KM \\
\hline IGR J06117-6625 & Sy 1.5 & 0.230 & 92.95208 & -66.40847 & 1122.2 & 97.2 & & \\
\hline Mrk 3 & Sy2 & 0.0135 & 93.90129 & 71.03748 & 814.9 & 55.4 & $8.7 \pm 0.3^{e}$ & $\mathrm{~S}$ \\
\hline IGR J06239-6052 & Sy2 & 0.0405 & 95.94004 & -60.97927 & 598.0 & - & & \\
\hline IGR J06292+4858 & BLLac & 0.097 & 97.300 & 48.97389 & - & - & & \\
\hline PKS 0637-752 & Sy1 & 0.651 & 98.94379 & -75.27133 & 1066.6 & 22.7 & $9.4 \pm 0.5^{e}$ & CL \\
\hline Mrk 6 & Sy 1.5 & 0.0188 & 103.0513 & 74.42689 & 819.4 & 109.7 & $8.2 \pm 0.5^{n}$ & KM \\
\hline QSO B0716+714 & BLLac & 0.3 & 110.4727 & 71.34343 & 863.8 & 162.8 & & \\
\hline LEDA 96373 & Sy2 & 0.0294 & 111.6096 & -35.90583 & 180.9 & 5.6 & & \\
\hline IGR J07437-5137 & Sy2 & 0.025 & 115.9208 & -51.61694 & 947.3 & - & & \\
\hline IGR J07565-4139 & Sy2 & 0.021 & 119.0817 & -41.62836 & 1703.0 & 50.6 & & \\
\hline IGR J07597-3842 & Sy1 & 0.040 & 119.9242 & -38.73223 & 1288.1 & 31.8 & $8.3 \pm 0.5^{p}$ & LL \\
\hline ESO 209-12 & Sy 1.5 & 0.0405 & 120.4900 & -49.77833 & 2288.6 & 117.7 & & \\
\hline PG 0804+761 & Sy1 & 0.10 & 122.7444 & 76.04514 & 694.9 & 44.9 & $8.84_{-0.06}^{+0.05^{9}}$ & $\mathrm{R}$ \\
\hline Fairall 1146 & Sy 1.5 & 0.0316 & 129.6279 & -35.99306 & 1689.9 & 26.2 & & \\
\hline QSO B0836+710 & BLLac & 2.1720 & 130.3515 & 70.89506 & 754.4 & 29.7 & & \\
\hline IGR J09026-4812 & Sy1 & 0.039 & 135.6555 & -48.22608 & 3773.3 & 470.1 & & \\
\hline SWIFT J0917.2-6221 & Sy1 & 0.0573 & 139.0392 & -62.32486 & 669.0 & 16.1 & & \\
\hline IGR J09253+6929 & Sy1 & 0.039 & 141.321 & 69.488 & 324.1 & - & $7.6 \pm 0.5^{a}$ & $\mathrm{LL} / \mathrm{CL}$ \\
\hline Mrk 110 & NLS1 & 0.0353 & 141.3036 & 52.28625 & 54.2 & - & $7.42_{-01}^{+0.09 g}$ & $\mathrm{R}$ \\
\hline IGR J09446-2636 & Sy 1.5 & 0.1425 & 146.1500 & -26.60000 & 157.1 & - & & \\
\hline NGC 2992 & Sy1 & 0.0077 & 146.4252 & -14.32639 & 383.3 & 66.7 & $7.7 \pm 0.3^{e}$ & $\mathrm{~S}$ \\
\hline
\end{tabular}


Table 1. continued.

\begin{tabular}{|c|c|c|c|c|c|c|c|c|}
\hline Name & Type & $z$ & $\begin{array}{r}\mathrm{RA} \\
{[\mathrm{deg}]} \\
\end{array}$ & $\begin{array}{r}\text { Dec } \\
{[\mathrm{deg}]}\end{array}$ & $\begin{array}{r}\text { ISGRI } \\
{[\mathrm{ks}]} \\
\end{array}$ & $\begin{array}{r}\text { JEM-X } \\
{[\mathrm{ks}]} \\
\end{array}$ & $\begin{array}{c}\log M_{\mathrm{BH}} \\
{\left[M_{\odot}\right]} \\
\end{array}$ & Method \\
\hline MCG-05-23-016 & Sy2 & 0.0085 & 146.9173 & -30.94886 & 130.2 & - & $6.3 \pm 0.5^{c}$ & SO \\
\hline IGR J09523-6231 & Sy1.5 & 0.252 & 148.0854 & -62.54333 & 1059.1 & 111.1 & & \\
\hline NGC 3081 & Sy2 & 0.0080 & 149.8731 & -22.82628 & 255.3 & - & $7.4 \pm 0.3^{b}$ & $\mathrm{~S}$ \\
\hline SWIFT J1009.3-4250 & Sy2 & 0.033 & 152.4512 & -42.81222 & 437.4 & - & & \\
\hline IGR J10147-6354 & Sy1.2 & 0.202 & 153.6750 & -63.89194 & 1182.9 & 104.6 & $8.6 \pm 0.5^{a}$ & LL/CL \\
\hline NGC 3227 & Sy1.5 & 0.0039 & 155.8776 & 19.86492 & 142.0 & - & $7.3_{-0.1}^{+0.2 h}$ & K \\
\hline NGC 3281 & Sy2 & 0.0107 & 157.9669 & -34.85369 & 211.3 & 41.9 & $8.0 \pm 0.5^{n}$ & KM \\
\hline SWIFT J1038.8-4942 & Sy 1.5 & 0.060 & 159.6875 & -49.78194 & 908.6 & 10.2 & & \\
\hline IGR J10404-4625 & Sy2 & 0.0237 & 160.0928 & -46.42353 & 673.6 & - & & \\
\hline Mrk 421 & BLLac & 0.0300 & 166.1138 & 38.20883 & 826.1 & 482.4 & $8.3 \pm 0.3^{e}$ & $\mathrm{~S}$ \\
\hline IGR J11366-6002 & Sy2 & 0.014 & 174.1754 & -60.05217 & 2196.0 & 315.7 & & \\
\hline NGC 3783 & Sy1 & 0.0097 & 174.7574 & -37.73853 & 22.9 & - & $7.47_{-0.09}^{+0.07 g}$ & $\mathrm{R}$ \\
\hline IGR J12026-5349 & Sy2 & 0.028 & 180.6985 & -53.83547 & 1535.9 & 116.6 & & \\
\hline NGC 4051 & Sy1.5 & 0.0023 & 180.7901 & 44.53144 & 793.2 & - & $6.3 \pm 0.2^{g}$ & $\mathrm{R}$ \\
\hline NGC 4138 & Sy1.5 & 0.0030 & 182.3745 & 43.68500 & 798.4 & 31.4 & $6.8 \pm 0.5^{n}$ & KM \\
\hline NGC 4151 & Sy 1.5 & 0.0033 & 182.6364 & 39.40545 & 820.3 & 548.3 & $7.5_{-0.6}^{+0.1^{h}}$ & $\mathrm{~K}$ \\
\hline NGC 4180 & AGN & 0.0070 & 183.2627 & 7.03881 & 1016.8 & 72.1 & & \\
\hline Was 49 & Sy2 & 0.0610 & 183.5742 & 29.52872 & 931.4 & - & & \\
\hline Mrk 766 & Sy1.5 & 0.0129 & 184.6110 & 29.81267 & 994.6 & - & $6.5 \pm 0.3^{i}$ & $\mathrm{~S}$ \\
\hline NGC 4258 & Sy1.5 & 0.0015 & 184.7397 & 47.30397 & 816.2 & 4.6 & $7.59 \pm 0.01^{i}$ & M \\
\hline 4C 04.42 & BLLac & 0.9650 & 185.5940 & 4.22106 & 1277.5 & 232.6 & & \\
\hline Mrk 50 & Sy1 & 0.0234 & 185.8506 & 2.67911 & 1320.8 & 264.8 & & \\
\hline NGC 4388 & Sy2 & 0.0084 & 186.4455 & 12.66203 & 869.2 & 146.8 & $7.2 \pm 0.6^{b}$ & SO \\
\hline NGC 4395 & Sy1.5 & 0.0011 & 186.4539 & 33.54661 & 1103.5 & - & $4.7_{-0.7}^{+0.3 i}$ & $\mathrm{~V}$ \\
\hline $3 \mathrm{C} 273$ & QSO & 0.1583 & 187.2779 & 2.05239 & 2004.6 & 299.2 & $9.81_{-0.07}^{+0.1 k}$ & $\mathrm{R}$ \\
\hline NGC 4507 & Sy2 & 0.0118 & 188.9023 & -39.90925 & 389.3 & - & $7.6 \pm 0.6^{c}$ & SO \\
\hline SWIFT J1238.9-2720 & Sy2 & 0.0250 & 189.7271 & -27.30778 & 71.3 & - & $8.6 \pm 0.5^{n}$ & KM \\
\hline IGR J12391-1612 & Sy2 & 0.0367 & 189.7762 & -16.17975 & 618.7 & 39.4 & $8.9 \pm 0.5^{n}$ & KM \\
\hline NGC 4593 & Sy1 & 0.0090 & 189.9143 & -5.34425 & 1466.5 & 213.5 & $6.99_{-0.1}^{+0.0 h^{h}}$ & $\mathrm{R}$ \\
\hline IGR J12415-5750 & Sy1.5 & 0.0242 & 190.3575 & -57.83417 & 1653.1 & 101.6 & $8.0 \pm 0.5^{a}$ & LL/CL \\
\hline PKS 1241-399 & QSO & 0.1910 & 191.1223 & -40.21289 & 551.3 & - & & \\
\hline ESO 323-32 & Sy1 & 0.0160 & 193.3348 & -41.63717 & 744.4 & 23.9 & & \\
\hline 3C 279 & BLLac & 0.5362 & 194.0465 & -5.78931 & 1144.1 & 185.0 & $8.4 \pm 0.5^{e}$ & CL \\
\hline IGR J13000+2529 & AGN & - & 195.0000 & 25.48333 & 937.1 & 241.0 & & \\
\hline Mrk 783 & Sy 1.5 & 0.0672 & 195.7452 & 16.40763 & 728.2 & 11.2 & & \\
\hline IGR J13038+5348 & Sy1 & 0.0302 & 195.9975 & 53.79172 & 366.3 & 16.5 & $7.5 \pm 0.5^{n}$ & KM \\
\hline NGC 4945 & Sy 2 & 0.0019 & 196.3587 & -49.47083 & 1100.0 & 141.8 & $6.2 \pm 0.3^{l}$ & $\mathrm{M}$ \\
\hline IGR J13057+2036 & AGN & - & 196.4273 & 20.58103 & 853.1 & 12.0 & & \\
\hline ESO 323-77 & Sy1 & 0.0150 & 196.6108 & -40.41389 & 920.1 & 65.3 & $7.4 \pm 0.6^{c}$ & LL \\
\hline IGR J13091+1137 & Sy2 & 0.025 & 197.2733 & 11.63414 & 391.1 & 8.2 & $8.6 \pm 0.5^{n}$ & KM \\
\hline IGR J13109-5552 & Sy1 & 0.104 & 197.6795 & -55.86991 & 1506.6 & 26.6 & & \\
\hline NGC 5033 & Sy1.5 & 0.0029 & 198.3650 & 36.59358 & 743.2 & 6.2 & & \\
\hline IGR J13149+4422 & Sy2 & 0.0366 & 198.8155 & 44.40750 & 421.4 & 56.8 & & \\
\hline Cen A & Sy2 & 0.0018 & 201.3651 & -43.01911 & 1268.0 & 149.5 & $8.0 \pm 0.6^{c}$ & $\mathrm{~K}$ \\
\hline ESO 383-18 & Sy2 & 0.0124 & 203.3596 & -34.01631 & 756.8 & 191.6 & & \\
\hline MCG-06-30-015 & Sy1.2 & 0.0077 & 203.9741 & -34.29558 & 767.2 & 213.0 & $6.7_{-0.2}^{+0.1 i^{i}}$ & $\mathrm{~S}$ \\
\hline NGC 5252 & Sy2 & 0.0230 & 204.5667 & 4.54236 & 58.9 & - & $9.03_{-0.02}^{+0.4}$ & $\mathrm{~K}$ \\
\hline Mrk 268 & Sy 2 & 0.0399 & 205.2964 & 30.37811 & 728.7 & - & & \\
\hline $4 U$ 1344-60 & Sy 1.5 & 0.0129 & 206.8833 & -60.61000 & 1537.7 & 231.2 & & \\
\hline IC 4329A & Sy1 & 0.0161 & 207.3304 & -30.30956 & 381.9 & 52.6 & $\sim 7^{g}$ & $\mathrm{R}$ \\
\hline Circinus Galaxy & Sy2 & 0.0014 & 213.2871 & -65.34084 & 2272.1 & 201.3 & $6.04_{-0.09}^{+0.07}$ & M \\
\hline NGC 5506 & Sy1.9 & 0.0062 & 213.3120 & -3.20750 & 102.9 & 88.5 & $6.7 \pm 0.7^{b}$ & SO \\
\hline IGR J14175-4641 & Sy2 & 0.076 & 214.2664 & -46.69419 & 1268.8 & 21.4 & & \\
\hline NGC 5548 & Sy1.5 & 0.0172 & 214.4985 & 25.13706 & 200.9 & 42.4 & $7.82 \pm 0.02^{h}$ & $\mathrm{R}$ \\
\hline RHS 39 & Sy1 & 0.0222 & 214.8425 & -26.64472 & 454.4 & 8.1 & $8.7 \pm 0.5^{n}$ & KM \\
\hline H $1426+428$ & BLLac & 0.1291 & 217.1358 & 42.67472 & 499.5 & 224.7 & $9.1 \pm 0.7^{e}$ & SB \\
\hline IGR J14471-6414 & Sy1 & 0.053 & 221.6158 & -64.27319 & 1797.1 & 171.8 & & \\
\hline IGR J14471-6319 & Sy 2 & 0.038 & 221.8120 & -63.28868 & 1842.9 & 230.3 & & \\
\hline IGR J14492-5535 & AGN & - & 222.3038 & -55.60578 & 1886.0 & 142.4 & & \\
\hline IGR J14515-5542 & Sy2 & 0.018 & 222.8880 & -55.67733 & 1869.2 & 144.0 & & \\
\hline IGR J14552-5133 & NLS1 & 0.016 & 223.8223 & -51.57102 & 2007.3 & 7.9 & $6.3 \pm 0.5^{p}$ & LL \\
\hline IGR J14561-3738 & Sy2 & 0.024 & 224.0342 & -37.64803 & 2185.9 & 340.8 & & \\
\hline
\end{tabular}


Table 1. continued.

\begin{tabular}{|c|c|c|c|c|c|c|c|c|}
\hline Name & Type & $z$ & $\begin{array}{r}\mathrm{RA} \\
\text { [deg] }\end{array}$ & $\begin{array}{r}\mathrm{Dec} \\
\text { [deg] }\end{array}$ & $\begin{array}{r}\text { ISGRI } \\
{[\mathrm{ks}]}\end{array}$ & $\begin{array}{r}\text { JEM-X } \\
{[\mathrm{ks}]}\end{array}$ & $\begin{array}{c}\log M_{\mathrm{BH}} \\
{\left[M_{\odot}\right]}\end{array}$ & $\overline{\text { Method }}$ \\
\hline IGR J14579-4308 & Sy2 & 0.016 & 224.4296 & -43.13000 & 2012.9 & 333.4 & & \\
\hline Mrk 841 & Sy 1.5 & 0.0364 & 226.0050 & 10.43782 & 131.3 & 6.7 & $8.5 \pm 0.7^{c}$ & $\mathrm{R}$ \\
\hline ESO 328-36 & Sy1 & 0.0237 & 228.6958 & -40.35861 & 2200.9 & 384.8 & & \\
\hline IGR J15161-3827 & Sy2 & 0.0365 & 229.0375 & -38.44806 & 2181.2 & 372.1 & & \\
\hline NGC 5995 & Sy2 & 0.0252 & 237.1040 & -13.75778 & 1129.2 & 28.6 & & \\
\hline IGR J15539-6142 & Sy2 & 0.015 & 238.3967 & -61.68206 & 1754.0 & 101.0 & & \\
\hline IGR J16024-6107 & Sy2 & 0.0114 & 240.4517 & -61.14822 & 1792.4 & 84.3 & & \\
\hline IGR J16056-6110 & Sy1.5 & 0.052 & 241.4643 & -61.19525 & 1727.2 & 64.4 & & \\
\hline IGR J16119-6036 & Sy1 & 0.016 & 242.9642 & -60.63194 & 1789.6 & 60.9 & & \\
\hline IGR J16185-5928 & NLS1 & 0.035 & 244.6518 & -59.45482 & 2066.0 & 44.6 & $7.4 \pm 0.5^{p}$ & LL \\
\hline IGR J16351-5806 & Sy2 & 0.009 & 248.8071 & -58.08047 & 2230.3 & 39.3 & & \\
\hline IGR J16385-2057 & NLS1 & 0.0269 & 249.6250 & -20.94389 & 1505.9 & 80.4 & & \\
\hline IGR J16426+6536 & NLS1 & 0.323 & 250.7670 & 65.54747 & 56.0 & - & $7.0 \pm 0.5^{c}$ & $\mathrm{LL} / \mathrm{CL}$ \\
\hline IGR J16482-3036 & Sy1 & 0.0313 & 252.0623 & -30.58502 & 2450.9 & 53.7 & & \\
\hline ESO 138-1 & Sy2 & 0.0091 & 252.8333 & -59.23389 & 1509.2 & 21.4 & & \\
\hline NGC 6221 & Sy2 & 0.0050 & 253.1942 & -59.21639 & 1481.0 & 21.4 & & \\
\hline NGC 6240 & Sy2 & 0.0245 & 253.2457 & 2.40047 & 300.5 & 67.7 & & \\
\hline Mrk 501 & BLLac & 0.0337 & 253.4676 & 39.76017 & 476.3 & 70.6 & $9.2 \pm 0.3^{e}$ & $\mathrm{~S}$ \\
\hline IGR J16558-5203 & Sy1 & 0.054 & 254.0234 & -52.06135 & 3065.7 & 249.6 & $7.9 \pm 0.5^{p}$ & LL \\
\hline IGR J16562-3301 & BLLac & - & 254.0701 & -33.03680 & 3669.2 & 111.4 & & \\
\hline NGC 6300 & Sy2 & 0.0037 & 259.2467 & -62.81972 & 237.9 & 8.9 & $5.5 \pm 0.4^{c}$ & $X$ \\
\hline IGR J17204-3554 & AGN & - & 260.1042 & -35.90000 & 6318.5 & 292.3 & & \\
\hline QSO B1730-130 & QSO & 0.9020 & 263.2613 & -13.08042 & 1080.1 & 33.1 & & \\
\hline GRS 1734-292 & Sy1 & 0.0214 & 264.3681 & -29.13403 & 9122.1 & 665.7 & $8.9 \pm 0.7^{q}$ & SO \\
\hline IGR J17418-1212 & Sy1 & 0.0372 & 265.4625 & -12.19611 & 1300.1 & 45.5 & & \\
\hline IGR J17488-3253 & Sy1 & 0.020 & 267.2297 & -32.91449 & 6159.4 & 410.4 & & \\
\hline IGR J17513-2011 & Sy2 & 0.047 & 267.8068 & -20.20405 & 6220.1 & 268.7 & $6.0 \pm 0.5^{p}$ & LL \\
\hline IGR J18027-1455 & Sy1 & 0.0034 & 270.6974 & -14.91522 & 2463.8 & 173.0 & & \\
\hline IGR J18244-5622 & Sy2 & 0.0169 & 276.0812 & -56.36909 & 103.3 & 4.4 & & \\
\hline IGR J18249-3243 & Sy1 & 0.355 & 276.2361 & -32.71661 & 5126.8 & 208.7 & & \\
\hline IGR J18259-0706 & Sy1? & - & 276.48958 & -7.17264 & 2009.5 & 226.1 & & \\
\hline PKS 1830-211 & BLLac & 2.5070 & 278.4162 & -21.06106 & 2775.4 & 111.2 & & \\
\hline $3 \mathrm{C} 382$ & Sy1 & 0.0579 & 278.7641 & 32.69635 & 15.2 & 0.7 & $9.2 \pm 0.5^{n}$ & KM \\
\hline ESO 103-35 & Sy2 & 0.0133 & 279.5846 & -65.42805 & 28.5 & - & $7.1 \pm 0.6^{c}$ & $\mathrm{X}$ \\
\hline $3 \mathrm{C} 390.3$ & Sy1 & 0.0561 & 280.5374 & 79.77142 & 448.0 & 33.2 & $8.46_{-0.1}^{+0.09 g}$ & $\mathrm{R}$ \\
\hline ESO 140-43* & Sy1 & 0.0141 & 281.2917 & -62.35583 & 378.0 & 27.7 & & \\
\hline IGR J18559+1535 & Sy1 & 0.084 & 284.0000 & 15.63694 & 2594.9 & 24.7 & & \\
\hline ESO $141-55^{*}$ & Sy1 & 0.0366 & 290.3092 & -58.67083 & 378.0 & 79.9 & $7.1 \pm 0.6^{c}$ & SO \\
\hline 1RXS J192450.8-29143 & BLLac & 0.3520 & 291.2127 & -29.24170 & 955.7 & 28.6 & & \\
\hline 1H 1934-063 & Sy1 & 0.0106 & 294.3879 & -6.21806 & 654.2 & 2.1 & $7.9 \pm 0.6^{c}$ & SO \\
\hline IGR J19405-3016 & Sy1 & 0.052 & 295.0631 & -30.26347 & 930.3 & 22.3 & & \\
\hline NGC 6814 & Sy1.5 & 0.0052 & 295.6683 & -10.32333 & 438.5 & 7.4 & $7.1 \pm 0.2^{h}$ & $\mathrm{CL}$ \\
\hline IGR J19473+4452 & Sy2 & 0.0539 & 296.8307 & 44.82845 & 1194.3 & 1.0 & & \\
\hline $3 \mathrm{C} 403$ & Sy2 & 0.0590 & 298.0617 & 2.50778 & 490.2 & - & & \\
\hline QSO B1957+405 & Sy2 & 0.0561 & 299.8682 & 40.73386 & 2258.1 & 69.5 & $9.4 \pm 0.1^{l}$ & $\mathrm{~K}$ \\
\hline 1ES $1959+650^{x}$ & BLLac & 0.048 & 299.9994 & 65.14851 & 11.2 & - & $8.1 \pm 0.3^{e}$ & $\mathrm{~S}$ \\
\hline ESO 399-20 & NLS1 & 0.0250 & 301.7383 & -34.54833 & 857.2 & 24.7 & & \\
\hline IGR J20187+4041 & Sy2 & $0.0144^{r}$ & 304.6606 & 40.68344 & 2851.0 & 442.7 & & \\
\hline IGR J20286+2544 & Sy2 & 0.013 & 307.1462 & 25.73361 & 840.2 & 4.1 & & \\
\hline $4 \mathrm{C} 74.26^{x}$ & QSO & 0.1040 & 310.6549 & 75.13403 & 77.1 & - & $9.6 \pm 0.5^{e}$ & CL \\
\hline Mrk 509 & Sy 1.2 & 0.0344 & 311.0406 & -10.72348 & 71.7 & 57.3 & $8.16_{-0.04}^{+0.03 g}$ & $\mathrm{R}$ \\
\hline S5 $2116+81$ & Sy1 & 0.086 & 318.5021 & 82.07975 & 176.5 & - & $8.8 \pm 0.5^{-0.04}$ & KM \\
\hline IGR J21178+5139 & AGN & - & 319.4468 & 51.64823 & 1261.9 & 171.3 & & \\
\hline IGR J21247+5058 & Sy1 & 0.020 & 321.1640 & 50.97329 & 1392.7 & 174.7 & & \\
\hline IGR J21272+4241 ${ }^{x}$ & Sy1.5 & 0.316 & 321.7917 & 42.69194 & 836.3 & 189.8 & & \\
\hline IGR J21277+5656 & Sy1 & 0.0144 & 321.9373 & 56.94436 & 984.5 & 166.6 & & \\
\hline RX J2135.9+4728 & Sy1 & 0.0252 & 323.9766 & 47.47453 & 1213.4 & 136.3 & & \\
\hline PKS 2149-306 & FSRQ & 2.345 & 327.9813 & -30.46492 & 258.6 & 47.1 & & \\
\hline NGC 7172 & Sy2 & 0.0086 & 330.5071 & -31.87167 & 346.5 & 68.8 & $7.7 \pm 0.6^{c}$ & SO \\
\hline BL Lac & BLLac & 0.0686 & 330.6804 & 42.27778 & 738.1 & - & $8.2 \pm 0.7^{e}$ & SB \\
\hline IGR J22292+6647 & $\mathrm{Sy} 1^{o}$ & $0.113^{\circ}$ & 337.3062 & 66.78106 & 1678.9 & 9.3 & & \\
\hline NGC 7314 & Sy1 & 0.0048 & 338.9419 & -26.05047 & 546.8 & 1.7 & $6.0 \pm 0.5^{c}$ & $\mathrm{~S}$ \\
\hline Mrk 915 & Sy1 & 0.0241 & 339.1938 & -12.54517 & 609.0 & 12.8 & & \\
\hline IGR J22517+2217 & BLLac & 3.668 & 342.9280 & 22.29900 & 251.3 & 5.8 & & \\
\hline $3 \mathrm{C} 454.3$ & BLLac & 0.8590 & 343.4906 & 16.14822 & 207.1 & 36.3 & $9.2 \pm 0.7^{e}$ & CL \\
\hline
\end{tabular}


Table 1. continued.

\begin{tabular}{lccrrrrrr}
\hline \hline Name & Type & $z$ & $\begin{array}{r}\text { RA } \\
{[\mathrm{deg}]}\end{array}$ & $\begin{array}{r}\text { Dec } \\
{[\mathrm{deg}]}\end{array}$ & $\begin{array}{r}\text { ISGRI } \\
{[\mathrm{ks}]}\end{array}$ & $\begin{array}{r}\text { JEM-X } \\
{[\mathrm{ks}]}\end{array}$ & $\begin{array}{r}\log M_{\mathrm{BH}} \\
{\left[M_{\odot}\right]}\end{array}$ & Method \\
\hline 1H 2251-179 & Sy1 & 0.0640 & 343.5245 & -17.58203 & 579.2 & 314.6 & $<6.9^{c}$ & $\mathrm{~W}$ \\
NGC 7469 & Sy1 & 0.0163 & 345.8156 & 8.87386 & 139.2 & - & $7.09 \pm 0.05^{g}$ & $\mathrm{R}$ \\
MCG-02-58-022 & Sy1.5 & 0.0469 & 346.1812 & -8.68572 & 624.5 & 28.2 & $7.1 \pm 0.6^{c}$ & SO \\
NGC 7603 & Sy1.5 & 0.0295 & 349.7359 & 0.24347 & 104.5 & - & $8.1 \pm 0.3^{e}$ & $\mathrm{~S}$ \\
IGR J23206+6431 & Sy1 & 0.0732 & 350.15 & 64.52 & 3888.2 & 349.8 & & \\
IGR J23308+7120 & Sy2 & 0.037 & 352.6552 & 71.37911 & 1965.1 & - & & \\
IGR J23524+5842 & Sy2 & 0.164 & 358.0917 & 58.75908 & 4076.7 & 361.1 & & \\
\hline
\end{tabular}

${ }^{*}$ Beckmann et al. (2007b); ${ }^{a}$ Masetti et al. (2009); ${ }^{b}$ Bian \& Gu (2007); ${ }^{c}$ Middleton et al. (2008); ${ }^{d}$ Kaspi et al. (2000); ${ }^{e}$ Woo \& Urry (2002); ${ }^{f}$ Merloni et al. (2003); ${ }^{g}$ Peterson et al. (2004); ${ }^{h}$ Hicks \& Malkan (2008); ${ }^{i}$ Uttley \& McHardy (2005); ${ }^{k}$ Paltani \& Türler (2005); ${ }^{l}$ Graham (2008); ${ }^{m}$ Zurita-Heras et al. (2009); ${ }^{n}$ Winter et al. (2009); ${ }^{o}$ Butler et al. (2009); ${ }^{p}$ Masetti et al. (2006); ${ }^{q}$ see Appendix A; ${ }^{r}$ Goncalves et al. (2009); ${ }^{x}$ not detected in the data set presented here.

the central black hole $M_{\mathrm{BH}}$ in an AGN or a normal galaxy, most of them still carrying fairly large uncertainties. Nevertheless, considering the importance of the black hole mass in studying the properties of these objects, we decided to include a compilation of the mass estimates from the literature as the best guess that can be provided at present for each object in this catalogue.

We have included masses estimated from gas and/or stellar kinematics in the nuclear region of the galaxy, in the presence (method "M", see e.g. Greenhill 1997) or not ("K", Hicks \& Malkan 2008) of a water maser, from assuming virialized motions of the broad line region (BLR) clouds, either using the reverberation-mapping technique ("R", Kaspi et al. 2000) or estimating the size of the BLR from the emission line luminosity (of the $\mathrm{H} \beta$ line usually; "LL", Wu et al. 2004) or from the optical continuum luminosity (usually measured at $5100 \AA$; "CL", Kaspi et al. 2000). Other methods are based on the empirical relation between the black hole mass and the stellar velocity dispersion $\sigma_{\mathrm{s}}$, using either direct measurements of the latter ("S", Ferrarese \& Merritt 2000) or indirect estimates of $\sigma_{\mathrm{s}}$ from the width of the [O III] line ("SO", Greene \& Ho 2005) or from the morphological parameters of the bulge ("SB", O'Dowd et al. 2002). Some estimates use the bulge luminosity ("B", Wandel 2002), the $K$-band stellar magnitude (assuming that it is dominated by the bulge; "KM", Novak et al. 2006), the X-ray variability time scales ("X", Gierliński et al. 2008), or the properties of outflowing warm absorber clouds ("W", Morales \& Fabian 2002). Whenever the uncertainty on the estimate of the black hole mass is not available in the reference paper, we assumed a conservative one following the typical uncertainties of the method used for the mass measurement.

The most reliable methods are those involving direct measurements of gas and stellar kinematics, with average uncertainties in the range $0.15-0.3 \mathrm{dex}$, reaching $0.1 \mathrm{dex}$ or less when water maser emission is detected (see Vestergaard 2004, for more details). Also the reverberation mapping technique provides black hole masses with accuracy around 0.15-0.3 dex, which drops to values of $0.4-0.5 \mathrm{dex}$ and even to $1 \mathrm{dex}$ when the radius of the BLR is estimated from the emission line or the continuum luminosity. Masses estimated from the stellar velocity dispersion can have uncertainties around $0.3 \operatorname{dex}$ when $\sigma_{\mathrm{s}}$ is directly measured, while indirect measurements of $\sigma_{\mathrm{s}}$ result in much less precise estimates $(\geq 0.7 \mathrm{dex})$. Larger uncertainties are provided by the other methods mentioned above, $0.5-0.6 \mathrm{dex}$ for method "B", 0.5-1 dex for "X" (Awaki et al. 2005), 0.5 dex for "KM" (Winter et al. 2009), and only upper limits can be derived with the method based on outflowing warm absorber clouds.

\subsection{X-ray spectral fitting}

For all 187 objects with a detection significance above $3 \sigma$ in the IBIS/ISGRI 18-60 keV energy band, spectral analysis was performed using an absorbed power law with $N_{\mathrm{H}}$ fixed to the value reported in the literature (Table 2) and adopting XSPEC version 11.3.2 (Arnaud 1996). When the significance was below $5 \sigma$, the photon index was fixed to $\Gamma=2$. The $N_{\mathrm{H}}$ value used for the fitting is the intrinsic absorption plus the Galactic hydrogen column density, whereas in Table 2 only the intrinsic absorption is reported. In cases where no absorption information was found, Swift/XRT and XMM-Newton data were analysed to determine the level of absorption. For those objects detected only at a low significance level (i.e. between 3 and $5 \sigma$ ), the photon index was fixed to $\Gamma=2.0$ in order to extract a flux value. Table 2 gives the fit results to the IBIS/ISGRI data. Fluxes are model fluxes according to the best-fit result. In the cases where a cut-off power law model gave a significantly better fit to the ISGRI data we set the $\Gamma$ column to " $C$ ". For these 12 objects, the fluxes reported are based on the best-fit model reported in Table 3. These 12 sources and all sources that gave a high IBIS/ISGRI detection significance of $>30 \sigma$ are also discussed in more detail in Appendix A.

The JEM-X spectra of the 23 AGN detected by the X-ray monitor were fit with the IBIS/ISGRI data and results reported in Table 4. As for the ISGRI spectra alone, we also did not fit the absorption values in the case of the combined JEM-X/ISGRI spectra, because the JEM-X data starting at $3 \mathrm{keV}$ did not allow a significant constraint on $N_{\mathrm{H}}$ in most cases. In cases where the flux of the source varied significantly, so no combined fit could be performed resulting in $\chi_{v}^{2}<2$, only simultaneous data were used (e.g. in the case of NGC 4388). For two AGN, NGC 1275 and IGR J17488-3253, a more complex model than an absorbed cut-off power law was required to represent the combined JEM-X and IBIS/ISGRI data (see Appendix A).

\subsection{Optical data}

Optical data in the $V$ band are provided by the optical monitoring camera (OMC). Data were extracted from the OMC Archive ${ }^{2}$ getting one photometric point per shot. The photometric apertures were centred on the source position, as listed in version 5 of the OMC Input Catalogue (Domingo et al. 2003). The fluxes and magnitudes were derived from a photometric aperture of $3 \times 3$ pixels $(1$ pixel $=17.504$ arcsec $)$, slightly circularized,

\footnotetext{
${ }^{2}$ http://sdc.laeff.inta.es/omc/
} 
Table 2. Spectral fit results for IBIS/ISGRI data.

\begin{tabular}{|c|c|c|c|c|c|c|}
\hline Name & $\begin{array}{r}\text { ISGRI } \\
18-60 \mathrm{keV}[\sigma]\end{array}$ & $\begin{array}{c}N_{\mathrm{H}} \\
{\left[10^{22} \mathrm{~cm}^{-2}\right]}\end{array}$ & $\begin{array}{c}f_{20-40 \mathrm{keV}} \\
{\left[10^{-11} \mathrm{erg} \mathrm{cm}^{-2} \mathrm{~s}^{-1}\right]}\end{array}$ & $\begin{array}{c}f_{40-100 \mathrm{keV}} \\
{\left[10^{-11} \mathrm{erg} \mathrm{cm}^{-2} \mathrm{~s}^{-1}\right]}\end{array}$ & $\overline{\Gamma_{\text {ISGRI }}^{+}}$ & $\begin{array}{c}\log L_{20-100 \mathrm{keV}} \\
{\left[\mathrm{erg} \mathrm{s}^{-1}\right]}\end{array}$ \\
\hline IGR J00254+6822 & 7.5 & $40^{c}$ & 0.6 & 0.8 & $2.1 \pm 0.5$ & 42.67 \\
\hline IGR J00335+6126 & 5.9 & $0.5^{*}$ & 0.3 & 0.9 & $1.1 \pm 0.2$ & 44.50 \\
\hline 1ES $0033+59.5$ & 12.6 & $0.36^{a}$ & 0.8 & 0.3 & $3.6_{-0.3}^{+0.4}$ & 44.36 \\
\hline Mrk 348 & 14.6 & $30^{b}$ & 4.2 & 6.6 & $\mathrm{C}^{-0.3}$ & 43.74 \\
\hline NGC 526A & 4.3 & $1.6^{b}$ & 2.2 & 2.9 & 2 & 43.62 \\
\hline ESO 297-18 & 5.2 & $42^{i}$ & 3.1 & 6.8 & $1.5 \pm 0.3$ & 44.10 \\
\hline IGR J01528-0326 & 8.5 & $14^{c}$ & 0.9 & 2.3 & $1.2_{-0.5}^{+0.2}$ & 43.30 \\
\hline NGC 788 & 23.4 & $<0.02^{a}$ & 2.5 & 3.9 & $1.8 \pm 0.1$ & 43.42 \\
\hline Mrk 590 & 4.5 & 0.03 & 0.5 & 0.6 & 2 & 43.23 \\
\hline IGR J02097+5222 & 7.7 & $0.03^{i}$ & 1.2 & 2.0 & $1.7_{-0.2}^{+0.3}$ & 44.25 \\
\hline SWIFT J0216.3+5128 & 5.1 & $1.27^{f}$ & 0.8 & 0.8 & $2.3_{-1.0}^{+1.2}$ & 43.48 \\
\hline Mrk 1040 & 3.4 & $0.067^{b}$ & 2.3 & 3.0 & $2^{-1.0}$ & 43.52 \\
\hline IGR J02343+3229 & 3.5 & $2.2^{d}$ & 1.4 & 1.8 & 2 & 43.27 \\
\hline NGC 985 & 5.8 & $0.6^{b}$ & 0.9 & 1.3 & $2.0_{-0.3}^{+0.4}$ & 43.99 \\
\hline NGC 1052 & 5.6 & $0.041^{b}$ & 0.8 & 1.5 & $1.6_{-0.4}^{+0.8}$ & 42.11 \\
\hline RBS 345 & 5.0 & - & 0.9 & 1.9 & $1.3_{-0.4}^{+0.6}$ & 44.49 \\
\hline NGC 1068 & 7.3 & $>150^{a}$ & 1.2 & 1.3 & $2.3_{-0.3}^{+0.4}$ & 43.68 \\
\hline QSO B0241+62 & 16.3 & $1.5^{a}$ & 2.0 & 3.0 & $1.8 \pm 0.1$ & 44.37 \\
\hline IGR J02466-4222 & 3.3 & 1 & 1.0 & 1.4 & 2 & 44.45 \\
\hline IGR J02501+5440 & 5.1 & - & 0.6 & 1.5 & $1.2 \pm 0.3$ & 43.03 \\
\hline MCG-02-08-014 & 6.3 & - & 1.3 & 0.9 & $2.7_{-0.5}^{+0.6}$ & 43.15 \\
\hline NGC 1142 & 16.2 & $45^{b}$ & 2.7 & 4.2 & $1.8 \pm 0.1$ & 44.11 \\
\hline QSO B0309+411 & 4.0 & $<0.1^{*}$ & 3.0 & 3.9 & 2 & 45.53 \\
\hline NGC 1275 & 15.7 & $3.75^{a}$ & 2.2 & 0.4 & $3.7_{-0 .}^{+0.3}$ & 43.18 \\
\hline $1 \mathrm{H} 0323+342$ & 5.1 & $0.1^{b}$ & 0.7 & 2.4 & $1.0_{-0.7}^{+0.2}$ & 44.43 \\
\hline IGR J03334+3718 & 6.9 & - & 1.0 & 1.5 & $1.9 \pm 0.3$ & 44.25 \\
\hline NGC 1365 & 5.1 & $44^{b}$ & 1.5 & 2.2 & $1.8 \pm 0.6$ & 42.38 \\
\hline IGR J03532-6829 & 7.3 & $0.05^{*}$ & 1.4 & 0.6 & $3.5_{-0.6}^{+0.7}$ & 44.63 \\
\hline $3 \mathrm{C} 111$ & 13.7 & $0.63^{a}$ & 5.3 & 6.9 & $2.0 \pm 0.2$ & 44.83 \\
\hline $3 \mathrm{C} 120$ & 22.0 & $0.2^{i}$ & 3.0 & 4.4 & $1.8 \pm 0.1$ & 44.27 \\
\hline UGC 3142 & 17.3 & $1.4^{x}$ & 2.8 & 3.7 & $2.0 \pm 0.1$ & 44.84 \\
\hline LEDA 168563 & 5.4 & $<0.22^{i}$ & 2.2 & 3.1 & $1.9_{-0.4}^{+0.5}$ & 44.00 \\
\hline ESO 33-2 & 15.5 & $0.1^{b}$ & 1.3 & 2.0 & $1.8^{-0.4} \pm 0.2$ & 43.38 \\
\hline $4 \mathrm{U} 0517+17$ & 24.5 & $0.1^{b}$ & 3.0 & 3.6 & $2.13_{-0.08}^{+0.09}$ & 43.68 \\
\hline Ark 120 & 15.6 & $<0.1^{*}$ & 2.7 & 3.4 & $2.1 \pm 0.1$ & 44.18 \\
\hline NGC 2110 & 4.9 & $4.3^{*}$ & 7.0 & 9.3 & 2 & 43.34 \\
\hline MCG+08-11-011 & 7.8 & $0.183^{b}$ & 5.2 & 6.7 & $2.0 \pm 0.4$ & 44.05 \\
\hline IRAS 05589+2828 & 15.4 & $<0.04^{i}$ & 1.4 & 2.8 & $1.5_{-0.1}^{+0.2}$ & 44.02 \\
\hline SWIFT J0601.9-8636 & 6.5 & $5.6^{i}$ & 1.3 & 1.0 & $2.6_{-0.6}^{+0.1}$ & 42.32 \\
\hline IGR J06117-6625 & 17.8 & $0.048^{b}$ & 1.9 & 2.6 & $1.96 \pm 0.16$ & 45.85 \\
\hline Mrk 3 & 26.7 & $110^{a}$ & 3.7 & 6.4 & $1.73 \pm 0.07$ & 43.61 \\
\hline IGR J06239-6052 & 3.6 & $20^{h}$ & 0.5 & 0.7 & 2 & 43.65 \\
\hline PKS 0637-752 & 5.4 & $0.035^{b}$ & 0.7 & 1.1 & $1.7 \pm 0.6$ & 46.45 \\
\hline Mrk 6 & 15.1 & $10^{a}$ & 1.9 & 2.3 & $2.1 \pm 0.2$ & 43.53 \\
\hline QSO B0716+714 & 4.6 & $<0.01^{a}$ & 0.3 & 0.3 & 2 & 45.24 \\
\hline LEDA 96373 & 5.3 & - & 1.4 & 2.6 & $1.5_{-0.5}^{+0.7}$ & 43.89 \\
\hline IGR J07437-5137 & 3.5 & - & 0.4 & 0.5 & $2^{-0.5}$ & 43.13 \\
\hline IGR J07565-4139 & 6.2 & $1.1^{b}$ & 0.7 & 0.6 & $2.7_{-0.4}^{+0.6}$ & 43.12 \\
\hline IGR J07597-3842 & 14.2 & $0.05^{b}$ & 1.8 & 1.9 & $2.3 \pm 0.2$ & 44.14 \\
\hline ESO 209-12 & 12.2 & $0.1^{b}$ & 0.9 & 1.4 & $1.8_{-0.2}^{+0.1}$ & 43.93 \\
\hline PG 0804+761 & 6.3 & $0.023^{b}$ & 1.1 & 1.5 & $2.0 \pm 0.5$ & 44.81 \\
\hline Fairall 1146 & 9.9 & $0.1^{b}$ & 0.9 & 1.4 & $1.8_{-0.2}^{+0.3}$ & 43.73 \\
\hline QSO B0836+710 & 15.4 & $0.11^{a}$ & 2.1 & 4.1 & $1.5_{-0.1}^{+0.2}$ & 47.93 \\
\hline IGR J09026-4812 & 17.8 & $0.9^{w}$ & 0.9 & 1.4 & $1.9 \pm 0.1$ & 43.91 \\
\hline SWIFT J0917.2-6221 & 5.8 & $0.5^{f}$ & 0.8 & 1.1 & $1.9_{-0.6}^{+0.5}$ & 44.18 \\
\hline IGR J09253+6929 & 7.0 & $8^{d}$ & 1.8 & 2.6 & $1.9 \pm 0.4$ & 44.19 \\
\hline Mrk 110 & 3.6 & $0.019^{b}$ & 2.2 & 3.0 & 2 & 44.18 \\
\hline IGR J09446-2636 & 3.2 & $0.1^{b}$ & 1.3 & 1.7 & 2 & 45.60 \\
\hline
\end{tabular}


Table 2. continued.

\begin{tabular}{|c|c|c|c|c|c|c|}
\hline Name & $\begin{array}{r}\text { ISGRI } \\
18-60 \mathrm{keV}[\sigma]\end{array}$ & $\begin{array}{c}N_{\mathrm{H}} \\
{\left[10^{22} \mathrm{~cm}^{-2}\right]}\end{array}$ & $\begin{array}{c}f_{20-40 \mathrm{keV}} \\
{\left[10^{-11} \mathrm{erg} \mathrm{cm}^{-2} \mathrm{~s}^{-1}\right]}\end{array}$ & $\begin{array}{c}f_{40-100 \mathrm{keV}} \\
{\left[10^{-11} \mathrm{erg} \mathrm{cm}^{-2} \mathrm{~s}^{-1}\right]}\end{array}$ & $\Gamma_{\text {ISGRI }}^{+}$ & $\begin{array}{c}\log L_{20-100 \mathrm{keV}} \\
{\left[\mathrm{erg} \mathrm{s}^{-1}\right]}\end{array}$ \\
\hline NGC 2992 & 15.8 & $0.1^{l}$ & 2.8 & 4.1 & $1.9 \pm 0.1$ & 42.95 \\
\hline MCG-05-23-016 & 14.6 & $1.6^{a}$ & 5.6 & 8.1 & $1.9 \pm 0.1$ & 43.34 \\
\hline IGR J09523-6231 & 5.3 & $8^{d}$ & 0.6 & 0.7 & $2.2_{-0.5}^{+0.6}$ & 45.41 \\
\hline NGC 3081 & 9.7 & $66^{m}$ & 2.6 & 4.1 & $1.8 \pm 0.2$ & 42.97 \\
\hline SWIFT J1009.3-4250 & 6.9 & $30^{f}$ & 1.5 & 2.0 & $2.0 \pm 0.4$ & 43.95 \\
\hline IGR J10147-6354 & 3.0 & $2^{d}$ & 0.5 & 0.7 & 2 & 45.18 \\
\hline NGC 3227 & 16.6 & $6.8^{b}$ & 5.6 & 7.1 & $2.0 \pm 0.1$ & 42.63 \\
\hline NGC 3281 & 7.5 & $151^{b}$ & 1.8 & 2.7 & $1.9 \pm 0.3$ & 43.06 \\
\hline SWIFT J1038.8-4942 & 5.3 & $0.6^{f}$ & 0.6 & 1.4 & $1.3 \pm 0.4$ & 44.21 \\
\hline IGR J10404-4625 & 6.9 & $3^{f}$ & 1.2 & 1.4 & $2.2_{-0.3}^{+0.4}$ & 43.54 \\
\hline Mrk 421 & 173.8 & $0.08^{i}$ & 22.4 & 20.6 & $2.45_{-0.02}^{0.03}$ & 44.92 \\
\hline IGR J11366-6002 & 7.6 & $0.35^{\circ}$ & 0.6 & 0.7 & $2.2_{-0.4}^{+0.52}$ & 42.73 \\
\hline NGC 3783 & 6.1 & $0.08^{*}$ & 6.0 & 8.4 & $1.9 \pm 0.4$ & 43.48 \\
\hline IGR J12026-5349 & 14.8 & $2.2^{a}$ & 1.6 & 2.2 & $2.0 \pm 0.1$ & 43.84 \\
\hline NGC 4051 & 11.7 & $<0.02^{*}$ & 1.8 & 1.5 & $2.1 \pm 0.2$ & 41.58 \\
\hline NGC 4138 & 9.0 & $8^{b}$ & 1.2 & 1.9 & $1.8 \pm 0.3$ & 41.79 \\
\hline NGC 4151 & 205.7 & $6.9^{a}$ & 24.0 & 32.3 & $\mathrm{C}$ & 43.13 \\
\hline NGC 4180 & 9.4 & - & 0.9 & 1.8 & $1.6_{-0.2}^{+0.3}$ & 42.46 \\
\hline Was 49 & 3.8 & $10^{b}$ & 0.6 & 0.8 & $2^{-0.2}$ & 44.12 \\
\hline Mrk 766 & 6.9 & $0.8^{a}$ & 1.0 & 1.5 & $1.8 \pm 0.3$ & 42.95 \\
\hline NGC 4258 & 6.6 & $8.7^{b}$ & 1.1 & 1.1 & $2.3 \pm 0.4$ & 41.04 \\
\hline 4C 04.42 & 7.7 & $0.1^{b}$ & 0.7 & 1.8 & $1.2 \pm 0.2$ & 46.83 \\
\hline Mrk 50 & 6.2 & $0.018^{b}$ & 0.5 & 0.4 & $2.6_{-0.6}^{+0.7}$ & 43.09 \\
\hline NGC 4388 & 78.1 & $27^{a}$ & 9.8 & 15.2 & $\mathrm{C}$ & 43.59 \\
\hline NGC 4395 & 8.8 & $0.15^{a}$ & 1.5 & 1.6 & $2.3_{-0.3}^{+0.4}$ & 40.85 \\
\hline $3 \mathrm{C} 273$ & 78.0 & $0.5^{a}$ & 7.5 & 10.6 & $1.92 \pm 0.03$ & 46.09 \\
\hline NGC 4507 & 30.9 & $29^{a}$ & 6.3 & 10.0 & $\mathrm{C}$ & 43.70 \\
\hline SWIFT J1238.9-2720 & 6.3 & $60^{f}$ & 2.7 & 5.3 & $1.6_{-0.3}^{+0.4}$ & 44.06 \\
\hline IGR J12391-1612 & 9.9 & $3^{f}$ & 1.7 & 2.2 & $2.0 \pm 0.2$ & 44.08 \\
\hline NGC 4593 & 33.0 & $0.02^{a}$ & 3.2 & 4.1 & $\mathrm{C}$ & 43.08 \\
\hline IGR J12415-5750 & 6.7 & $<0.11^{b}$ & 0.8 & 1.4 & $1.7_{-0.3}^{+0.2}$ & 43.45 \\
\hline PKS 1241-399 & 3.5 & - & 0.7 & 0.9 & 2 & 45.23 \\
\hline ESO 323-32 & 5.6 & $7^{f}$ & 0.8 & 1.5 & $1.6_{-0.3}^{+0.4}$ & 43.13 \\
\hline 3C 279 & 8.6 & $\lessgtr 0.13^{a}$ & 0.9 & 1.7 & $1.6 \pm 0.2$ & 46.40 \\
\hline Mrk 783 & 4.7 & $0.046^{b}$ & 0.9 & 1.2 & 2 & 44.36 \\
\hline IGR J13038+5348 & 4.5 & $<0.03^{*}$ & 1.0 & 1.3 & 2 & 43.67 \\
\hline NGC 4945 & 78.7 & $400^{a}$ & 9.9 & 18.4 & $\mathrm{C}$ & 42.35 \\
\hline IGR J13057+2036 & 3.1 & - & 0.4 & 0.6 & 2 & \\
\hline ESO 323-77 & 9.0 & $55^{a}$ & 1.1 & 2.1 & $1.5_{-0.2}^{+0.3}$ & 43.21 \\
\hline IGR J13091+1137 & 7.5 & $90^{a}$ & 1.6 & 2.5 & $2.1 \pm 0.3$ & 43.76 \\
\hline IGR J13109-5552 & 8.7 & $<0.1^{p}$ & 0.9 & 1.2 & $2.0_{-0.2}^{+0.3}$ & 44.78 \\
\hline NGC 5033 & 4.2 & $0.03^{b}$ & 0.6 & 0.8 & 2 & 41.43 \\
\hline IGR J13149+4422 & 4.7 & $5^{d}$ & 0.8 & 1.1 & 2 & 43.77 \\
\hline Cen A & 247.2 & $12.5^{a}$ & 28.1 & 43.4 & $1.82 \pm 0.01$ & 42.71 \\
\hline ESO 383-18 & 6.5 & $17^{x}$ & 0.9 & 1.2 & $1.9_{-0.3}^{+0.4}$ & 42.86 \\
\hline MCG-06-30-015 & 21.1 & $0.03^{b}$ & 2.3 & 1.7 & $2.4 \pm 0.1$ & 42.74 \\
\hline NGC 5252 & 3.0 & $0.68^{b}$ & 2.0 & 2.7 & 2 & 43.76 \\
\hline Mrk 268 & 5.7 & - & 0.9 & 0.7 & $2.5 \pm 0.6$ & 43.78 \\
\hline 4U 1344-60 & 35.2 & $5^{a}$ & 3.5 & 4.6 & C & 43.48 \\
\hline IC 4329A & 58.5 & $0.42^{a}$ & 8.8 & 12.3 & $\mathrm{C}$ & 44.09 \\
\hline Circinus Galaxy & 120.0 & $360^{a}$ & 10.9 & 10.1 & C & 41.96 \\
\hline NGC 5506 & 27.8 & $3.4^{a}$ & 7.0 & 6.2 & $2.1 \pm 0.1$ & 43.16 \\
\hline IGR J14175-4641 & 8.0 & - & 0.8 & 1.3 & $1.8 \pm 0.3$ & 44.48 \\
\hline NGC 5548 & 3.1 & $0.51^{a}$ & 1.0 & 1.3 & 2 & 43.19 \\
\hline RHS 39 & 7.7 & $<0.05^{a}$ & 1.6 & 2.2 & $2.0 \pm 0.3$ & 43.63 \\
\hline H $1426+428$ & 4.6 & $<0.02^{*}$ & 0.6 & 0.8 & 2 & 44.80 \\
\hline IGR J14471-6414 & 7.2 & $<0.1^{*}$ & 0.5 & 0.8 & $1.8 \pm 0.3$ & 43.95 \\
\hline IGR J14471-6319 & 6.4 & $2^{f}$ & 0.6 & 0.8 & $2.0_{-0.6}^{+0.5}$ & 43.67 \\
\hline IGR J14492-5535 & 8.6 & $12^{q}$ & 0.9 & 1.4 & $1.7 \pm 0.3$ & \\
\hline
\end{tabular}


Table 2. continued.

\begin{tabular}{|c|c|c|c|c|c|c|}
\hline Name & $\begin{array}{r}\text { ISGRI } \\
18-60 \mathrm{keV}[\sigma]\end{array}$ & $\begin{array}{c}N_{\mathrm{H}} \\
{\left[10^{22} \mathrm{~cm}^{-2}\right]}\end{array}$ & $\begin{array}{c}f_{20-40 \mathrm{keV}} \\
{\left[10^{-11} \mathrm{erg} \mathrm{cm}^{-2} \mathrm{~s}^{-1}\right]}\end{array}$ & $\begin{array}{c}f_{40-100 \mathrm{keV}} \\
{\left[10^{-11} \mathrm{erg} \mathrm{cm}^{-2} \mathrm{~s}^{-1}\right]}\end{array}$ & $\overline{\Gamma_{\text {ISGRI }}^{+}}$ & $\begin{array}{c}\log L_{20-100 \mathrm{keV}} \\
{\left[\mathrm{erg} \mathrm{s}^{-1}\right]}\end{array}$ \\
\hline IGR J14515-5542 & 9.4 & $0.4^{f}$ & 0.8 & 1.4 & $1.6 \pm 0.2$ & 43.19 \\
\hline IGR J14552-5133 & 8.5 & $0.1^{b}$ & 0.6 & 1.1 & $1.6_{-0.2}^{+0.4}$ & 42.98 \\
\hline IGR J14561-3738 & 9.4 & $>100^{q}$ & 0.6 & 1.2 & $1.7_{-0.4}^{-0.2}$ & 43.37 \\
\hline IGR J14579-4308 & 15.4 & $20^{d}$ & 1.1 & 1.4 & $2.0 \pm 0.2$ & 43.16 \\
\hline Mrk 841 & 3.2 & 0.21 & 1.0 & 1.4 & 2 & 43.87 \\
\hline ESO 328-36 & 5.7 & - & 0.4 & 0.4 & $2.5 \pm 0.6$ & 43.00 \\
\hline IGR J15161-3827 & 12.3 & - & 1.0 & 1.7 & $1.7 \pm 0.2$ & 43.90 \\
\hline NGC 5995 & 16.4 & $0.7^{*}$ & 2.2 & 1.5 & $2.8 \pm 0.2$ & 43.74 \\
\hline IGR J15539-6142 & 4.6 & $18^{f}$ & 0.5 & 0.7 & 2 & 42.78 \\
\hline IGR J16024-6107 & 3.7 & $<0.1^{o}$ & 0.3 & 0.4 & 2 & 42.46 \\
\hline IGR J16056-6110 & 3.8 & $<1^{c}$ & 0.3 & 0.5 & 2 & 43.72 \\
\hline IGR J16119-6036 & 9.3 & $0.1^{b}$ & 0.9 & 1.4 & $1.8_{-0.2}^{+0.3}$ & 43.11 \\
\hline IGR J16185-5928 & 6.1 & $<0.1^{e}$ & 0.5 & 0.9 & $1.6 \pm 0.3$ & 43.57 \\
\hline IGR J16351-5806 & 10.2 & $<0.1^{s}$ & 0.8 & 1.6 & $1.5 \pm 0.2$ & 42.64 \\
\hline IGR J16385-2057 & 7.2 & $0.21^{d}$ & 0.8 & 0.5 & $3.1 \pm 0.4$ & 43.35 \\
\hline IGR J16426+6536 & 4.4 & - & 2.6 & 3.4 & 2 & 46.32 \\
\hline IGR J16482-3036 & 27.5 & $0.13^{f}$ & 2.5 & 3.1 & $2.1 \pm 0.1$ & 44.10 \\
\hline ESO 138-1 & 3.7 & $150^{b}$ & 0.4 & 0.6 & 2 & 42.26 \\
\hline NGC 6221 & 4.7 & $1^{a}$ & 0.5 & 1.0 & 2 & 41.93 \\
\hline NGC 6240 & 12.1 & $2^{b}$ & 2.1 & 3.6 & $1.7 \pm 0.2$ & 43.89 \\
\hline Mrk 501 & 12.4 & $0.013^{b}$ & 2.5 & 1.7 & $2.8 \pm 0.3$ & 44.05 \\
\hline IGR J16558-5203 & 16.5 & $0.011^{b}$ & 1.1 & 1.5 & $2.0 \pm 0.1$ & 44.25 \\
\hline IGR J16562-3301 & 7.0 & $0.2^{f}$ & 1.0 & 2.3 & $1.32_{-0.07}^{+0.16}$ & \\
\hline NGC 6300 & 10.1 & $22^{a}$ & 3.1 & 3.6 & $2.2 \pm 0.2$ & 42.30 \\
\hline IGR J17204-3554 & 6.4 & $12^{b}$ & 0.3 & 0.4 & $2.2 \pm 0.5$ & \\
\hline GRS 1734-292 & 106.4 & $3.7^{a}$ & 4.3 & 4.4 & $\mathrm{C}$ & 43.96 \\
\hline IGR J17418-1212 & 6.6 & $0.1^{b}$ & 0.8 & 1.5 & $1.5 \pm 0.2$ & 43.85 \\
\hline IGR J17488-3253 & 27.5 & $0.7^{*}$ & 1.1 & 1.6 & $\mathrm{C}$ & 43.39 \\
\hline IGR J17513-2011 & 16.0 & $0.6^{*}$ & 0.8 & 1.1 & $1.95 \pm 0.12$ & 44.00 \\
\hline IGR J18027-1455 & 15.2 & $19.0^{a}$ & 1.2 & 1.7 & $1.9 \pm 0.1$ & 41.86 \\
\hline IGR J18244-5622 & 3.0 & $14^{f}$ & 1.3 & 1.7 & 2 & 43.28 \\
\hline IGR J18249-3243 & 7.5 & $<0.1^{n}$ & 0.5 & 0.7 & $1.9 \pm 0.4$ & 45.72 \\
\hline IGR J18259-0706 & 7.8 & $0.6^{f}$ & 0.6 & 0.8 & $2.1 \pm 0.3$ & \\
\hline PKS 1830-211 & 25.2 & $\lesssim 0.7^{a}$ & 1.9 & 3.7 & $1.49_{-0.07}^{+0.05}$ & 48.19 \\
\hline $3 \mathrm{C} 382$ & 3.0 & $0.88^{b}$ & 3.8 & 5.0 & $2^{-0.0 r}$ & 44.85 \\
\hline ESO 103-35 & 4.9 & $19^{a}$ & 3.0 & 4.0 & 2 & 43.44 \\
\hline 3C 390.3 & 14.3 & $<0.1^{a}$ & 2.3 & 3.9 & $1.7 \pm 0.1$ & 44.67 \\
\hline ESO 140-43 & 14.0 & $1.8^{x}$ & 1.7 & 2.6 & $1.7 \pm 0.2$ & 43.40 \\
\hline IGR J18559+1535 & 12.0 & $0.7^{d}$ & 0.9 & 1.3 & $2.0 \pm 0.2$ & 44.59 \\
\hline ESO $141-55$ & 19.0 & $0.004^{t}$ & 1.9 & 2.8 & $1.9 \pm 0.4$ & 44.30 \\
\hline 1RXS J192450.8-29143 & 4.6 & $0.088^{b}$ & 0.5 & 0.7 & 2 & 45.70 \\
\hline $1 \mathrm{H} 1934-063$ & 9.2 & $0.1^{b}$ & 1.3 & 0.7 & $3.1_{-0.3}^{+0.4}$ & 42.71 \\
\hline IGR J19405-3016 & 11.8 & $<0.1^{\circ}$ & 1.3 & 1.9 & $1.8 \pm 0.2$ & 44.30 \\
\hline NGC 6814 & 16.2 & $<0.05^{a}$ & 3.0 & 4.6 & $1.8 \pm 0.1$ & 42.65 \\
\hline IGR J19473+4452 & 7.1 & $11^{a}$ & 1.1 & 2.0 & $1.3 \pm 0.3$ & 44.33 \\
\hline $3 \mathrm{C} 403$ & 4.5 & $45^{b}$ & 0.9 & 1.2 & 2 & 44.23 \\
\hline QSO B1957+405 & 42.6 & $20^{a}$ & 3.7 & 5.0 & $1.97 \pm 0.05$ & 44.81 \\
\hline ESO 399-20 & 4.3 & $0.048^{b}$ & 0.6 & 0.9 & 2 & 43.33 \\
\hline IGR J20187+4041 & 12.2 & $6.1^{b}$ & 0.9 & 1.3 & $1.9_{-0.2}^{+0.1}$ & 42.99 \\
\hline IGR J20286+2544 & 10.4 & $42^{f}$ & 1.8 & 3.3 & $1.6 \pm 0.2$ & 43.28 \\
\hline Mrk 509 & 11.2 & $<0.01^{a}$ & 3.8 & 4.1 & $2.3 \pm 0.3$ & 44.34 \\
\hline S5 $2116+81$ & 4.4 & $<0.1^{a}$ & 1.3 & 1.8 & 2 & 44.76 \\
\hline IGR J21178+5139 & 7.7 & $2^{f}$ & 0.8 & 1.5 & $1.5 \pm 0.2$ & \\
\hline IGR J21247+5058 & 47.3 & $0.6^{k}$ & 4.7 & 7.3 & $\mathrm{C}$ & 44.04 \\
\hline IGR J21277+5656 & 13.6 & $0.1^{b}$ & 1.6 & 1.8 & $2.2 \pm 0.2$ & 43.20 \\
\hline RX J2135.9+4728 & 9.2 & $0.4^{i}$ & 0.9 & 1.6 & $1.7 \pm 0.2$ & 43.55 \\
\hline PKS 2149-306 & 4.6 & 0.03 & 0.9 & 1.2 & 2 & 47.98 \\
\hline NGC 7172 & 19.4 & $9.0^{a}$ & 3.6 & 4.7 & $2.0 \pm 0.1$ & 43.13 \\
\hline BL Lac & 5.5 & $0.3^{b}$ & 0.7 & 1.2 & $1.8_{-0.3}^{+0.4}$ & 44.34 \\
\hline
\end{tabular}


Table 2. continued.

\begin{tabular}{lrccccc}
\hline \hline Name & $\begin{array}{r}\text { ISGRI } \\
18-60 \mathrm{keV}[\sigma]\end{array}$ & $\begin{array}{c}N_{\mathrm{H}} \\
{\left[10^{22} \mathrm{~cm}^{-2}\right]}\end{array}$ & $\begin{array}{c}f_{20-40 \mathrm{keV}} \\
{\left[10^{-11} \mathrm{erg} \mathrm{cm}^{-2} \mathrm{~s}^{-1}\right]}\end{array}$ & $\begin{array}{c}f_{40-100 \mathrm{keV}} \\
{\left[10^{-11} \mathrm{erg} \mathrm{cm}^{-2} \mathrm{~s}^{-1}\right]}\end{array}$ & $\begin{array}{c}\Gamma_{\text {ISGRI }}^{+} \\
\begin{array}{c}\log L_{20-100 \mathrm{keV}} \\
{\left[\mathrm{erg} \mathrm{s}^{-1}\right]}\end{array}\end{array}$ \\
\hline IGR J22292+6647 & 5.7 & $0.2^{*}$ & 0.5 & 0.6 & $2.3 \pm 0.5$ & 44.57 \\
NGC 7314 & 12.9 & $0.122^{b}$ & 2.1 & 2.5 & $2.2 \pm 0.3$ & 42.37 \\
Mrk 915 & 4.5 & $<0.1^{*}$ & 0.6 & 0.8 & 2 & 43.28 \\
IGR J22517+2217 & 6.6 & $3^{v}$ & 1.6 & 3.4 & $1.4 \pm 0.4$ & 48.43 \\
3C 454.3 & 30.8 & $0.5^{b}$ & 7.1 & 13.2 & $1.58 \pm 0.06$ & 47.76 \\
1H 2251-179 & 22.9 & $<0.19^{a}$ & 3.3 & 3.8 & $2.2 \pm 0.1$ & 44.85 \\
NGC 7469 & 6.1 & $0.061^{b}$ & 1.8 & 2.3 & $2.1 \pm 0.4$ & 44.39 \\
MCG-02-58-022 & 15.0 & $<0.08^{a}$ & 2.0 & 1.4 & $2.8 \pm 0.2$ & 44.26 \\
IGR J23206+6431 & 7.7 & $0.6^{*}$ & 0.4 & 0.7 & 1.5 \\
IGR J23308+7120 & 3.7 & $6^{o}$ & 0.3 & 0.4 & 2 & 44.15 \\
IGR J23524+5842 & 6.7 & $6^{d}$ & 0.4 & 0.5 & $2.0 \pm 0.5$ & 43.39 \\
\hline
\end{tabular}

+ " $\mathrm{C}$ " indicates that a more complex model is required to fit the data (see Table 3); ${ }^{*}$ this work; ${ }^{a}$ Beckmann et al. (2006), and references therein; ${ }^{b}$ Bodaghee et al. (2007), and ref. therein; ${ }^{c}$ Landi et al. (2007b); ${ }^{d}$ Rodriguez et al. (2008); ${ }^{e}$ Malizia et al. (2008); ${ }^{f}$ Malizia et al. (2007); ${ }^{g}$ Beckmann et al. (2007b); ${ }^{h}$ Revnivtsev et al. (2007); ${ }^{i}$ Winter et al. (2008); ${ }^{k}$ Ricci et al. (2009a); ${ }^{l}$ Beckmann et al. (2007a); ${ }^{m}$ Bassani et al. (1999); ${ }^{n}$ Landi et al. (2008); ${ }^{o}$ Landi et al. (2007c); ${ }^{p}$ Molina et al. (2008); ${ }^{q}$ Sazonov et al. (2008); ${ }^{s}$ Landi et al. (2007a); ${ }^{t}$ Gondoin et al. (2003); ${ }^{u}$ Landi et al. (2007d); ${ }^{v}$ Bassani et al. (2007); ${ }^{w}$ Tomsick et al. (2008); ${ }^{x}$ Ricci et al. (2009b).

Table 3. Spectral fit of a cut-off power-law model for AGN with more complex IBIS/ISGRI spectra.

\begin{tabular}{lcccc}
\hline \hline Name & $\begin{array}{c}N_{\mathrm{H}} \\
{\left[10^{22} \mathrm{~cm}^{-2}\right]}\end{array}$ & $\Gamma_{\text {ISGRI }}$ & $\begin{array}{c}E_{\mathrm{C}} \\
{[\mathrm{keV}]}\end{array}$ & $\chi_{v}^{2}$ (d.o.f.) \\
\hline Mrk 348 & 30 & $0.9_{-0.6}^{+0.4}$ & $55_{-25}^{+56}$ & $0.91(8)$ \\
NGC 4151 & 6.9 & $1.60_{-0.07}^{+0.06}$ & $118_{-13}^{+21}$ & $1.10(9)$ \\
NGC 4388 & 27 & $1.3^{0.1}$ & $95_{-17}^{+26}$ & $0.34(7)$ \\
NGC 4507 & 29 & $1.15_{-0.13}^{+0.28}$ & $72_{-22}^{+53}$ & $0.54(8)$ \\
NGC 4593 & 0.02 & $1.1_{-0.2}^{+0.4}$ & $48_{-9}^{+114}$ & $0.87(7)$ \\
NGC 4945 & 400 & $1.43_{-0.11}^{+0.16}$ & $127_{-26}^{+56}$ & $0.75(8)$ \\
4U 1344-60 & 5 & $1.11_{-0.09}^{+0.29}$ & $51_{-12}^{+27}$ & $0.76(7)$ \\
IC 4329A & 0.42 & $1.37_{ \pm}^{+0.17}$ & $80_{-19}^{+31}$ & $1.50(9)$ \\
Circinus Galaxy & 360 & $1.29_{-0.13}^{+0.18}$ & $33_{-3}^{+6}$ & $1.03(7)$ \\
GRS 1734-292 & 3.7 & $1.50 \pm 0.10$ & $54_{-7}^{+8}$ & $0.88(8)$ \\
IGR J17488-3253 & 0.2 & $0.74_{-0.12}^{+0.33}$ & $40_{-3}^{+8}$ & $0.98(7)$ \\
IGR J21247+5058 & 0.6 & $1.19_{-0.19}^{+0.13}$ & $74_{-28}^{+38}$ & $0.75(8)$ \\
\hline
\end{tabular}

i.e. removing $\frac{1}{4}$ pixel from each corner (standard output from OSA). Therefore the computed values include the contributions by any other source inside the photometric aperture. We flagged in Table 5 those sources that might be affected by a nearby star (at less than $1^{\prime}$ ), with a potential contamination below $0.2 \mathrm{mag}$ in any case. Other 8 AGN containing a brighter contaminating source within the extraction aperture were not included in this compilation. In addition, for some extended AGN, this $3 \times$ 3 aperture does not cover the full galaxy size, but just their central region.

To only include high-quality data, some selection criteria were applied to individual photometric points. Shots were checked against saturation, rejecting those with long exposures for the brightest sources, if necessary. For faint sources, a minimum signal-to-noise ratio of 3 was required for the longest integration shots. The shortest shots were only used if the signalto-noise ratio was greater than 10 . Because these sources can show extended structure in the OMC images, anomalous PSF, as well as problems in the centroid determination, were allowed. Finally, to avoid contamination by cosmic rays, we excluded those points whose fluxes deviate more than 5 times the standard deviation from the median value of their surrounding points, applying three iterations of this filter.

We list in Table 5 the median $V$ magnitude of each AGN, the average of error estimates ( $1 \sigma$ level) of each photometric point given by OSA $7,\left\langle\sigma_{V}\right\rangle$, the luminosity in the Johnson $V$ filter (centred on $5500 \AA$, effective width $890 \AA$ ), the $\alpha_{\text {OX }}$ value, the number of photometric points used in the analysis and a flag indicating the potential contamination of the photometric value by a nearby star. The value of $\alpha_{\mathrm{OX}}$ is measured as the slope of a power law between the two energy ranges

$\alpha_{\mathrm{OX}}=-\frac{\log \left(f_{\mathrm{O}} / f_{\mathrm{X}}\right)}{\log \left(v_{\mathrm{O}} / v_{\mathrm{X}}\right)}$.

Here, $f_{\mathrm{O}}$ and $f_{\mathrm{X}}$ are the monochromatic fluxes at the frequencies $v_{O}($ at $5500 \AA)$ and $v_{\mathrm{X}}($ at $20 \mathrm{keV})$.

No $K$ correction has been applied to the $V$ luminosities, since the redshifts are relatively low and the optical slope of these objects is not well known. Moreover, depending on the redshift, the $V$ band might be contaminated to different degrees by the OIII and $\mathrm{H} \beta$ emission lines. We did not correct for this effect, either.

\subsubsection{Optical variability}

Among the 57 AGN for which OMC data are available, 3 show strong variability in the photometric $V$-band data, with an amplitude larger than 0.5 mag: QSO B0716+714, NGC 4151, and 3C 279.

The BL Lac QSO B0716+714 appears as a point source in a low background field and was monitored by OMC during 2 periods, at IJD $^{3}$ around 1415 (November 2003) and 1555 (April 2004). This source brightened by a factor $\sim 4$ during this period, as shown in Fig. 2, ranging from $V=14.60 \mathrm{mag}$ to 13.05 mag. Moreover, this blazar also shows a day-scale variability pattern within the two monitoring periods, with an amplitude around $0.3 \mathrm{mag}$.

NGC 4151 is an extended source, classified as Seyfert 1.5, much larger than the OMC aperture. We detected a clear weakening of its central, dominating region by around 0.5 mag (60\% in flux) from 11.15 mag to 11.65 mag between May 2003

\footnotetext{
3 The INTEGRAL Julian Date is defined as IJD = MJD-51 544.0. The origin of IJD is 2000 January 1 expressed in Terrestrial Time.
} 
Table 4. Spectral fit results for combined JEM-X and IBIS/ISGRI data.

\begin{tabular}{|c|c|c|c|c|c|c|c|c|}
\hline Name & $\begin{array}{r}\text { JEM-X } \\
{[\sigma]} \\
\end{array}$ & $\begin{array}{c}N_{\mathrm{H}}^{b} \\
{\left[10^{22} \mathrm{~cm}^{-2}\right]}\end{array}$ & $f_{3-20 \mathrm{keV}}^{c}$ & $f_{20-100 \mathrm{keV}}^{c}$ & $\Gamma$ & $\begin{array}{c}E_{\mathrm{C}} \\
{[\mathrm{keV}]}\end{array}$ & $\begin{array}{c}\log L_{3-100 \mathrm{keV}} \\
{\left[\mathrm{erg} \mathrm{s}^{-1}\right]}\end{array}$ & $\chi_{v}^{2}$ (d.o.f.) \\
\hline 1ES $0033+59.5$ & 10.1 & 0.36 & 6.0 & 1.4 & $2.75_{-0.16}^{+0.18}$ & - & 45.16 & $0.51(8)$ \\
\hline NGC $1275^{a}$ & 38.6 & 3.75 & 22.8 & 3.0 & -0.16 & - & 43.58 & $>5^{a}$ \\
\hline $3 \mathrm{C} 120$ & 5.5 & 0.2 & 5.8 & 7.5 & $1.76 \pm 0.06$ & - & 44.52 & $0.67(15)$ \\
\hline Mrk 421 & 155.7 & 0.08 & 61.6 & 32.5 & $2.12_{-0.04}^{+0.05}$ & $133_{-21}^{+32}$ & 45.29 & $1.43(14)$ \\
\hline NGC $4151^{a}$ & 91.5 & 6.9 & 33.2 & 40.4 & $1.75_{-0.06}^{+0.04}$ & $170_{-32}^{+32}$ & 43.25 & $1.36(14)$ \\
\hline NGC $4388^{a}$ & 14.3 & 27 & 10.3 & 26.0 & $1.26_{-0.16}^{+0.15}$ & $78_{-20}^{-35^{2}}$ & 43.68 & $0.41(15)$ \\
\hline NGC $4593^{a}$ & 7.0 & 0.02 & 4.4 & 7.7 & $1.46_{-0.15}^{+0.09}$ & $193_{-93}^{-12}$ & 43.34 & $1.01(15)$ \\
\hline $\operatorname{Cen~}^{a}$ & 57.2 & 12.5 & 28.1 & 43.4 & $1.85 \pm 0.01$ & $-^{-90}$ & 42.94 & 1.49 (17) \\
\hline MCG-06-30-015 & 11.2 & 0.03 & 7.0 & 5.0 & $2.10 \pm 0.05$ & - & 42.93 & 0.79 (10) \\
\hline $4 \mathrm{U} 1344-60^{a}$ & 8.3 & 5 & 5.6 & 21.0 & $1.47_{-0.16}^{+0.11}$ & $86_{-22}^{+31}$ & 43.70 & $0.76(15)$ \\
\hline IC $4329 \mathrm{~A}^{a}$ & 11.3 & 0.42 & 14.5 & 21.1 & $1.41 \pm 0.08$ & $86_{-13}^{+12}$ & 44.31 & $1.29(15)$ \\
\hline Circinus Galaxy $^{a}$ & 5.3 & 360 & 3.9 & 21.0 & $1.2 \pm 0.2$ & $30 \pm 3$ & 42.03 & $1.52(11)$ \\
\hline NGC 5506 & 16.8 & 3.4 & 12.1 & 8.0 & $2.20 \pm 0.08$ & - & 43.27 & $0.66(12)$ \\
\hline H $1426+428$ & 7.5 & $<0.02$ & 6.1 & 1.2 & $2.83_{-0.21}^{+0.25}$ & - & 44.90 & $0.67(9)$ \\
\hline Mrk 501 & 7.9 & 0.013 & 10.2 & 4.8 & $2.3 \pm 0.1$ & - & 44.60 & $0.73(15)$ \\
\hline GRS $1734-292^{a}$ & 10.5 & 3.7 & 6.7 & 8.0 & $1.25_{-0.16}^{+0.10}$ & $39_{-6}^{+5}$ & 44.02 & $1.28(12)$ \\
\hline IGR J17488-3253 ${ }^{a}$ & 21.8 & 0.72 & 1.7 & 2.9 & $1.63 \pm 0.03$ & -6 & 43.11 & $1.10(119)$ \\
\hline QSO B1957+405 ${ }^{a}$ & 10.5 & 20 & 16.2 & 8.3 & $1.9_{-0.2}^{+0.1}$ & - & 45.26 & $0.76(9)$ \\
\hline Mrk 509 & 7.2 & $<0.01$ & 7.5 & 9.3 & $1.8 \pm 0.1$ & - & 44.66 & $0.69(8)$ \\
\hline IGR J21178+5139 & 17.1 & 2 & 1.5 & 2.2 & $1.7 \pm 0.1$ & - & - & $1.61(13)$ \\
\hline IGR J21247+5058 & 54.1 & 0.6 & 9.5 & 11.1 & $1.4 \pm 0.1$ & $61_{-11}^{+22}$ & 44.27 & $1.41(11)$ \\
\hline $3 \mathrm{C} 454.3$ & 6.4 & 0.5 & 10.7 & 20.3 & $1.54 \pm 0.05$ & - & 47.93 & $0.58(16)$ \\
\hline $1 \mathrm{H} 2251-179$ & 10.7 & $<0.19$ & 5.7 & 7.2 & $1.8 \pm 0.1$ & - & 45.10 & $1.20(13)$ \\
\hline
\end{tabular}

${ }^{a}$ see Appendix A; ${ }^{b} N_{\mathrm{H}}$ values have been fixed during the fit; ${ }^{c}$ fluxes are given in $\left[10^{-11} \mathrm{erg} \mathrm{cm}^{-2} \mathrm{~s}^{-1}\right]$

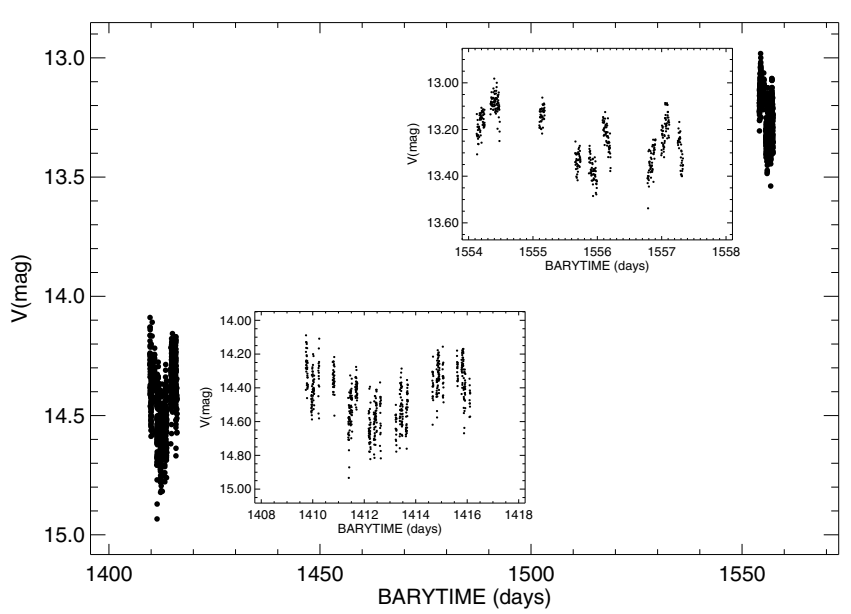

Fig. 2. OMC lightcurve of QSO B0716+714. The insets show a zoom on the lightcurves during the 2 periods when this object was monitored by OMC. The optical luminosity increased by a factor of 4 . The photometric accuracy of individual photometric points is $\sigma \sim 0.09$ mag. Barycentric time is given in INTEGRAL Julian Date (IJD).

and January 2007. Since the optical photometry is contaminated to some extent by bulge stellar light, the variation in the optical emission from the nucleus itself might have been significantly larger.

The AGN 3C 279 was barely detectable around June 2003, with a $2 \sigma$ OMC detection at $V=17.0$. The catalogued value for it in the low state is $V=17.8 \mathrm{mag}$ (O'Dell et al. 1978). After May 2005, this blazar was clearly detected with a brightness in the range $V=14.8-15.8 \mathrm{mag}$, indicating that it brightened by up to a factor close to 10 (Fig. 3). This is a very active source, with optical photometry reported in the range $B=18.3$ to $B=11.3$ (O'Dell et al. 1978).

Other AGN monitored by OMC also show some hints of variability, such as 3C 273 and 3C 390.3, but at smaller amplitudes (just a few percent over the period considered).

\section{Properties of INTEGRAL detected AGN}

The catalogue of INTEGRAL-detected extragalactic objects presented here comprises 187 sources in total. Out of this sample, 162 objects have been identified as Seyfert galaxies (161 with redshift information), 18 blazars (all of them with redshift), and 7 objects have been claimed to be AGN without further specification of the AGN type. Within the Seyfert group, we found 67 Seyfert 1 to 1.2 objects, 29 intermediate Seyfert 1.5, and 66 Seyfert type 1.9 and type 2 .

The redshift distribution of the Seyfert type AGN in this sample is shown in Fig. 4. The average redshift is $z=0.03$. Figure 5 shows the parameter space filled by INTEGRAL-detected AGN in redshift and X-ray luminosity, ranging from low-luminous low-redshift Seyfert as close as $z=0.001$ up to the high-redshift blazar domain.

To investigate the spectra of AGN subtypes, we derived averaged spectral properties and stacked spectra of the Seyfert 1 and 2 types, as well as for the intermediate Seyferts and the blazars, and according to the intrinsic absorption. The nine brightest sources, two blazars and seven Seyfert, with $>50 \sigma$ (Cen A, NGC 4151, GRS 1734-292, NGC 4945, NGC 4388, IC 4329A, Circinus Galaxy, 3C 273, and Mrk 421) have been excluded from the statistical spectral analyses because their high signalto-noise ratio would dominate the averaged spectra, and also all sources with $<5 \sigma$ were ignored. In addition, we excluded the 
Table 5. Median $V$ magnitude, the average of error estimates $\left\langle\sigma_{V}\right\rangle$, luminosity in the Johnson $V$ filter, $\alpha$ OX, the number of photometric points and contamination flag ("Y" indicates potential contamination by a nearby star up to $0.2 \mathrm{mag}$; see text for details).

\begin{tabular}{|c|c|c|c|c|c|c|}
\hline Name & $V[\mathrm{mag}]$ & $\left\langle\sigma_{V}\right\rangle[\mathrm{mag}]$ & $\log L_{V}\left[\operatorname{erg~s}^{-1}\right]$ & $\alpha_{\mathrm{OX}}$ & $N$ & Cont. flag \\
\hline Mrk 348 & 13.76 & 0.05 & 42.71 & 1.01 & 81 & $\mathrm{Y}$ \\
\hline NGC 788 & 12.60 & 0.04 & 43.09 & 1.14 & 1342 & \\
\hline NGC 985 & 13.73 & 0.05 & 43.66 & 1.13 & 644 & \\
\hline NGC 1052 & 11.38 & 0.03 & 42.70 & 1.31 & 1004 & \\
\hline NGC 1068 & 9.99 & 0.02 & 44.80 & 1.38 & 693 & \\
\hline NGC 1275 & 12.51 & 0.04 & 43.27 & 1.21 & 856 & \\
\hline NGC 1365 & 11.51 & 0.05 & 42.72 & 1.27 & 35 & \\
\hline $3 \mathrm{C} 120$ & 14.00 & 0.05 & 43.31 & 1.02 & 216 & \\
\hline UGC 3142 & 15.12 & 0.22 & 43.49 & 0.93 & 259 & \\
\hline ESO 33-2 & 13.99 & 0.09 & 42.78 & 1.09 & 40 & \\
\hline Mrk 3 & 12.88 & 0.04 & 42.95 & 1.08 & 425 & $\mathrm{Y}$ \\
\hline Mrk 6 & 13.68 & 0.06 & 42.94 & 1.07 & 657 & \\
\hline QSO B0716+714 & 14.27 & 0.09 & 45.26 & 1.19 & 1116 & \\
\hline ESO 209-12 & 14.60 & 0.21 & 43.24 & 1.07 & 387 & \\
\hline PG 0804+761 & 14.07 & 0.08 & 44.28 & 1.09 & 299 & \\
\hline 4U 0937-12 & 12.66 & 0.03 & 42.56 & 1.12 & 443 & \\
\hline MCG-05-23-016 & 13.12 & 0.05 & 42.47 & 1.03 & 1514 & \\
\hline NGC 3227 & 12.06 & 0.04 & 42.21 & 1.11 & 12 & \\
\hline NGC 3281 & 12.67 & 0.04 & 42.85 & 1.16 & 316 & \\
\hline Mrk 421 & 13.05 & 0.06 & 43.58 & 0.91 & 3112 & \\
\hline NGC 4051 & 12.26 & 0.04 & 41.67 & 1.20 & 76 & \\
\hline NGC 4138 & 11.87 & 0.04 & 42.06 & 1.26 & 585 & \\
\hline NGC 4151 & 11.52 & 0.04 & 42.28 & 1.03 & 7461 & \\
\hline Mrk 50 & 14.47 & 0.09 & 42.86 & 1.11 & 774 & $\mathrm{Y}$ \\
\hline NGC 4388 & 12.26 & 0.06 & 42.80 & 1.05 & 509 & \\
\hline NGC 4395 & 14.09 & 0.11 & 40.23 & 1.05 & 7 & \\
\hline $3 C 273$ & 12.58 & 0.04 & 45.31 & 1.05 & 2010 & $\mathrm{Y}$ \\
\hline IGR J12391-1612 & 14.15 & 0.06 & 43.34 & 1.05 & 104 & \\
\hline NGC 4593 & 12.24 & 0.04 & 42.83 & 1.14 & 1204 & \\
\hline ESO 323-32 & 13.11 & 0.07 & 43.03 & 1.20 & 158 & $\mathrm{Y}$ \\
\hline $3 \mathrm{C} 279$ & 15.52 & 0.11 & 45.29 & 1.01 & 294 & \\
\hline Mrk 783 & 15.39 & 0.16 & 43.39 & 1.01 & 37 & \\
\hline NGC 4945 & 12.44 & 0.17 & 41.43 & 1.04 & 327 & \\
\hline IGR J13091+1137 & 13.70 & 0.05 & 43.18 & 1.07 & 15 & \\
\hline NGC 5033 & 11.57 & 0.03 & 42.16 & 1.33 & 55 & \\
\hline ESO 383-18 & 14.33 & 0.08 & 42.32 & 1.09 & 673 & $\mathrm{Y}$ \\
\hline MCG-06-30-015 & 13.23 & 0.07 & 42.36 & 1.09 & 1268 & $\mathrm{Y}$ \\
\hline Mrk 268 & 14.30 & 0.06 & 43.37 & 1.08 & 7 & \\
\hline IC 4329A & 13.20 & 0.05 & 43.00 & 0.98 & 501 & \\
\hline NGC 5506 & 12.93 & 0.05 & 42.38 & 1.03 & 701 & \\
\hline NGC 5548 & 13.14 & 0.05 & 43.08 & 1.17 & 256 & \\
\hline H $1426+428$ & 16.11 & 0.25 & 43.72 & 0.98 & 619 & \\
\hline NGC 5995 & 13.47 & 0.07 & 43.29 & 1.05 & 118 & $\mathrm{Y}$ \\
\hline ESO 138-1 & 13.59 & 0.11 & 42.34 & 1.20 & 12 & $\mathrm{Y}$ \\
\hline NGC 6221 & 11.91 & 0.07 & 42.49 & 1.33 & 16 & \\
\hline Mrk 501 & 13.21 & 0.04 & 43.65 & 1.07 & 567 & \\
\hline NGC 6300 & 11.52 & 0.05 & 42.38 & 1.19 & 31 & \\
\hline 3С 390.3 & 14.61 & 0.13 & 43.54 & 1.00 & 482 & $\mathrm{Y}$ \\
\hline 1H 1934-063 & 13.35 & 0.05 & 42.59 & 1.10 & 12 & \\
\hline NGC 6814 & 12.42 & 0.04 & 42.31 & 1.14 & 134 & \\
\hline ESO 399-20 & 13.96 & 0.08 & 43.07 & 1.18 & 84 & \\
\hline Mrk 509 & 13.38 & 0.05 & 43.60 & 1.03 & 400 & \\
\hline NGC 7172 & 12.66 & 0.04 & 42.66 & 1.10 & 508 & \\
\hline Mrk 915 & 14.10 & 0.09 & 42.75 & 1.09 & 40 & \\
\hline $1 \mathrm{H} 2251-179$ & 14.20 & 0.09 & 43.83 & 0.98 & 1570 & $\mathrm{Y}$ \\
\hline NGC 7469 & 12.70 & 0.04 & 44.21 & 1.15 & 22 & \\
\hline MCG-02-58-022 & 14.13 & 0.06 & 43.59 & 1.01 & 93 & \\
\hline
\end{tabular}

5 Compton thick objects, i.e. NGC 1068, NGC 3281, NGC 4945, Circinus Galaxy, ESO 138-1.

The average Seyfert 1 (including type 1.2) spectral property was constructed using the mean weighted by the errors on the photon indices of 55 ISGRI power-law fit results, the
Seyfert 2 composite spectrum includes 44 sources, and $20 \mathrm{ob}-$ jects form the intermediate Seyfert 1.5 group where spectral fitting allowed constraining the spectral shape (Table 2). In addition, 11 blazars allowed spectral extraction. When computing the weighted average of the various subclasses, the 11 blazars 


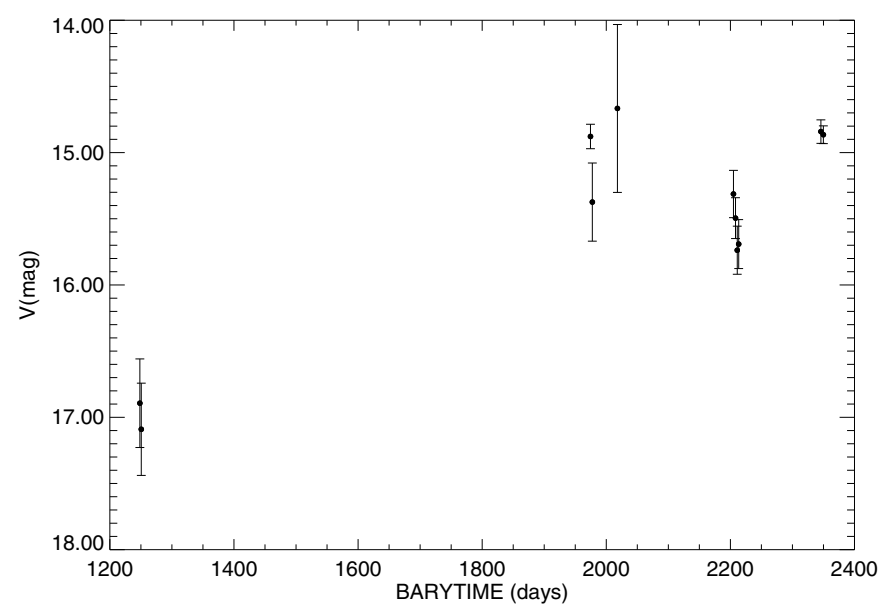

Fig. 3. OMC lightcurve of 3C 279. Barycentric time is given in IJD. Photometric data have been re-binned to obtain one value per revolution (3 days).

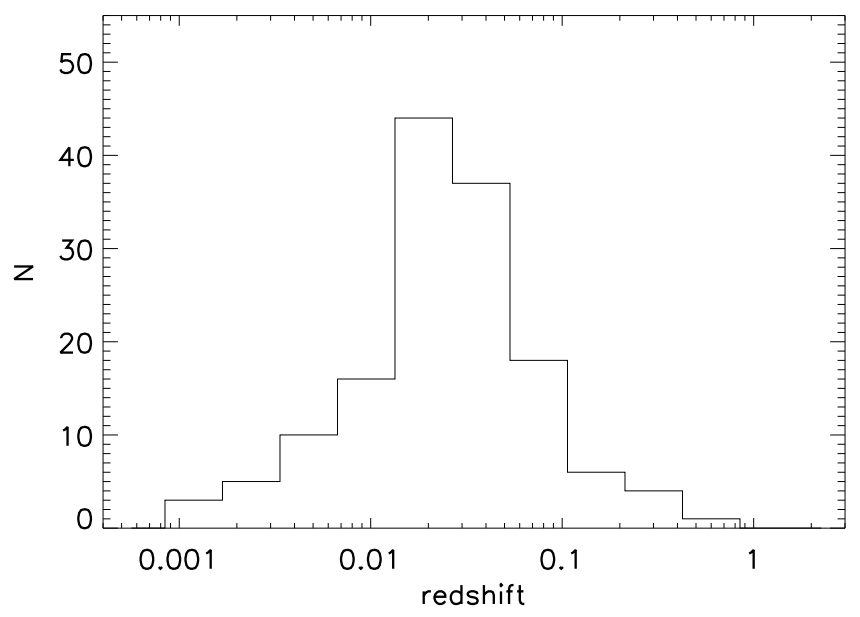

Fig. 4. Redshift distribution of the Seyfert content of 144 INTEGRAL detected AGN with detection significance $\geq 4 \sigma$. The average redshift is $z=0.03$.

had a hard X-ray spectrum with $\Gamma=1.55 \pm 0.04$ when compared to the 119 Seyfert galaxies with $\Gamma=1.93 \pm 0.01$. The Seyfert $1(\Gamma=1.92 \pm 0.02)$ and Seyfert $1.5(\Gamma=2.02 \pm$ 0.03 ) only show slightly steeper hard X-ray spectra than the Seyfert 2 objects $(\Gamma=1.88 \pm 0.02)$. Table 6 gives the properties of the different Seyfert types. All quantities, except for the photon indices, have been averaged in logarithmic space. For 12 objects, a cut-off power law model gave a better representation of the ISGRI spectra (Table 3 ). The average photon index is in these cases $\langle\Gamma\rangle=1.3 \pm 0.4$ with a cut-off energy of $\left\langle E_{\mathrm{C}}\right\rangle=86 \pm 25 \mathrm{keV}$.

We get a similar result when stacking the IBIS/ISGRI spectra together. Again, only sources above $5 \sigma$ are considered here. The spectra were renormalised on the $18-30 \mathrm{keV}$ energy bin before stacking them, re-adjusting the errors on the flux so that the significance is taken into account. The significances for the stacked spectra are consistent with what would be expected based on the single spectra significances, i.e. the $18-60 \mathrm{keV}$ significances are $98 \sigma$ for the Seyfert 1, 61 $\sigma$ (Seyfert 1.5), 94 $\sigma$ (Seyfert 2), and $148 \sigma$ for all Seyfert spectra stacked together. The results of spectral model fitting to these spectra are summarized in Table 7. A simple power law model gives a photon index of $\Gamma=1.97 \pm$ 0.02 for all Seyfert objects, $\Gamma=1.96_{-0.02}^{+0.03}$ for the Seyfert 1,

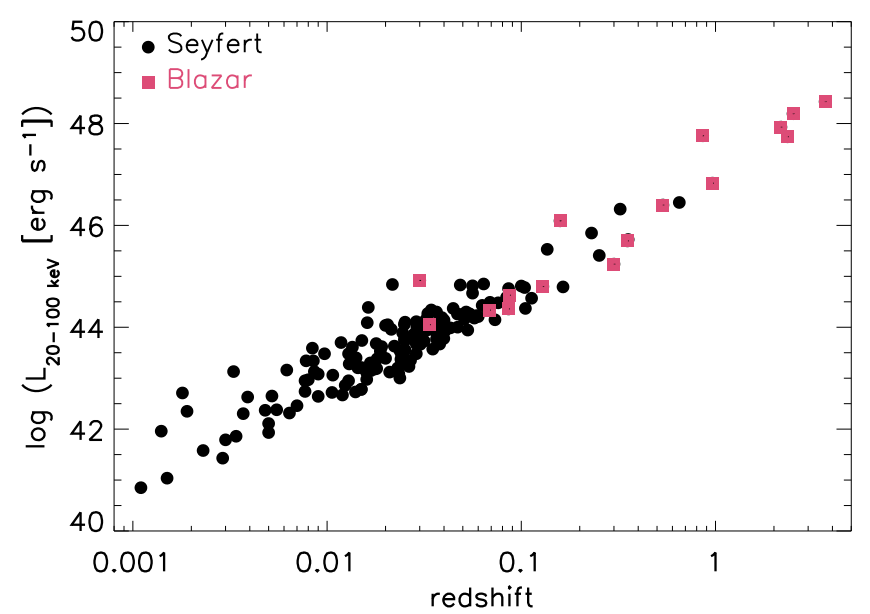

Fig. 5. X-ray luminosity versus redshift of 161 INTEGRAL detected AGN with significance $\geq 4 \sigma$.

Table 6. Average properties of the INTEGRAL AGN. In parentheses, the number of objects used for the given average value is indicated.

\begin{tabular}{|c|c|c|c|}
\hline & Seyfert 1 & Seyfert 1.5 & Seyfert 2 \\
\hline$\langle z\rangle$ & $0.03(63)$ & $0.014(24)$ & $0.02(57)$ \\
\hline$\langle\Gamma\rangle^{a}$ & $\begin{array}{c}1.92 \pm 0.02 \\
(55)\end{array}$ & $\begin{array}{c}2.02 \pm 0.03 \\
(20)\end{array}$ & $\begin{array}{c}1.88 \pm 0.02 \\
(44)\end{array}$ \\
\hline$\left\langle\log N_{\mathrm{H}}\right\rangle^{b}$ & $21.2(61)$ & $21.7(23)$ & $22.9(51)$ \\
\hline$\left\langle\log L_{20-100 \mathrm{keV}}\right\rangle^{c}$ & $44.0(63)$ & $43.3(24)$ & $43.4(57)$ \\
\hline$\left\langle\log M_{\mathrm{BH}}\right\rangle$ & $7.8(30)$ & $7.2(14)$ & $7.7(27)$ \\
\hline \multirow[t]{2}{*}{$\langle\lambda\rangle^{d}$} & $0.064(30)$ & 0.015 (14) & $0.02(27)$ \\
\hline & Unabs & Absorbed & all Sey \\
\hline$\langle z\rangle$ & $0.03(74)$ & $0.014(60)$ & $0.02(144)$ \\
\hline$\langle\Gamma\rangle^{a}$ & $\begin{array}{c}1.94 \pm 0.02 \\
(66)\end{array}$ & $\begin{array}{c}1.91 \pm 0.02 \\
(44)\end{array}$ & $\begin{array}{c}1.93 \pm 0.01 \\
(119)\end{array}$ \\
\hline$\left\langle\log N_{\mathrm{H}}\right\rangle^{b}$ & $21.0(75)$ & $23.1(60)$ & $21.9(135)$ \\
\hline$\left\langle\log L_{20-100 \mathrm{keV}}\right\rangle^{c}$ & $43.8(74)$ & $43.4(60)$ & 43.6 (144) \\
\hline$\left\langle\log M_{\mathrm{BH}}\right\rangle$ & $7.6(37)$ & $7.7(34)$ & $7.6(71)$ \\
\hline$\langle\lambda\rangle^{d}$ & $0.06(37)$ & $0.015(34)$ & $0.03(71)$ \\
\hline
\end{tabular}

${ }^{a} \Gamma$ is the average photon index derived from the weighted mean on the power law model fits to the single IBIS/ISGRI spectra; ${ }^{b}$ absorption is given in $\left[\mathrm{cm}^{-2}\right] ;{ }^{c}$ luminosities in $\left[\mathrm{erg} \mathrm{s}^{-1}\right] ;{ }^{d} \lambda=L_{\mathrm{Bol}} / L_{\mathrm{Edd}}$ is the Eddington ratio.

$\Gamma=2.02 \pm 0.04$ for Seyfert 1.5 , and $\Gamma=1.89_{-0.02}^{+0.04}$ for the Seyfert 2 class. In all cases, a cut-off power law improves the fit result significantly according to an F-test (Table 7). The resulting model is $\Gamma=1.4 \pm 0.1$ with cut-off at $E_{\mathrm{C}}=86_{-17}^{+16} \mathrm{keV}$ for all Seyfert galaxies, fully consistent with the average from the 12 cut-off power law model fits. For Seyfert 1 we derive $\Gamma=1.4 \pm 0.1$ and $E_{\mathrm{C}}=86_{-14}^{+21} \mathrm{keV}$, and $\Gamma=1.4 \pm 0.2$ with $E_{\mathrm{C}}=63_{-12}^{+20} \mathrm{keV}$ for intermediate Seyfert 1.5 , and $\Gamma=1.65 \pm$ 0.05 with $E_{\mathrm{C}}=184_{-52}^{+16} \mathrm{keV}$ Seyfert 2 galaxies. It should be considered that the latter fit still has a poor quality with $\chi_{v}^{2}=2.6$.

A model that adds a reflection component from cold material to the underlying continuum (the so-called PEXRAV model; Magdziarz \& Zdziarski 1995) gives again a better fit in most cases. The underlying continuum shows a similar gradient in the different source classes when not allowing for a high-energy cutoff in the PEXRAV model, so this fit has the same degree of freedom as the one using the cut-off power law. The difference shows up, however, in the inclination angle $i$ and strength $R$ of the reflection component. Here $R$ is defined as the relative amount of reflection compared to the directly viewed primary spectrum. 
Table 7. Results from spectral fitting of the stacked IBIS/ISGRI spectra of INTEGRAL AGN

\begin{tabular}{ccccc}
\hline \hline Sample & $\Gamma$ & $E_{\mathrm{C}}[\mathrm{keV}]$ & $R$ & $\chi_{v}^{2}$ \\
\hline Sey $1(\geq 5 \sigma)$ & $1.96_{-0.02}^{+0.03}$ & - & - & 5.66 \\
& $1.44 \pm 0.10$ & $86_{-14}^{+21}$ & - & 1.10 \\
$\left(i=30^{\circ}\right)$ & $1.96 \pm 0.02$ & - & $1.2_{-0.3}^{+0.6}$ & 1.15 \\
\hline Sey $1.5(\geq 5 \sigma)$ & $2.02 \pm 0.04$ & - & - & 3.54 \\
& $1.36 \pm 0.15$ & $63_{-12}^{+20}$ & - & 0.57 \\
$\left(i=45^{\circ}\right)$ & $2.04 \pm 0.04$ & - & $3.1_{-1.3}^{+4.7}$ & 0.29 \\
\hline Sey 2 $(\geq 5 \sigma)$ & $1.89_{-0.02}^{+0.04}$ & - & - & 3.13 \\
& $1.65_{0.05}$ & $184_{-52}^{+16}$ & - & 2.58 \\
$\left(i=60^{\circ}\right)$ & $1.91_{-0.03}^{+0.02}$ & - & $1.1_{-0.4}^{+0.7}$ & 1.67 \\
\hline all Sey $(\geq 5 \sigma)$ & $1.97 \pm 0.02$ & - & - & 6.18 \\
& $1.44_{-0.13}^{+0.08}$ & $86_{-17}^{+16}$ & - & 1.96 \\
$\left(i=45^{\circ}\right)$ & $1.95 \pm 0.02$ & - & $1.3_{-0.4}^{+0.7}$ & 1.53 \\
\hline all Sey $(\geq 10 \sigma)$ & $1.98 \pm 0.02$ & - & - & 6.04 \\
& $1.41_{-0.11}^{+0.10}$ & $80_{-13}^{+17}$ & - & 1.23 \\
$\left(i=45^{\circ}\right)$ & $1.95 \pm 0.02$ & - & $1.3_{-0.4}^{+0.7}$ & 1.23 \\
\hline Unabs. $(\geq 5 \sigma)$ & $1.97_{-0.01}^{+0.03}$ & - & - & 5.64 \\
& $1.53_{-0.08}^{+0.09}$ & $100_{-15}^{+25}$ & - & 1.82 \\
$\left(i=45^{\circ}\right)$ & $1.98 \pm 0.02$ & - & $1.3_{-0.4}^{+0.6}$ & 0.95 \\
\hline Abs. $(\geq 5 \sigma)$ & $1.91_{-0.03}^{+0.04}$ & - & - & 1.2 \\
& $1.43_{-0.08}^{+0.03}$ & $94_{-13}^{+32}$ & - & 1.59 \\
$\left(i=45^{\circ}\right)$ & $1.91_{-0.03}^{+0.02}$ & - & $1.5_{-1.4}^{+1.5}$ & 1.03 \\
\hline & & & &
\end{tabular}

The value of $R$ depends on the inclination angle $i$ between the normal of the accretion disc and the line of sight. The smaller the inclination angle, the larger the resulting reflection component. As the data are not sufficient to fit $R$ and $i$ simultaneously, the inclination angle was set to $i=30^{\circ}$ for Seyfert $1, i=45^{\circ}$ for Seyfert 1.5 , and $i=60^{\circ}$ for Seyfert 2. It is worth noting that the quality of the fit did not depend on the choice of $i$. The results are included in Table 7. Using this model, Seyfert 1 and Seyfert 2 show only slightly different underlying continua and a reflection component of the same strength $R \simeq 1$ within the statistical errors. Seyfert 1.5 objects appear to have slightly steeper spectra $(\Gamma=2.0)$ and stronger reflection $\left(R=3_{-1}^{+5}\right)$. Applying a higher significance level (e.g. $\geq 10 \sigma$ ) for the source selection does not change the results significantly, as shown for all Seyfert galaxies in Table 7. This confirms that the stacked spectra are dominated by the most significant sources. The result of the fit to the whole Seyfert sample with the PEXRAV model is shown in Fig. 6.

The classification according to the Seyfert type of the objects is based on optical observations. An approach to classifying sources due to their properties in the X-rays can be done by separating the sources with high intrinsic absorption $\left(N_{\mathrm{H}}>10^{22} \mathrm{~cm}^{-2}\right)$ from those objects that do not show significant absorption in the soft X-rays. Not all objects that show high intrinsic absorption in the X-rays are classified as Seyfert 2 galaxies in the optical, and the same applies to the other AGN sub-types. Nevertheless a similar trend in the spectral slopes can be seen: the 44 absorbed AGN show a hard X-ray spectrum $(\langle\Gamma\rangle=1.91 \pm 0.02)$ consistent with that of the 66 unabsorbed sources $(\langle\Gamma\rangle=1.94 \pm 0.02)$. Using the stacked spectra, the absorbed sources show a slightly flatter continuum with $\Gamma=$ $1.91_{-0.03}^{+0.04}$ than unabsorbed Seyfert galaxies, with $\Gamma=1.97_{-0.01}^{+0.03}$. Also here, a cut-off power law has been tested, but comparing it to the simple power law model improves the fit only for the

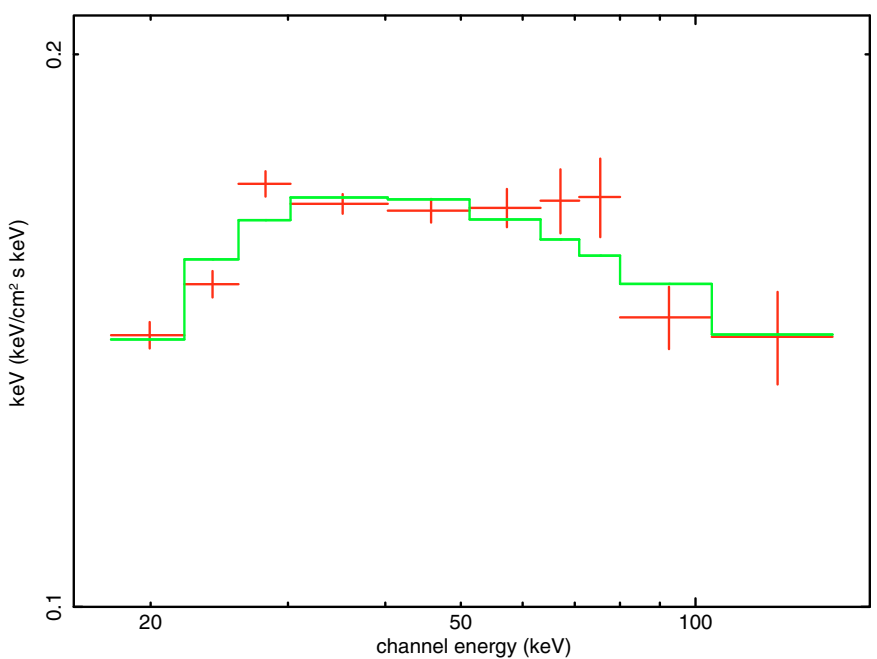

Fig. 6. Stacked IBIS/ISGRI spectrum for Seyfert objects (excluding Compton thick sources) in $E F_{E}$ versus energy. The spectrum has been fit by a PEXRAV model with $\Gamma=1.95$ and reflection $R=1.3$.

unabsorbed sources. For these sources we derive $\Gamma=1.5 \pm 0.1$ and $E_{\mathrm{C}}=100_{-15}^{+25} \mathrm{keV}$, and for the absorbed ones $\Gamma=1.4 \pm 0.1$ with $E_{\mathrm{C}}=94_{-13}^{+32} \mathrm{keV}$. Thus, for the different absorption classes we get consistent cut-off values and spectral slopes, although the stacked unabsorbed spectrum is represented better by the simple power-law model with no cut-off. Applying the PEXRAV model shows that the spectra can be represented by reflection models. The fit improves significantly when adding a reflection component with $R \simeq 1.5$, while the underlying continuum slope is the same as for the simple power law.

A difference between type 1 and type 2 objects is seen in the average luminosity of these subclasses. For 60 absorbed Seyfert galaxies, the average luminosity is $\left\langle L_{20-100 \mathrm{keV}}\right\rangle=2.5 \times$ $10^{43} \mathrm{erg} \mathrm{s}^{-1}$, more than a factor of 2 lower than for the 74 unabsorbed Seyfert with redshift information $\left(\left\langle L_{20-100 \mathrm{keV}}\right\rangle=\right.$ $\left.6.3 \times 10^{43} \mathrm{erg} \mathrm{s}^{-1}\right)$. The differences in luminosities are exactly the same when excluding the 5 Compton thick objects. The 16 blazars again appear brighter when assuming an isotropic emission, with $\left\langle L_{20-100 \mathrm{keV}}\right\rangle=10^{46} \mathrm{erg} \mathrm{s}^{-1}$. The latter value has to be used with caution: because the blazar emission is beamed towards the observer, not isotropic but collimated in a jet, blazars are highly variable and INTEGRAL detects them mainly in phases of outbursts.

\section{Discussion}

\subsection{The sample in comparison with previous studies}

For the whole population of sources seen by INTEGRAL we observe an increase in the fraction of unabsorbed objects compared to the first INTEGRAL AGN catalogue. Whereas $\frac{2}{3}$ of the Seyfert population in the first sample showed $N_{\mathrm{H}}>10^{22} \mathrm{~cm}^{-2}$, there are now more unabsorbed than absorbed sources, i.e. only $44 \%$ appear to be absorbed. A similar trend has also been observed in the Swift/BAT survey (Tueller et al. 2008), where the fraction is $\frac{1}{2}$. This trend is expected because with ongoing observations, IBIS/ISGRI and BAT perform deeper studies, which is also reflected in the increase in the average redshift and luminosity of the objects that are detectable. Sazonov et al. (2004, 2007) have shown that there is an anticorrelation of the fraction of absorbed sources with the X-ray luminosity. In a sample of 95 AGN detected by RXTE, they observed that the fraction 


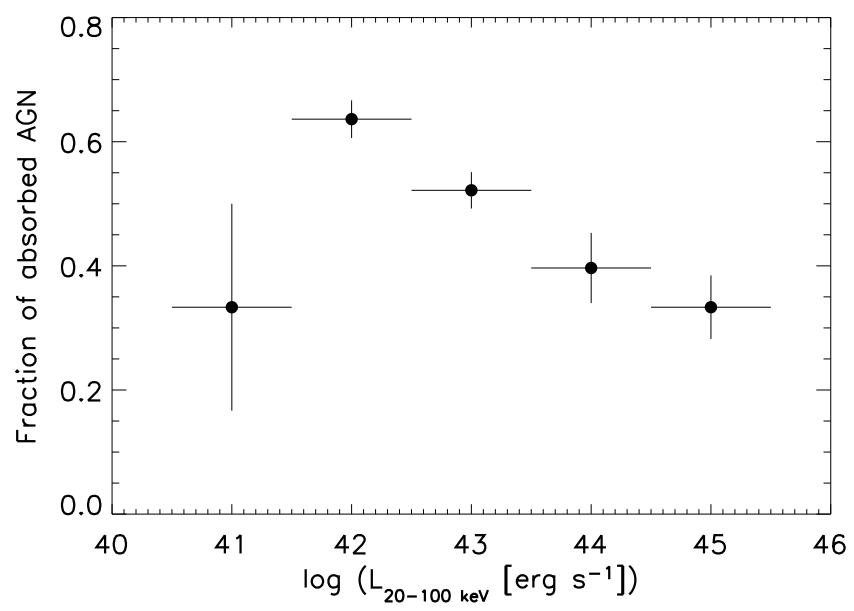

Fig. 7. Fraction of absorbed $\left(N_{\mathrm{H}}>10^{22} \mathrm{~cm}^{-2}\right)$ Seyfert galaxies as a function of hard X-ray luminosity for INTEGRAL detected AGN $(\geq 4 \sigma)$. The lowest luminosity bin includes only 3 sources.

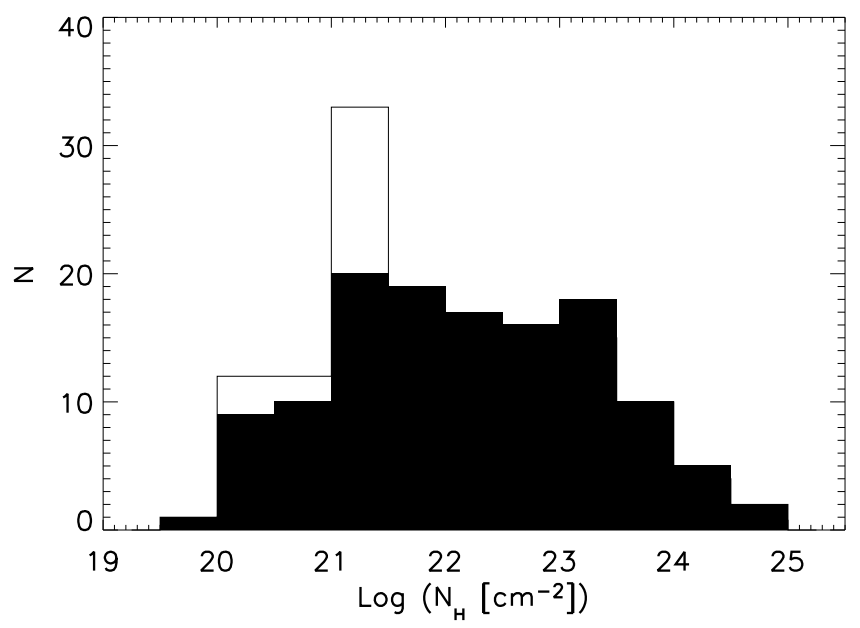

Fig. 8. Distribution of intrinsic absorption of the Seyfert content of INTEGRAL detected AGN $(\geq 4 \sigma)$. The unshaded area indicates objects where the $N_{\mathrm{H}}$ value is an upper limit.

of absorbed sources is $\sim 70 \%$ for $L_{3-20 \mathrm{keV}}<10^{43.5} \mathrm{erg} \mathrm{s}^{-1}$ but only $\sim 20 \%$ for objects with $L_{X}>10^{43.5} \mathrm{erg} \mathrm{s}^{-1}$. This trend can also be seen in the sample presented here. Determining the fraction of absorbed Seyfert galaxies in logarithmic bins of X-ray luminosity, we see the trend toward a decreasing fraction with luminosity in the range $L_{20-100 \mathrm{keV}}>3 \times 10^{41} \mathrm{erg} \mathrm{s}^{-1}$ (Fig. 7). The fraction appears low though in the lowest luminosity bin $\left(L_{20-100 \mathrm{keV}}<3 \times 10^{41} \mathrm{erg} \mathrm{s}^{-1}\right)$, but it has to be considered that this luminosity bin contains only 3 AGN, the Seyfert 1.5 galaxies NGC 4258, NGC 4395, and NGC 5033.

The fraction of Compton thick objects in the sample presented here is only $4 \%$ (5 objects out of the 135 Seyfert galaxies with measured intrinsic absorption). Although this sample is not a complete one, this indicates further that the fraction of Compton thick AGN is indeed $\ll 10 \%$ as already reported in e.g. Beckmann et al. (2006b) and Bassani et al. (2007). In addition, a recent study based on combined INTEGRAL and Swift/BAT data puts an upper limit of $\$ 9 \%$ on the fraction of Compton thick AGN (Treister et al. 2009), and one can thus consider most of the Seyfert population detected by INTEGRAL and Swift to be Compton thin. The distribution of intrinsic absorption (Fig. 8) shows that the Seyfert galaxies in the INTEGRAL sample are evenly distributed between $10^{21} \mathrm{~cm}^{-2}$ and $3 \times 10^{23} \mathrm{~cm}^{-2}$, as
Table 8. Correlation matrix for INTEGRAL AGN

\begin{tabular}{lccccc}
\hline \hline & $M_{\mathrm{BH}}$ & $\Gamma$ & $L_{\mathrm{X}}$ & $\begin{array}{c}\text { Edd. ratio } \\
\lambda\end{array}$ & $L_{V}$ \\
\hline$N_{\mathrm{H}}$ & no & no & no & no & no \\
$M_{\mathrm{BH}}$ & - & no & $>99.99 \%$ & intrinsic & $>99.99 \%$ \\
$\Gamma$ & & - & no & no & no \\
$L_{X}$ & & & - & intrinsic & $>99.99 \%$ \\
$\lambda$ & & & & - & no \\
\hline
\end{tabular}

already seen in the first INTEGRAL AGN catalogue (Beckmann et al. 2006c) and in the Swift/BAT AGN survey (Tueller et al. 2008). A significant amount of sources have reported $N_{\mathrm{H}}$ values of $N_{\mathrm{H}}<10^{21} \mathrm{~cm}^{-2}$. We assigned $N_{\mathrm{H}}=10^{21} \mathrm{~cm}^{-2}$ to these objects, which causes the peak in the distribution. The claim of two distinct groups resulting in two peaks in the $N_{\mathrm{H}}$ distribution as reported by Paltani et al. (2008) appears therefore caused by low number statistics. Although a clear dependency is seen on the intrinsic absorption when dividing the sample in two groups, there is no significant linear correlation with any of the parameters we tested. We summarize the analysis of the correlations in Table 8. Here the correlation probability is given if larger than $90 \%$. The correlations between Eddington ratio and $L_{\mathrm{X}}$ and $M_{\mathrm{BH}}$ are intrinsic, i.e. caused by the dependence of $\lambda$ on these two parameters.

For synthesis studies of the cosmic X-ray background one will have to take into account that both, the fraction of Compton thick and of absorbed sources, appears to be lower than assumed in most models published so far (e.g. Treister \& Urry 2005; Gilli et al. 2007). Although the average redshift in the INTEGRAL sample has increased significantly since the first catalogue from $\langle z\rangle=0.01$ to now $\langle z\rangle=0.03$, INTEGRAL will not be able to probe evolutionary effects. The only possibility of measuring evolutionary effects would be the combination of Swift/BAT data with a deep ( $10 \mathrm{Ms})$ INTEGRAL IBIS/ISGRI field (Paltani et al. 2008).

A systematic analysis of all BeppoSAX observations of AGN has been presented by Dadina (2007). Thirty-nine objects from this work are in common with the INTEGRAL sample. Four sources show strong differences in flux by more than a factor of 2 between the measurements, i.e. the Seyfert 2 galaxies NGC 4388, NGC 2110, NGC 7172, and the type 1 radio galaxy $3 \mathrm{C} 111$. All these objects are known to be variable at hardest X-rays (e.g. Beckmann et al. 2007). In addition, the average flux in the $20-100 \mathrm{keV}$ band appears to be higher in the INTEGRAL data than in the BeppoSAX one. This can be caused by the different time coverage: while BeppoSAX observations are mostly snapshots of the sources with duration of $\Delta t \ll 100 \mathrm{ks}$, the INTEGRAL observations of the AGN have the average exposure of $1200 \mathrm{ks}$. Thus the data presented here can include some short-term bright states, which would have been missed in most cases by BeppoSAX. In addition, the fluxes presented here are model fluxes, and because more complex modelling is necessary for the BeppoSAX spectra, applying a cut-off and a reflection component in most cases, this leads to lower broad-band model fluxes compared to the simple power law model applied here (see also Sect. 4.2). In addition, there is a systematic difference in the calibration of the ISGRI and PDS detectors, with steeper and higher-normalization Crab spectra extracted for ISGRI (Kirsch et al. 2005). This explains also larger spectral slope values fitted to the ISGRI spectra, when compared with the values quoted by Dadina (2007), although the primary reason for the these differences is the simpler spectral model applied to the INTEGRAL data. 
Table 9. OSSE detected AGN not seen by INTEGRAL.

\begin{tabular}{lcc}
\hline \hline Name & Type & $\begin{array}{c}\text { Exposure }^{a} \\
{[\mathrm{ks}]}\end{array}$ \\
\hline CTA 102 & blazar & 180 \\
H 1517+656 & BL Lac & 19 \\
III Zw2 & Sy1/2 & 15 \\
Mrk 279 & Sy1.5 & 11 \\
M82 & Starburst & 127 \\
NGC 253 & Starburst & 1 \\
NGC 7213 & Sy1.5 & 31 \\
NGC 7582 & Sy2 & - \\
PKS 2155-304 & BL Lac & 347 \\
QSO 1028+313 & Sy1.5 & 556 \\
\hline
\end{tabular}

${ }^{a}$ ISGRI exposure time.

CGRO/OSSE (Johnson et al. 1993) covered the energy range of approximately $50-10^{3} \mathrm{keV}$. It therefore primariliy detected AGN with hard and bright X-ray spectra, which we also expect to be detectable by INTEGRAL. While the first INTEGRAL AGN catalogue listed 24 OSSE-detected AGN not seen by INTEGRAL, this number has now decreased to 10 . We list those sources and the IBIS/ISGRI exposure time on the particular AGN in Table 9. It can be expected that the persistent sources in this list, i.e. the Seyfert and starburst galaxies, will be detected once a significant amount of exposure time is available for these objects.

As expected, only a few objects are jointly detected by INTEGRAL IBIS/ISGRI and Fermi/LAT, according to the bright source list based on three months of LAT data (Abdo et al. 2009). These 13 objects are blazars, except for the two radio galaxies Cen A and NGC 1275. The common blazars between ISGRI and LAT are 1ES 0033+595, PKS 0528+134, QSO B0716+714, Mrk 421, 3C 273, 3C 279, Mrk 501, PKS 1830-211, 1ES 1959+650, BL Lac, and 3C 454.3. The group of sources jointly detected by Fermi/LAT and ISGRI is expected to increase significantly through INTEGRAL target-ofopportunity (ToO) observations of blazars that show a flare in the LAT data.

\subsection{The intrinsic hard $X$-ray spectrum}

The effect that Seyfert 1 and low-absorbed objects appear to have steeper X-ray spectra than the Seyfert 2 and highly absorbed AGN was first noticed by Zdziarski et al. (1995), based on Ginga and CGRO/OSSE data and later confirmed e.g. by Gondek et al. (1996) using combined EXOSAT, Ginga, HEAO-1, and CGRO/OSSE spectra. A study of BeppoSAX PDS spectra of 45 Seyfert galaxies came to a similar conclusion, although the spectra of Seyfert 2 appeared steeper when considering a possible cut-off in the spectra of Seyfert 1 galaxies (Deluit \& Courvoisier 2003). The difference in the hard X-ray spectral slope between Seyfert 1 and 2 has been a point of discussion ever since its discovery. Zdziarski et al. (2000) considered the anisotropy of Compton scattering in planar geometry and effects of reflection, but came to the conclusion that this cannot be the sole explanation. Beckmann et al. (2006) argued that the difference might be a selection effect, as objects have to have a harder $\mathrm{X}$-ray spectrum to be detectable when strong intrinsic absorption is present. With the growing sample of AGN, this argument does not seem to hold, as the absorption in the energy band $>20 \mathrm{keV}$ is negligible for Compton thin objects, and also the ongoing identification effort of newly detected hard X-ray sources did not reveal a different population than already presented in Beckmann et al. (2006).

A solution might be provided when considering the effects of Compton reflection on the hard X-ray spectrum, as shown in the previous section. Recent analysis of a sample of 105 Seyfert galaxies using the spectra collected with BeppoSAX in the 2-200 keV band (Dadina 2008) provided no evidence of any spectral slope difference when applying more complex model fitting including a reflection component (PEXRAV). The mean photon index values found for Seyfert 1 and Seyfert 2 samples were $\Gamma=1.89 \pm 0.03$ and $\Gamma=1.80 \pm 0.05$. The difference between types 1 and 2 is seen in this model in the different strength of the reflection component, with $R=1.2 \pm 0.1$ and $R=0.9 \pm$ 0.1 , and different cut-off energies of $E_{\mathrm{C}}=230 \pm 22 \mathrm{keV}$ and $E_{\mathrm{C}}=376 \pm 42 \mathrm{keV}$, for Seyfert 1 and Seyfert 2, respectively. It has to be pointed out that spectral slope, reflection strength, and cut-off energy are closely linked. The IBIS/ISGRI data have a disadvantage over broad-band data when studying the spectral shape of the hard X-ray continuum, as we lack information about the spectrum below $18 \mathrm{keV}$. But on the positive side, using data from only one instrument, the spectra do not suffer from the problem of intercalibration factors, which is apparent in all studies using different instruments, and especially when using data from different epochs. In those cases, where spectra are taken by more than one instrument at different times, the flux variability can mimic a stronger or weaker reflection component or cut-off energy (e.g. Panessa et al. 2008).

The INTEGRAL data show consistent slopes for the spectra of unabsorbed/type 1 and absorbed/type 2 objects already when a simple power-law model is used. When applying the model used by Dadina (2007) to the stacked INTEGRAL spectrum of Seyfert galaxies, we get similar results: the underlying powerlaw appears to have consistent (within $2 \sigma$ ) spectral slope for type $1(\Gamma=1.96)$ and type $2(\Gamma=1.91)$ objects and the same reflection strength $R \simeq 1$.1. The data do not allow to determine the cut-off energy or inclination angle when fitting the reflection component (Fig. 6). When fitting a simple cut-off power law, the INTEGRAL data show the same trend as the BeppoSAX sample, i.e. a lower cut-off energy for Seyfert $1\left(E_{\mathrm{C}}=86 \mathrm{keV}\right)$ than for Seyfert $2\left(E_{\mathrm{C}}=184 \mathrm{keV}\right)$. It has to be taken into account, though, that the fit to the Seyfert 2 data is bad quality, and that fixing the cut-off here to the same value as derived for the Seyfert 1, also leads to the same spectral slope. When fitting a reflection model to the stacked data, one gets a consistent photon index of $\Gamma \simeq 1.95$ and reflection strength $R \simeq 1.3$ for both absorbed and unabsorbed AGN. These values of $\Gamma$ and $R$ agree with the correlation $R=(4.54 \pm 1.15) \times \Gamma-(7.41 \pm 4.51)$ Dadina (2008) found for the BeppoSAX AGN sample, which, for the INTEGRAL sample with $\Gamma=1.95$, would lead to $R=1.4$. This $R(\Gamma)$ correlation was first noted based on Ginga data for extragalactic and Galactic black holes, leading to $R=(1.4 \pm 1.2) \times$ $10^{-4} \Gamma^{(12.4 \pm 1.2)}$ (Zdziarski et al. 1999), which in our case would result in a smaller expected reflection component with $R=0.6$ but within $1 \sigma$ of the value detected here. Absorbed and unabsorbed show a consistent turnover at about $E_{\mathrm{C}}=100 \mathrm{keV}$ when a cut-off power law model is applied.

The observed dichotomy of different spectral slopes for type 1 and type 2 objects might therefore be caused by data with too low significance, which do not allow to fit the reflection component, or in general by a strong dependence of the spectral slope on the choice of the fitted model. One aspect that has to be kept in mind is the dependence of the reflection strength $R$ on the model applied and on the geometry assumed. Murphy \& Yaqoob (2009) showed recently that their model of a reflection 


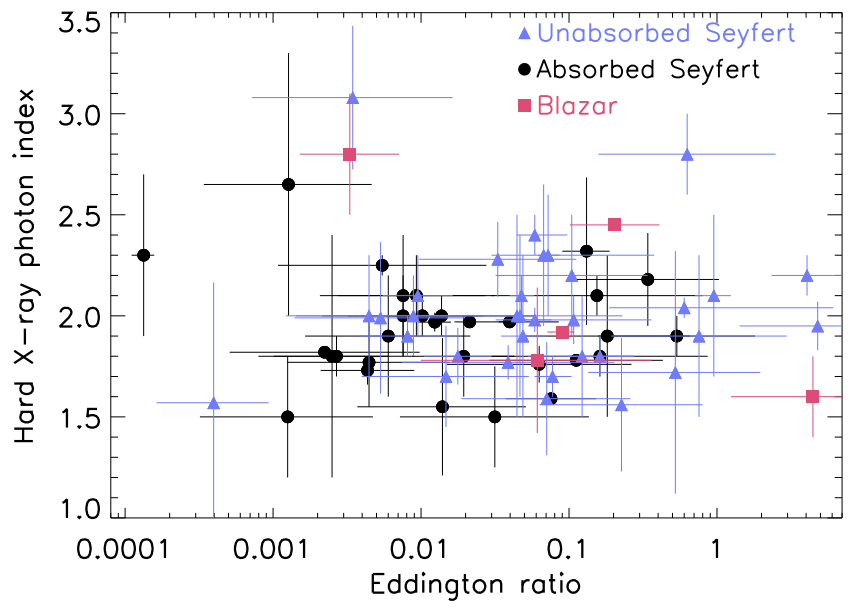

Fig. 9. Photon index of a simple power-law fit to the IBIS/ISGRI data versus Eddington ratio $\lambda$.

spectrum from a Compton-thick face-on torus that subtends a solid angle of $2 \pi$ at the X-ray source is a factor of $\sim 6$ weaker than that expected from a Compton-thick, face-on disc as modelled in PEXRAV. Therefore, applying a torus model to the data presented here would result in much less reflection strength.

\subsection{Eddington ratios and accretion rates in Seyfert galaxies}

A different approach to search for differences or similarities in Seyfert galaxies is to study the accretion rates. Middleton et al. (2008) suggest that different accretion states lead to differences in the hardness of hard X-ray $(E>10 \mathrm{keV})$ spectra between types 1 and 2 AGN (type 1 spectra being systematically softer). Using data from CGRO/OSSE, BeppoSAX, and INTEGRAL, they found that the 24 Seyfert 2 galaxies in their sample of hard X-ray selected AGN show an accretion rate (parameterized with the Eddington ratio) in average smaller than that of their 23 Seyfert 1 . This effect has also been seen when studying AGN detected by Swift/BAT (Winter et al. 2009). This would be consistent with all accreting black holes in general showing harder spectra at low accretion rates (Laor 2000; Remillard \& McClintock 2006).

The 20-100 keV luminosities as derived from the IBIS/ISGRI data were used to approximate the bolometric luminosity. Assuming a canonical photon index of 2.0 for a single power law, the total X-ray luminosity is about $L_{(1-200 \mathrm{keV})}=$ $3 \times L_{(20-100 \mathrm{keV})}$. Assuming that the first peak of the spectral energy distribution is as strong as the X-ray luminosity, we derive $L_{\mathrm{Bol}}=6 \times L_{(20-100 \mathrm{keV})}$. The Eddington luminosity considering pure hydrogen is given by $L_{\mathrm{Edd}}=1.26 \times$ $10^{38} \frac{M}{M_{\odot}} \mathrm{erg} \mathrm{s}^{-1}$, and thus the Eddington ratio is $\lambda=L_{\mathrm{Bol}} / L_{\mathrm{Edd}}=$ $4.8 \times 10^{-38} L_{(20-100 \mathrm{keV})} \frac{M_{\odot}}{M} \mathrm{erg}^{-1} \mathrm{~s}$. The Eddington ratio can be computed for 71 Seyfert objects of the sample presented here. The photon index as determined from IBIS/ISGRI data and the Eddington ratio are compared in Fig. 9. The four AGN on the top left of this figure, with steep spectra and low Eddington ratio $(\lambda<0.005)$, are the Seyfert 1 1H 1934-063, the Seyfert 2 objects SWIFT J0601.9-8636 and NGC 4258, and the blazar Mrk 501. There is no significant correlation detectable, even when excluding these outliers. As observed by Steffen et al. (2003) in Chandra data of AGN, it appears that the 2-8 keV luminosity function is dominated by type $1 \mathrm{AGN}$ at high X-ray luminosities and by type 2 at low luminosities. The same effect is seen

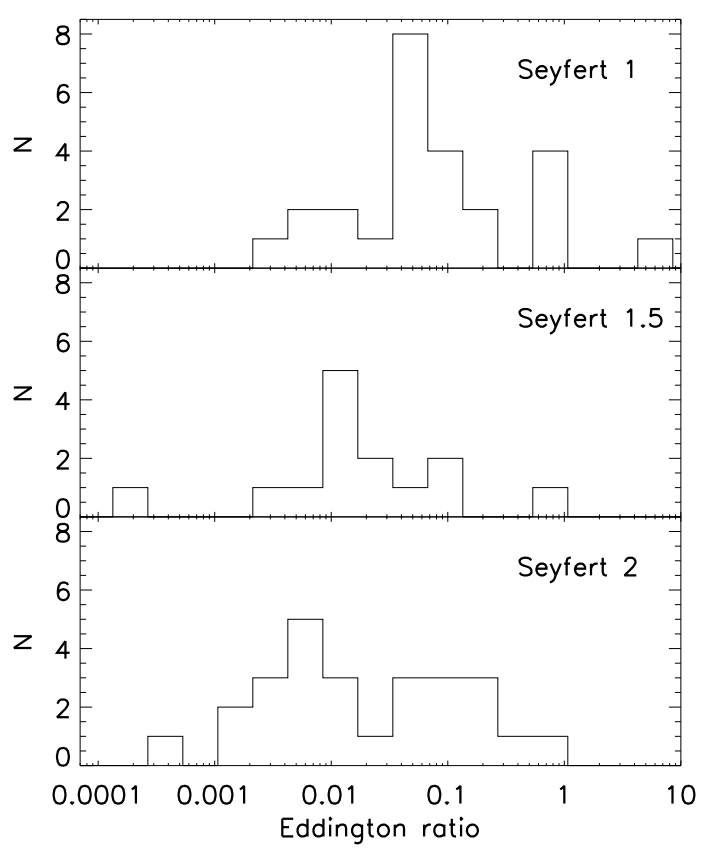

Fig. 10. Distribution of Eddington ratios for Seyfert 1, 1.5, and type 2 AGN in the sample.

also in the INTEGRAL luminosity function (Beckmann et al. 2006b). Connected to this, we observe not only that the absorbed sources are less luminous than the unabsorbed ones, but also that absorbed sources have smaller accretion rates as seen in lower Eddington ratios (Fig. 10). In agreement with Middleton et al. (2008) results, we find that the average values of Eddington ratio for Seyfert $1\left(\left\langle\lambda_{\text {Sy1 }}\right\rangle=0.064\right)$ are higher than those found for intermediate Seyfert type $\left(\left\langle\lambda_{\text {Sy1.5 }}\right\rangle=0.015\right)$ and Seyfert 2 with $\left\langle\lambda_{\text {Sy } 2}\right\rangle=0.02$ (Fig. 10), although we do not observe the differences in the underlying spectra, as seen in their study. The same applies for the separation into unabsorbed $\left(\left\langle\lambda_{\left(N_{\mathrm{H}}<10^{22} \mathrm{~cm}^{-2}\right)}\right\rangle=\right.$ $0.06)$ and absorbed sources $\left(\left\langle\lambda_{\left(N_{\mathrm{H}}>10^{22} \mathrm{~cm}^{-2}\right)}\right\rangle=0.015\right)$. To calculate the probability that the Eddington ratios of Sey $1 /$ unabsorbed objects and Sey2/absorbed AGN are drawn from the same population, we applied a Kolmogorov-Smirnov test. We can reject the null hypothesis (same population), with a probability of false rejection of $0.1 \%$ and $3 \%$ for the Seyfert 1 - Seyfert 2 and unabsorbed - absorbed objects, respectively. On the other hand, since we do not find a significant correlation between the hard $\mathrm{X}$-ray photon index and the Eddington ratio, we cannot back up the scenario by Middleton et al. (2008) as a way to explain the different spectral hardness of type 1 and type 2 AGN.

When studying the effect of radiation pressure on dusty absorbing gas around AGN, Fabian et al. (2008) trace a region in the $N_{\mathrm{H}}$-Eddington ratio plane that is forbidden to long-lived clouds in AGN. In fact, even when the AGN is in the subEddington regime for the ionized gas, it can appear to be superEddington for the dusty gas, hence ejecting the surrounding absorbing clouds. Objects in this region of the $N_{\mathrm{H}}$-Eddington ratio plane could present outflows or show transient or variable absorption. Fabian et al. (2009) tested the predictions of this model using the Swift/BAT AGN sample of Winter et al. (2008) and find a good agreement, with only 1 object lying in the forbidden area, the Seyfert 1.9 object MCG-05-23-016. This object has been shown to exhibit high accretion rates at the Eddington limit (Beckmann et al. 2008). In Fig. 11 we show the regions defined by Fabian et al. (2009) in the $N_{\mathrm{H}}$-Eddington ratio plane and our AGN sample. The forbidden region is on the 


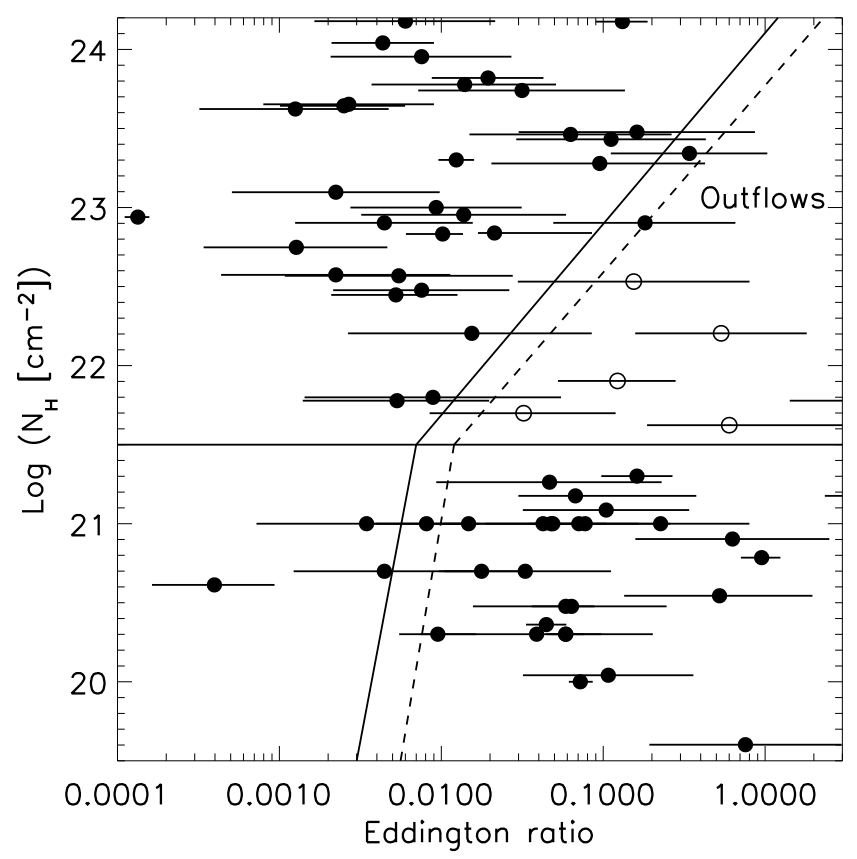

Fig. 11. Intrinsic absorption versus Eddington ratio for the Seyfert galaxies in the INTEGRAL sample. The "forbidden zone" described by Fabian et al. (2009) is occupied by 5 INTEGRAL AGN (empty circles).

upper-righthand side of the plot and bordered by an upper limit to $N_{\mathrm{H}}$ (for absorption due to dust lanes at few kpc from the AGN centre) and the Eddington limit for the dusty gas when only the black hole mass is considered to be gravitationally important (continuous line) or if also as much mass from intervening stars is included (dashed line). It is important to keep in mind that the bolometric luminosity is a critical parameter in defining the limits of the forbidden area, as different estimates of it can be used. Therefore, using a bolometric correction by a factor of few larger than what we applied here would shift the data points towards higher Eddington ratios, occupying the forbidden region completely. Nevertheless, with the bolometric luminosity we estimate $\left(L_{\mathrm{Bol}}=6 \times L_{(20-100 \mathrm{keV})}\right)$, and find 5 objects with a detection significance $>4 \sigma$ in this area: IGR J00335+6126, MCG-05-23-016, Mrk 766, IC 4329A, and NGC 5506. Out of these, only MCG-05-23-016 occupies the forbidden region also in Fabian et al. (2009), whereas IGR J00335+6126 was not included and Mrk 766 lacked absorption information in their study, IC 4329A is located close to the border of the forbidden zone, and NGC 5506 is not within this area. This raises the question of whether this region indeed cannot be occupied persistently by Seyfert type AGN, although the 5 objects do display some peculiarities, as described in the following. IC 4329A and IGR J00335+6126 are just above the upper limit $N_{\mathrm{H}}$ and MCG-05-23-016 has a complex spectrum with warm absorbers, not necessarily related to dusty gas (Fabian et al. 2009). The Seyfert 1.9 NGC 5506 has been identified as an obscured narrow-line Seyfert 1 (Nagar et al. 2002), which might explain the high accretion rate in this object. And the Seyfert 1.5 Mrk 766 with a black hole mass of only $3.5 \times 10^{6} M_{\odot}$ (Uttley \& McHardy 2005) might be a similar case to the highly efficient Seyfert MCG-05-23-016 (Beckmann et al. 2008). But the small number of objects and the fact that the uncertainty on the Eddington ratio is still rather large do not allow us to conclusively state that strongly absorbed Seyfert galaxies cannot exhibit high accretion rates over a long phase of their lifetimes.

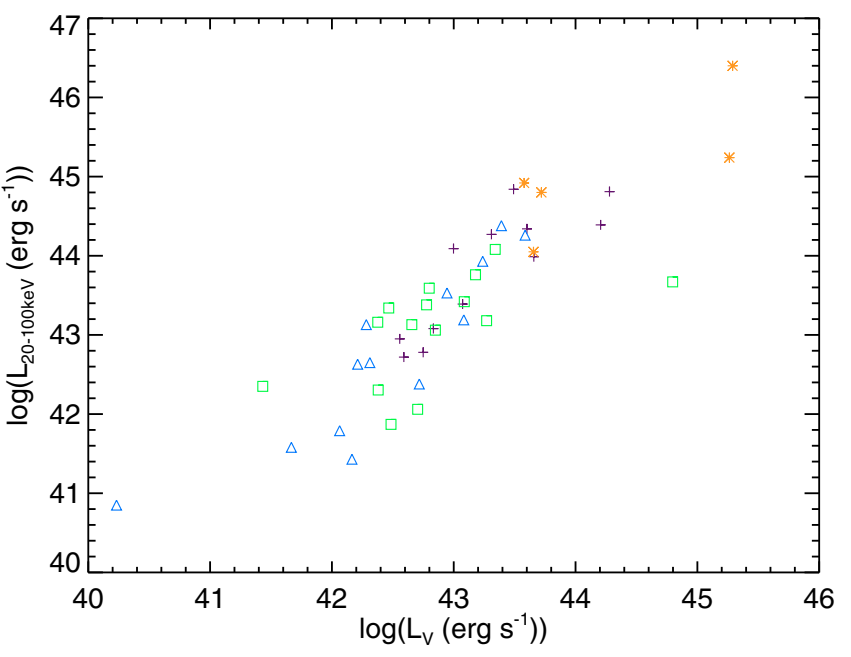

Fig. 12. X-ray luminosity $L_{\mathrm{X}}$ versus $V$-band luminosity $L_{V}$. AGN of different types are located along the same dispersion line, over more than 5 decades in luminosity. Symbols: crosses - Seyfert 1-1.2; triangles Seyfert 1.5; squares - Seyfert 1.8-2; asterisks - BL Lacs.

\subsection{Correlations with optical data and $\alpha_{O X}$}

We show in Fig. 12 the dispersion diagram of $L_{\mathrm{X}}$ vs. $L_{V}$, with different symbols for the different classes of objects considered. While the apparent correlation between both luminosities is certainly driven by the distance effect evident in Fig. 5, it is remarkable that the different classes of objects, from Seyfert 2 to blazars, are located on the same correlation line, over more than 5 decades in luminosity. Subtracting the common dependence on redshift through a partial correlation analysis, the correlation between the luminosities is still statistically significant, with a correlation coefficient of 0.74 and the probability of a chance occurrence is $\ll 0.01 \%$. To test this correlation further, we made simulations using a bootstrap method (Simpson et al. 1986): to each couple of X-ray flux and redshift we randomly assigned an optical flux, drawn from the real values found for our sample and without excluding multiple choices of the same value (see also Bianchi et al. 2009, for a similar procedure). We then computed the X-ray and optical luminosities and calculated the Spearman and the partial correlation coefficients for the $L_{\mathrm{X}}-L_{V}$ relation and the Spearman coefficient for the $F_{\mathrm{X}}-F_{V}$ relation. Repeating this procedure 100000 times, we were able to build histograms of the correlation coefficients of the simulated samples and found that only $0.001 \%$ of the simulated samples have a (Spearman or partial) correlation coefficient greater than measured in the real sample for the $L_{\mathrm{X}}-L_{V}$ relation. For the $F_{\mathrm{X}}-F_{V}$ correlation, the probability of chance occurrence is higher but still not significant, $\sim 2 \%$. This indicates that the $\mathrm{X}$-ray and optical emissions are indeed correlated, beyond the bias introduced by the common dependence on distance.

To further investigate this relation, we computed the histograms of the $\alpha_{\text {OX }}$ values for the different subtypes. We show in Fig. 13 that the peaks of the distributions coincide for the four subsamples, with mean values close to each other (Seyfert 2: 1.14, Seyfert 1.5: 1.13, Seyfert 1-1.2: 1.08, and BL Lac: 1.03). Seyfert 2 and 1.5 nevertheless show an extended wing towards higher $\alpha_{\mathrm{OX}}$ ratios. NGC 1068, the prototypical Seyfert 2 galaxy, shows the highest $\alpha_{\mathrm{OX}}$ value, 1.38. Because it is a Comptonthick object, this high $\alpha_{\mathrm{OX}}$ value can be understood clearly. Other Compton-thick Seyfert galaxies, such as NGC 3165 and ESO138-1, also show $\alpha_{\text {OX }}$ values above 1.20. But other objects with high $\alpha_{\text {OX }}$ values, such as NGC 1052 and NGC 5033, 


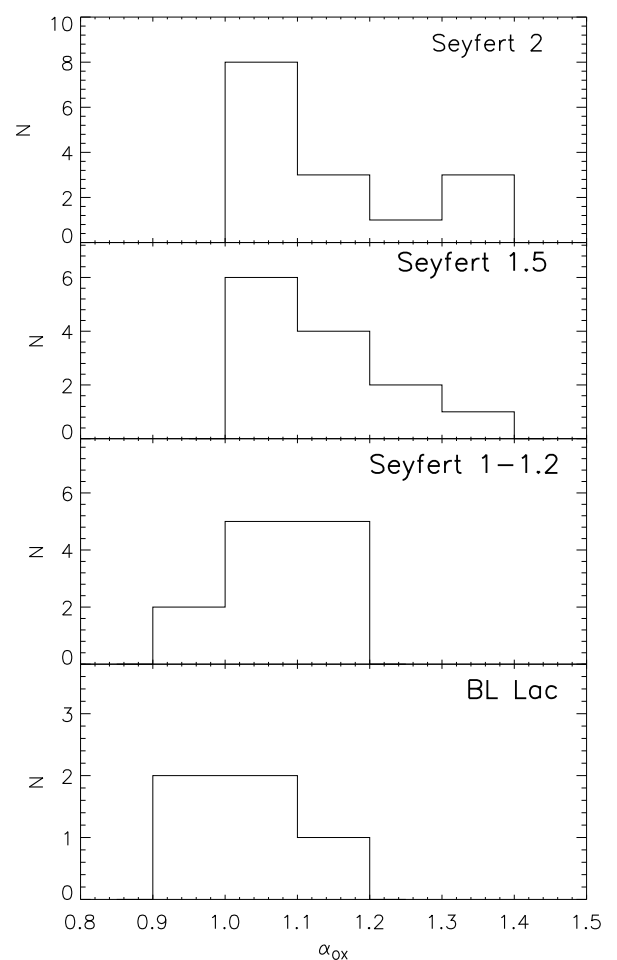

Fig. 13. $\alpha_{\mathrm{OX}}$ histograms for 4 subtypes of AGN.

have low hydrogen column densities; in contrast several objects with high neutral columns, like NGC 3281, NGC 4945, and ESO 383-18, show relatively low $\alpha_{\text {OX }}$ values.

As discussed in Sect. 2.3.1, only 3 objects in the sample have shown significant optical variability over the period being monitored $(\Delta V>0.5 \mathrm{mag})$. For the blazars QSO B0716+714 and 3C 279 , both their $L_{V}$ and $L_{X}$ values have to be dominated by the central AGN, with only a minor contribution by stars in $V$. This is indeed expected for this type of high-luminosity blazars while accretion processes and bulge stars dominate the $V$ band in less active Seyfert galaxies.

That most Seyfert galaxies in the sample look similar when analysed from the point of view of their $\alpha_{\mathrm{OX}}$ value indicates that the true nature of these objects is indeed very similar, thus supporting the unified scenario of AGN. The central black hole behaves very similarly with respect to the host galaxy, independent of the type of object. The classification as type 1 or type 2 AGN would mainly derive from observations of parameters dependent on geometrical effects, such as the profile of the emission lines or of the X-ray emission, but would not be tracing systematic differences in the intrinsic nature of these objects.

\subsection{A fundamental plane of AGN activity}

In the view of unification of different AGN types, it has been pointed out that AGN, spanning black hole masses in the range of $10^{5} M_{\odot} \lesssim M_{\mathrm{BH}} \lesssim 10^{9} M_{\odot}$ and even accreting black holes in X-ray binaries with $M_{\mathrm{BH}} \sim 10 M_{\odot}$, show similarities in radiative efficiency and jet power versus accretion rate (e.g. Fender et al. 2007). This connection gave rise to the "fundamental plane" of black hole activity. It has been found that indeed there is a close connection between the radio and X-ray luminosity of Galactic and super massive black holes of the form $L_{\text {radio }} \propto L_{\mathrm{X}}^{0.7}$ (Corbel et al. 2000, 2003), linking the jet activity to the total output of the central engine. Later on, a connection of these two

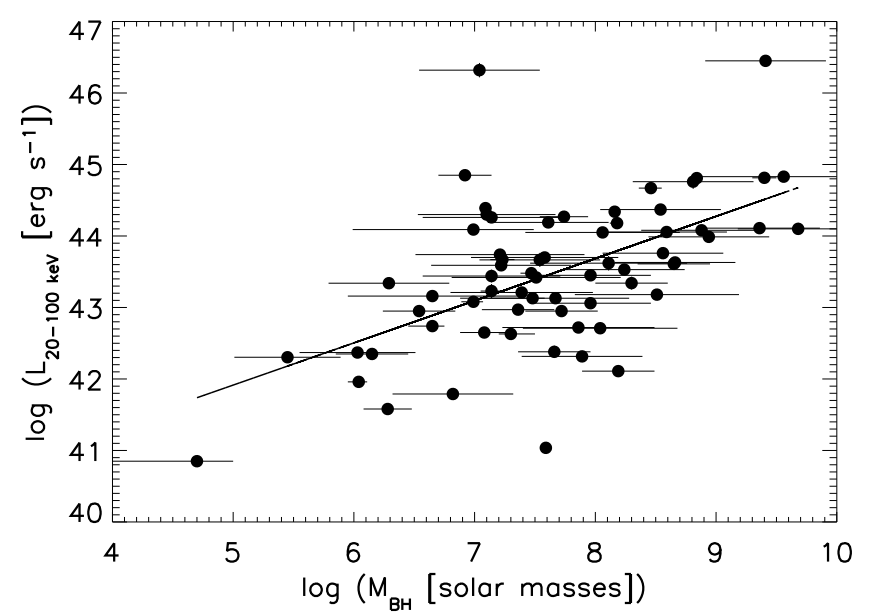

Fig. 14. Hard X-ray luminosity versus black hole masses for Seyfert galaxies. Errors on $L_{X}$ are smaller than the symbols. More massive AGN appear to be brighter in the hard X-rays with $L_{\mathrm{X}} \sim M_{\mathrm{BH}}^{0.7 \pm 0.1}$. The correlation coefficient is 0.52 with a probability of non-correlation $<2 \times 10^{-6}$.

parameters with the black hole mass itself was found, establishing the fundamental plane of AGN and Galactic black hole activity in the form of $L_{\text {radio }} \propto L_{\mathrm{X}}^{0.6} M_{\mathrm{BH}}^{0.8}$ (e.g. Merloni et al. 2003).

The correlation between X-ray luminosity and black hole mass is also common to all objects in our sample, showing indeed that more massive AGN are more luminous $\left(L_{\mathrm{X}} \sim M_{\mathrm{BH}}^{0.7 \pm 0.1}\right.$, as shown in Fig. 14). A similar relation has lately been reported for various X-ray selected AGN samples (e.g. Bianchi et al. 2009; Wang et al. 2009), and also in these cases the slope is lower than 1 . This could indicate that more massive black holes either have lower accretion rates than less massive objects, or a smaller fraction of their total power is converted into X-ray luminosity (assuming that the bolometric luminosity scales linearly with black hole mass). As reported in Table 8, a significant correlation is also detected between the black hole mass and the optical luminosity. Only for 8 out of 41 objects might the correlation of $L_{V}$ versus $M_{\mathrm{BH}}$ be induced by the method used to estimate the mass, i.e. when deriving the mass from the K-band magnitudes (assumed to represent the bulge luminosity) or from continuum optical luminosity. However, caution should be used in general when considering a correlation between luminosity and black hole mass, because possible selection effects or different biases could contribute to the observed correlation in non complete samples. Woo \& Urry (2002) argue that, when correlating bolometric luminosity with black hole mass, the Eddington luminosity sets a (soft) upper limit to the luminosities and therefore determines the empty region of the diagram in the upper left corner. On the other hand, the lower-right corner should be populated by low-luminosity, massive black holes that are not included or are rare in the high-energy AGN samples, such as normal or radio galaxies.

As discussed in detail in the previous section, there is also a significant correlation between optical and X-ray luminosity. Therefore, as summarised in Table 8, we found three significant correlations in our sample between the luminosities $L_{\mathrm{X}}$ and $L_{V}$, and the mass of the central black hole $M_{\mathrm{BH}}$. This leads to the assumption that also these parameters, similar to $L_{\mathrm{X}}, L_{R}$, and $M_{\mathrm{BH}}$, form a fundamental plane for AGN. By applying an analysis following Merloni et al. (2003), we fit the data with the function

$\log L_{V}=\zeta_{V \mathrm{X}} \log L_{\mathrm{X}}+\zeta_{V M} \log M_{\mathrm{BH}}+b_{V}$ 


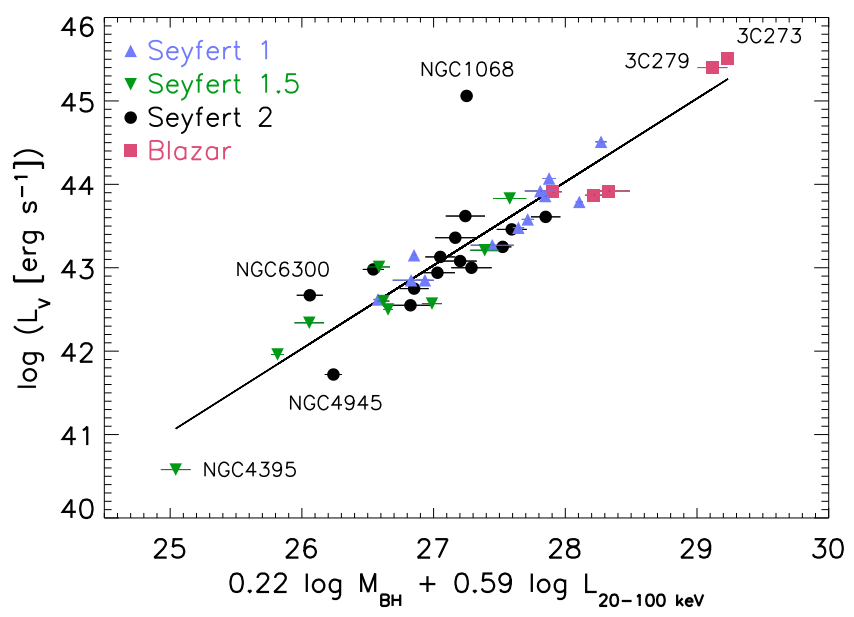

Fig. 15. Fundamental plane of optical luminosity $L_{V}$, X-ray luminosity $L_{\mathrm{X}}$, and mass of the central black hole $M_{\mathrm{BH}}$. Errors on luminosity are smaller than the symbols.

We obtain $\zeta_{V X}=0.59 \pm 0.07, \zeta_{V M}=0.22 \pm 0.08$, and $b_{V}=16.0$, leading to

$\log L_{V}=0.59 \log L_{\mathrm{X}}+0.22 \log M_{\mathrm{BH}}+16.0 ;$

i.e., $L_{V} \propto L_{\mathrm{X}}^{0.6} M_{\mathrm{BH}}^{0.2}$. We show this relation in Fig. 15 . As already pointed out and discussed here and in Sect. 4.4, we carefully investigated that the effects of distance and selection effect are not causing the correlation observed here.

While the fundamental plane including the radio and X-ray luminosity can be understood as a connection of the jet activity, as visible in the radio, and the accretion flow, as dominating the $\mathrm{X}$-rays, the correlation found here shows a different connection. The optical luminosity is commonly thought to be dominated by the AGN accretion disc (e.g. Siemiginowska et al. 1995) and therefore by the accretion processes onto the supermassive black hole, but there is also a possible contribution by the jet (Soldi et al. 2008) and emission of the bulge, and therefore of the stars in the host galaxy contributing to it. The latter is especially important as the resolution of INTEGRAL's OMC camera does not allow a deconvolution of the core and the bulge. Nevertheless it shows that there is a significant bulge- $M_{\mathrm{BH}}$ correlation and that accretion processes are closely linked to the mass of the central black hole. The finding that this fundamental plane holds for all Seyfert types indicates further that these AGN are indeed intrinsically the same.

\section{Conclusions}

We have presented the second INTEGRAL AGN catalogue, including 187 extragalactic objects. The AGN population detected by INTEGRAL is dominated by Seyfert galaxies in the local $(\langle z\rangle=0.03)$ universe, with moderate X-ray luminosity $\left(\left\langle L_{20-100 \mathrm{keV}}\right\rangle=4 \times 10^{43} \mathrm{erg} \mathrm{s}^{-1}\right)$. Seyfert 1 galaxies appear to have higher luminosities $\left(\left\langle L_{20-100 \mathrm{keV}}\right\rangle=10^{44} \mathrm{erg} \mathrm{s}^{-1}\right)$ and Eddington ratio $\left(\left\langle\lambda_{\mathrm{Sy} 1}\right\rangle=0.064\right)$ than the Seyfert 2 galaxies $\left(\left\langle L_{20-100 \mathrm{keV}}\right\rangle=2.5 \times 10^{43} \mathrm{erg} \mathrm{s}^{-1},\left\langle\lambda_{\mathrm{Sy} 2}\right\rangle=0.02\right)$. Although IBIS/ISGRI spectra alone lack the information about the iron line complex and the continuum shape below $18 \mathrm{keV}$, they can be used to study the average Seyfert spectra in a statistical way. The underlying continuum of the hard X-ray spectrum appears to be consistent between different Seyfert types, both when a simple power-law model is applied and when considering the effects of Compton reflection. Applying the PEXRAV reflection model with no high energy cut-off, the Seyfert 1 and 2 galaxies show the same underlying power law with $\Gamma \simeq 1.95$ and a reflection component of $R \simeq 1.1$, when applying different inclination angles of $i \simeq 30^{\circ}$ and $i \simeq 60^{\circ}$, respectively. Although, when applying a cut-off power law model to the stacked spectra, the Seyfert 1 show lower cut-off energies $\left(E_{\mathrm{C}}=86_{-14}^{+21} \mathrm{keV}\right)$ than the Seyfert 2 objects $\left(E_{\mathrm{C}}=184_{-52}^{+16} \mathrm{keV}\right)$, the bad quality of the fit in the latter case and that fixing the cut-off to the value of the Seyfert 1 leads to a similar spectral slope might indicate that the spectra are intrinsically indeed the same.

The same differences as for different Seyfert classes are observable when considering the intrinsic absorption: the unabsorbed sources also have higher luminosities $\left(\left\langle L_{20-100 \mathrm{keV}}\right\rangle=\right.$ $\left.6.3 \times 10^{43} \mathrm{erg} \mathrm{s}^{-1}\right)$ and Eddington ratio $(\langle\lambda\rangle=0.06)$ than the absorbed AGN $\left(\left\langle L_{20-100 \mathrm{keV}}\right\rangle=2.5 \times 10^{43} \mathrm{erg} \mathrm{s}^{-1},\langle\lambda\rangle=0.015\right)$. Also separating the objects into absorption classes, the underlying continuum appears similar when considering the effects of Compton reflection. The mass of the central black hole is on average the same among the different Seyfert types and absorption classes, with $\left\langle M_{\mathrm{BH}}\right\rangle=4 \times 10^{7} M_{\odot}$. Also comparing optical to hard X-ray emission, the different Seyfert classes show the same ratio $\left(\alpha_{\mathrm{OX}}=1.1\right)$. On average, the hard X-ray spectra of Seyfert 1.5 objects are closer to those of the Seyfert 1 class than to Seyfert 2.

The optical data provided by INTEGRAL/OMC can be used to monitor variability. Strong variability $(\Delta V \gtrsim 0.5 \mathrm{mag})$ is only seen in three objects within the optical sample of $57 \mathrm{AGN}$, i.e. in the blazars QSO B0716+714 and 3C 279, and in NGC 4151.

The overall picture can be interpreted within the scenario of a unified model. The whole hard X-ray detected Seyfert population fills the parameter space of spectral shape, luminosity, and accretion rate smoothly, and only an overall tendency is seen in which more massive objects are more luminous, less absorbed, and accreting at higher Eddington ratio. An explanation for why the absorbed sources have been claimed to show flatter spectra in the hard X-ray domain when fit by a simple power law can be that the slope of the continuum strongly depends on the fitted model and that Compton reflection processes play a major role here. Considering these effects, it appears that the different Seyfert types are indeed intrinsically the same.

More evidence for the unified scheme is that a fundamental plane can be found between the mass of the central object and optical and X-ray luminosity. The correlation takes the form $L_{V} \propto L_{\mathrm{X}}^{0.6} M_{\mathrm{BH}}^{0.2}$, similar to what is found in previous studies between $L_{R}, L_{\mathrm{X}}$, and $M_{\mathrm{BH}}$. This links the accretion mechanism with the bulge of the host galaxy and with the mass of the central engine in the same way in all types of Seyfert galaxies. The connection is also apparent through the same optical-to-hard X-ray ratio measured in all Seyfert classes.

Evolutionary effects are likely to be beyond the AGN population accessible by INTEGRAL and Swift. Deep hard X-ray surveys by future missions like NuSTAR, Astro-H, and EXIST will be able to answer this question through deep observations of small portions of the sky.

Acknowledgements. INTEGRAL is an ESA project funded by ESA member states (especially the PI countries: Denmark, France, Germany, Italy, Spain, Switzerland), Czech Republic, Poland, and with the participation of Russia and the USA. We thank the anonymous referee for the comments that helped to improve the paper. This research has made use of data obtained through the High Energy Astrophysics Science Archive Research Center Online Service, provided by the NASA/Goddard Space Flight Center. We acknowledge the use of public data from the Swift data archive and from the INTEGRAL data archive provided by the ISDC. S.S. acknowledges the support by the Centre National d'Etudes Spatiales (CNES). P.L. and A.A.Z. have been supported in part by the Polish MNiSW grants NN203065933 and 362/1/N-INTEGRAL/2008/09/0, and 
the Polish Astroparticle Network 621/E-78/BWSN-0068/2008. J.M.M.H., A.D. and J.A. are supported by the Spanish MICINN grant ESP2008-03467.

\section{Appendix A: Notes on individual sources}

We include here all Seyfert galaxies above $30 \sigma$ IBIS/ISGRI detection significance, all sources showing a complex ISGRI spectrum (Table 3), and those for which the results found here differ from previous works.

Mrk 348: the X-ray spectrum of this Seyfert 2 was studied by RXTE, showing the same spectral shape as reported here ( $\Gamma=1.8$ ) and evidence for a reflection component with $R \lesssim 1$ (Smith et al. 2001). Instead of a cut-off power law with $\Gamma=0.9$ and $E_{\mathrm{C}}=55 \mathrm{keV}$ as given in Table 3, the INTEGRAL data can be equally well represented by a Compton reflection model (PEXRAV) with $R=1$ and photon index $\Gamma=1.8$ and no highenergy cut-off.

NGC 1275 presents a very complex spectrum in INTEGRAL data as it includes several components of different physical origin. While the hard X-ray spectrum visible in the IBIS/ISGRI data is dominated by the narrow-line radio galaxy NGC 1275 and its spectrum above $20 \mathrm{keV}$ can be represented by a simple power law model, we observe in JEM-X the Perseus galaxy cluster. An extensive discussion of the INTEGRAL spectrum has been presented in Eckert \& Paltani (2009).

NGC 4051: this Seyfert 1.5 shows a strong reflection component when fit together with soft X-rays, e.g. $R \simeq 7$ for $S u z a k u$ (Terashima et al. 2009), and $R \simeq 6$ for combined Swift/XRT and IBIS/ISGRI data (Beckmann et al. 2009), while the data presented here allow only to fit a single power law model with $\Gamma=2.1 \pm 0.2$. When fitting the Suzaku data with a simple power law model, Terashima et al. (2009) derive a photon index of $\Gamma=1.5_{-0.2}^{+0.3}$ for a low flux state, indicating strong flux and spectral variability.

NGC 4151: this bright AGN allows complex modelling beyond the scope of this paper, and we refer to an early INTEGRAL analysis by Beckmann et al. (2005), to an analysis of BeppoSAX data by de Rosa et al. (2007), and to a study of the different spectral states by Lubiński et al. (2009).

NGC 4388: the hard X-ray data of this Seyfert 2 galaxy have been studied by Beckmann et al. (2004). Their analysis of INTEGRAL, XMM-Newton, BeppoSAX, CGRO, and SIGMA data showed that the hard X-rays spectrum is well described by an absorbed power law with $\Gamma=1.65 \pm 0.04$ and $N_{\mathrm{H}}=2.7 \times$ $10^{23} \mathrm{~cm}^{-2}$, with no indication of a cut-off or reflection component. The data presented here now show evidence for a cut-off at $80 \mathrm{keV}$ and $\Gamma=1.3$. Recently, a turn over at $E_{\mathrm{C}}=30 \pm$ $13 \mathrm{keV}$ with $\Gamma=0.9 \pm 0.3$ was also reported in $S u z a k u$ data of NGC 4388 (Shirai et al. 2008), detecting also significant spectral variability.

NGC 4507: this Seyfert 2 shows a reflection component of the order of $R=0.5-1$ in BeppoSAX observations and a photon index of $\Gamma=1.3-1.9$, while the cut-off energy was not constrained (Dadina 2007). The IBIS/ISGRI data do not require the presence of reflection, and a simple cut-off power law $(\Gamma=1.1 \pm$ $\left.0.2, E_{\mathrm{C}}=65_{-12}^{+27} \mathrm{keV}\right)$ is sufficient $\left(\chi^{2}=3.5\right.$ for 7 d.o.f. $)$. Applying the PEXRAV model without cut-off, we obtained a chi-squared of $\chi^{2}=6.2$ for 7 d.o.f. The value of the photon index and the reflection obtained are $\Gamma=1.7 \pm 0.1$ and $R=0.6_{-0.5}^{+1.5}$, respectively, consistent with the BeppoSAX observations.

NGC 4593: BeppoSAX data of this Seyfert 1 showed a reflection component with $R=1.1_{-0.5}^{+2.6}$ and $\Gamma=1.9 \pm 0.1$ but no evidence for a cut-off (Dadina 2007). From IBIS/ISGRI and JEM-X data we found that a power law with a cut-off gives a

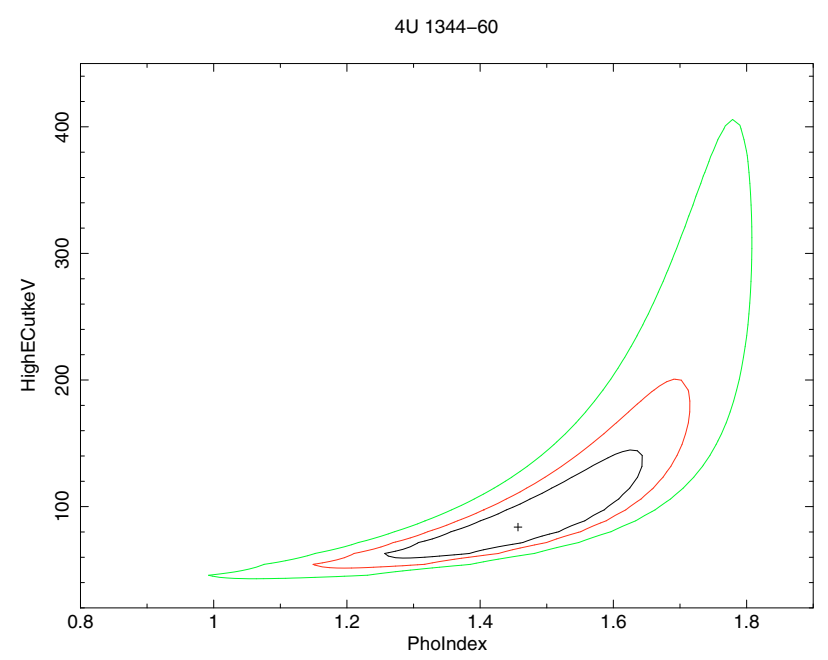

Fig. A.1. Contour plot of the 1-3 sigma confidence levels of cut-off energy $[\mathrm{keV}]$ versus photon index $\Gamma$. A steeper spectral fit requires a higher cut-off energy.

good fit to the data $\left(\Gamma=1.5 \pm 0.1, E_{\mathrm{C}}=193_{-93}^{+12} \mathrm{keV}, \chi^{2}=15\right.$ for 15 d.o.f.). The value of the absorption has been fixed to $N_{\mathrm{H}}=2 \times$ $10^{20} \mathrm{~cm}^{-2}$. Applying a PEXRAV model with no cut-off also provides a good representation of the data with $\chi^{2}=10.7$ for 15 d.o.f. and gives a photon index of $\Gamma=1.9 \pm 0.1$ and a reflection component of $R=1.7_{-0.8}^{+1.6}$, both parameters consistent with the results by Dadina et al. (2007).

NGC 4945: the IBIS/ISGRI spectrum of the Seyfert 2 galaxy NGC 4945 (with an exposure of $276 \mathrm{ks}$ ) is amongst the six analyzed by Soldi et al. (2005). In their work the best model to the data is a simple power law $\left(\Gamma=1.9_{-0.1}^{+0.1}\right)$, and they give a lower limit to the possible high energy cut-off, $E_{\mathrm{C}} \gg 130 \mathrm{keV}$. Using the new IBIS/ISGRI and JEM-X data we found that a simple absorbed power law does not provide a good fit $\left(\chi^{2}=30\right.$ for 12 d.o.f.) and a cutoff at high energy is necessary. This component improves significantly the goodness of the fit $\left(\chi^{2}=3.3\right.$ for 11 d.o.f.) and gives a photon index of $\Gamma=1.4_{-0.2}^{+0.2}$ and a cutoff at $E_{\mathrm{C}}=121_{-34}^{+63} \mathrm{keV}$. The absorption has been fixed to $N_{\mathrm{H}}=400 \times$ $10^{22} \mathrm{~cm}^{-2}$. These values are also consistent with those obtained by Guainazzi et al. (2000), using BeppoSAX data.

Cen A is the only extragalactic object also seen by IBIS/PICsIT. Combined IBIS/ISGRI, SPI, and PICsIT data analysis gave a spectral slope of $\Gamma=1.80 \pm 0.01$ (Lubiński 2009), close to the results presented here. It has been also detected by Fermi/LAT (Abdo et al. 2009) and at very high energy gamma-rays by HESS (Aharonian et al. 2009).

4 U 1344-60: in Beckmann et al. (2006) the analysis of combined XMM-Newton and INTEGRAL data showed a good representation of the broad-band data with an absorbed power-law $(\Gamma=1.65)$ plus a Gaussian component. Applying the same simple model to the IBIS/ISGRI data used here, leads to a steep hard X-ray power law $(\Gamma=1.9 \pm 0.1)$ but gives a bad fit result $\left(\chi_{v}^{2}>\right.$ 2). Using the simultaneous JEM-X and ISGRI data and adding a cut-off, flattens the spectrum: the lower the cut-off energy, the flatter the resulting photon index $\left(\Gamma=1.5_{-0.2}^{+0.1}, E_{\mathrm{C}}=86_{-22}^{+31}\right)$, as can be seen in Fig. A.1. Panessa et al. (2008) analysed again non-simultaneous XMM-Newton and INTEGRAL data, finding a cut-off power law with photon index $\Gamma=1.75_{-0.14}^{+0.18}$ and $E_{\mathrm{C}}>$ $78 \mathrm{keV}$. Fixing the spectral slope for the combined JEM-X and IBIS/ISGRI spectrum to $\Gamma=1.75$, we also get a higher cut-off energy of $E_{\mathrm{C}}=214_{-44}^{+73} \mathrm{keV}$. 
IC 4329A: the BeppoSAX spectrum of this Seyfert 1 analysed by Dadina (2007) by applying a PEXRAV model showed in several observations a cut-off at energies $E_{\mathrm{C}}>120 \mathrm{keV}$, with a reflection component of $R=0.5-1.5$ and a photon index of $\Gamma=1.9-2.0$. Using the same model for the combined JEM-X and ISGRI data, we get a flatter spectrum, with $\Gamma=1.6 \pm 0.3$, $E_{\mathrm{C}}=124_{-48}^{+243} \mathrm{keV}$ and $R=0.3_{-0.3}^{+0.8}$, and thus a spectrum which is consistent with no reflection component. We therefore applied the simpler cut-off power law model which leads to $\Gamma=1.4 \pm$ 0.1 and $E_{\mathrm{C}}=86_{-13}^{+18} \mathrm{keV}$.

Circinus Galaxy: the combined IBIS/ISGRI and SPI spectrum of the Circinus galaxy has been studied by Soldi et al. (2005) based on $589 \mathrm{ks}$ exposure time, finding as the best model to the data an absorbed power law $\left(\Gamma=1.8_{-0.5}^{+0.4}\right)$ with $N_{\mathrm{H}}=400 \times$ $10^{22} \mathrm{~cm}^{-2}$ and a high-energy cutoff at $E_{\mathrm{C}}=50_{-18}^{+51} \mathrm{keV}$, values consistent with the ones obtained by previous BeppoSAX observations. Using the same model and fixing the hydrogen column density to the one used by Soldi et al. (2005), we found the best fit to the data $\left(\chi^{2}=16\right.$ for 11 d.o.f.), with parameters consistent with the values listed above $\left(\Gamma=1.2 \pm 0.2, E_{\mathrm{C}}=30 \pm\right.$ $3 \mathrm{keV}$ ). Dadina (2007) analysed two BeppoSAX observations applying reflection models with $\Gamma_{1}=1.7 \pm 0.1, R_{1}=0.29_{-0.04}^{+0.05}$ and $\Gamma_{2}=1.3 \pm 0.2, R_{2}=0.35_{-0.09}^{+0.39}$, both with cut-off at $E_{\mathrm{C}} \lesssim 50 \mathrm{keV}$. Adding a reflection component to the data presented here improves significantly the fit $\left(\chi^{2}=6.1\right.$ for 9 d.o.f. $)$ but the parameter are not well constrained: $\Gamma=1.8_{-0.6}^{+0.4}, E_{\mathrm{C}}=$ $76_{-30}^{+180} \mathrm{keV}$ and $R=1.6_{-1.1}^{+8.0}$.

PG 1416-129: the Seyfert 1 galaxy had been included in the first INTEGRAL AGN catalogue (Beckmann et al. 2006a). Subsequent analysis of the data with improved software showed that the detection of this source was indeed spurious, and we now do not consider this source to be an INTEGRAL detected object.

IGR J16351-5806: this Seyfert 2 galaxy has recently been claimed to be a Compton thick AGN with $N_{\mathrm{H}}>1.5 \times 10^{24} \mathrm{~cm}^{-2}$ (Malizia et al. 2009). This is based on the observation that the hard X-ray spectrum is rather flat, as also shown here with $\Gamma=1.5$, and that a strong iron $\mathrm{K} \alpha$ line with $E W>1 \mathrm{keV}$ is an indicator for a significant reflection component. Malizia et al. fit the spectrum therefore as a pure reflection spectrum. As the ISGRI data alone do not allow fitting the complex model, we assumed the simple model of an unabsorbed power law, as also observed in Swift/XRT data (Landi et al. 2007a).

IGR J16426+6536: this narrow-line Seyfert $1(z=0.323 \pm$ 0.001, Parisi et al. 2008; Butler et al. 2009) shows a rather high luminosity of $L_{(20-100 \mathrm{keV})}=2 \times 10^{46} \mathrm{erg} \mathrm{s}^{-1}$ but moderate mass of the central black hole $\left(M=1.1 \times 10^{7} M_{\odot}\right)$. This leads to a large Eddington ratio of about $\lambda \simeq 90$ (defined in Sect. 4.2). It has to be pointed out though, that the soft X-ray counterpart as detected by XMM-Newton exhibits only a flux of $1.1 \times$ $10^{-12} \mathrm{erg} \mathrm{cm}^{-2} \mathrm{~s}^{-1}$ (Ibarra et al. 2008), about 10 times below what would be expected from the INTEGRAL detection with $f_{(20-40 \mathrm{keV})}=2.6 \times 10^{-11} \mathrm{erg} \mathrm{cm}^{-2} \mathrm{~s}^{-1}$. This might indicate that this source is strongly absorbed, highly variable, or that the optical counterpart is a misidentification, leading to the high superEddington accretion rate.

GRS 1734-292: the IBIS/ISGRI data of GRS 1734-292 (for a total exposure of $4040 \mathrm{ks}$ ) have been analyzed by Molina et al. (2006), along with $A S C A / G I S$ data, who found that the best fit is obtained using an absorbed cut-off power law. Using the new IBIS/ISGRI and JEM-X data we found that the best model $\left(\chi^{2}=12.1\right.$ for 11 d.o.f.) is an absorbed power-law with a cutoff and a reflection component with the following parameters: $N_{\mathrm{H}}=3.7 \times 10^{22} \mathrm{~cm}^{-2}, E_{\mathrm{C}}=220_{-150}^{+200} \mathrm{keV}, \Gamma=2.0_{-0.4}^{+0.3}$ and
$R=2.7_{-1.8}^{+3.1}$. The black hole mass of GRS 1734-292 has been provided by I. Papadakis (private communication) based on the empirical relation found by Tremaine et al. (2002) between the black hole mass and the stellar velocity dispersion $\sigma_{\mathrm{s}}$, estimating $\sigma_{\mathrm{s}}$ from the width of the [O III] line reported by Marti et al. (1998).

IGR J17488-3253: for this Seyfert 1 galaxy $(z=0.02)$ a fit of simultaneous JEM-X and IBIS/ISGRI data by a cut-off power law model results in $\chi_{v}^{2}=5.8$ for 11 degrees of freedom. As the source is located in a dense area, it is possible that the JEM-X data are contaminated by sources within the field of view, especially as the JEM-X data are well represented by a black body model with a temperature of $0.7 \mathrm{keV}$. We therefore analysed Swift/XRT data of the source. A combined fit of XRT and ISGRI data results in $\chi_{v}^{2}=1.1$ for 119 degrees of freedom, showing an absorbed power law model with $N_{\mathrm{H}}=0.72 \pm 0.04$ and $\Gamma=1.63 \pm 0.03$, representing the data well over the $0.3 \mathrm{keV}$ to $100 \mathrm{keV}$ energy range.

QSO B1957+405 (Cyg A): the IBIS/ISGRI (together with BeppoSAX/MECS and PDS) spectrum of Cygnus A has been analyzed by Molina et al. (2006), using data for a total exposure of $426 \mathrm{ks}$. In their work they fitted the high-energy spectrum with a complex model in order to take into account also the gas emission of the galaxy cluster to which the AGN belongs. For doing so they used an absorbed power law plus a bremsstrahlung component. Following their work we fitted the IBIS/ISGRI and JEM-X spectrum using the same model (plus a cross-calibration constant) and we found that it provides the best fit $\left(\chi^{2}=6\right.$ for 9 d.o.f.) and that the parameters obtained $\left(\Gamma=1.9_{-0.2}^{+0.1}\right.$ and $k T=$ $4.5_{-2.7}^{+3.7} \mathrm{keV}$ ) are in good agreement with those they obtained. The value of the absorption has been fixed to $N_{\mathrm{H}}=2 \times 10^{23} \mathrm{~cm}^{-2}$.

IGR J21247+5058: the high-energy broad-band spectrum of this radio galaxy was obtained by Molina et al. (2007) by combining XMM-Newton and Swift/XRT observation with IBIS/ISGRI data. The $0.4-100 \mathrm{keV}$ spectrum is well described by a power law, with slope $\Gamma=1.5$, characterized by complex absorption due to two layers of material partially covering the source and a high-energy cut-off around 70-80 keV, consistent with our findings $\left(\Gamma=1.4 \pm 0.1, E_{\mathrm{C}}=61_{-11}^{+22} \mathrm{keV}\right)$ using JEM-X and ISGRI data. As Molina et al. point out, features such as a narrow iron line and a Compton reflection component, if present, are weak, suggesting that reprocessing of the power-law photons in the accretion disc plays a negligible role in the source.

\section{References}

Abdo, A. A., Ackermann, M., Ajello, M., et al. 2009, ApJ, 700, 597 Abramowicz, M. A., Chen, X.-M., Granath, M., \& Lasota, J.-P. 1996, ApJ, 471, 762

Aharonian, F., Akhperjanian, A. G., Anton, G., et al. 2009, ApJ, 695, L40 Antonucci, R. 1993, ARA\&A, 31, 473

Arnaud, K. A. 1996, in Astronomical Data Analysis Software and Systems V, ed. G. Jacoby, \& J. Barnes, ASP Conf. Ser., 101, 17

Awaki, H., Koyama, K., Inoue, H., \& Halpern, J. P. 1991, PASJ, 43, 195 Awaki, H., Murakami, H., Leighly, K. M., et al. 2005, ApJ, 632, 793

Barthelmy, S. D., Barbier, L. M., Cummings, J. R., et al. 2005, SSRv, 120, 143 Bassani, L., Dadina, M., \& Maiolino, R. 1999, ApJS, 121, 473

Bassani, L., Molina, M., Malizia, A., et al. 2006, ApJ, 636, L65

Bassani, L., Landi, R., \& Malizia, A. 2007, ApJ, 669, L1

Beckmann, V., Gehrels, N., Shrader, C. R., \& Soldi, S. 2006a, ApJ, 638, 642 Beckmann, V., Soldi, S., Shrader, C. R., Gehrels, N., \& Produit, N. 2006b, ApJ, 652,126

Beckmann, V., Soldi, S., Shrader, C. R., \& Gehrels, N. 2006c, The X-ray Universe 2005, 604, 777

Beckmann, V., Gehrels, N., \& Tueller, J. 2007a, ApJ, 666, 122 
Beckmann, V., Petry, D., \& Weidenspointner, G. 2007b, ATel, 1264

Beckmann, V., Ishibashi, W., Bottacini, E., Ajello, M., \& Greiner, J. 2007c, ATel, 1317

Beckmann, V., Courvoisier, T. J.-L., Gehrels, N., et al. 2008, A\&A, 492, 93

Beckmann, V., Courvoisier, T. J.-L., Gehrels, N., et al. 2009, AIP Conf. Ser., 1126,141

Bian, W., \& Gu, Q. 2007, ApJ, 657, 159

Bianchi, S., Bonilla, N. F., Guainazzi, M., et al. 2009, A\&A, 501, 915

Bodaghee, A., Courvoisier, T. J.-L., Rodriguez, J., et al. 2007, A\&A, 467, 585

Butler, S. C., Tomsick, J. A., Chaty, S., et al. 2009, ApJ, 698, 502

Corbel, S., Fender, R. P., Tzioumis, A. K., et al. 2000, A\&A, 359, 251

Corbel, S., Nowak, M. A., Fender, R. P., et al. 2003, A\&A, 400, 1007

Courvoisier, T. J.-L., \& Türler, M. 2005, A\&A, 444, 417

Courvoisier, T. J.-L., Walter, R., Beckmann, V., et al. 2003, A\&A, 411, L53

Dadina, M. 2007, A\&A, 461, 1209

Dadina, M. 2008, A\&A, 485, 417

Deluit, S., \& Courvoisier, T. J.-L. 2003, A\&A, 399, 77

de Rosa, A., Piro, L., Perola, G. C., et al. 2007, A\&A, 463, 903

Domingo, A., Caballero, M. D., Figueras, F., et al. 2003, A\&A, 411, L281

Ebisawa, K., Bourban, G., Bodaghee, A., Mowlavi, N., \& Courvoisier, T. J.-L. 2003, A\&A, 411, L59

Eckert, D., \& Paltani, S. 2009, A\&A, 495, 415

Fabian, A. C., Vasudevan, R. V., \& Gandhi, P. 2008, MNRAS, 385, L43

Fabian, A. C., Vasudevan, R. V., Mushotzky, R. F., Winter, L. M., \& Reynolds, L. M. W. C. S. 2009, MNRAS, 349, L89

Fender, R., Körding, E., Belloni, T., et al. 2007, Proc. of Science, 6th Microquasar Workshop [arXiv: 0706. 3838]

Ferrarese, L., \& Merritt, D. 2000, ApJ, 539, L9

Gehrels, N., Chincarini, G., Giommi, P., et al. 2004, ApJ, 611, 1005

Gierliński, M., Nikolajuk, M., \& Czerny, B. 2008, MNRAS, 383, 741

Gilli, R., Comastri, A., \& Hasinger, G. 2007, A\&A, 463, 79

Goncalves, T. S., Martin, D. C., Halpern, J. P., Eracleous, M., \& Pavlov, G. G. 2009, ATel, 1623

Gondoin, P., Orr, A., \& Lumb, D. 2003, A\&A, 398, 967

Gondek, D., Zdziarski, A. A., Johnson, W. N., et al. 1996, MNRAS, 282, 646

Graham, A. W. 2008, PASA, 25, 167

Greene, J. E. \& Ho, L. C. 2005, ApJ, 627, 721

Greenhill, L. J. 1997, ASPC, 113, 394

Guainazzi, M., Matt, G., Brandt, W. N., et al. 2000, A\&A, 356, 463

Guilbert, P. W., \& Rees, M.-J. 1988, MNRAS, 233, 475

Hicks, E. K. S., \& Malkan, M. A. 2008, ApJS, 174, 31

Ibarra, A., Kuulkers, E., \& Saxton, R. 2008, ATel, 1397

Johnson, W. N., Kinzer, R. L., Kurfess, J. D., et al. 1993, ApJS, 86, 693

Kaspi, S., Smith, P. S., Netzer, H., et al. 2000, ApJ, 533, 631

Kirsch, M. G., Briel, U. G., Burrows, D., et al. 2005, SPIE, 5898, 22

Landi, R., Masetti, N., Gehrels, N., et al. 2007a, ATel, 990

Landi, R., Malizia, A., Masetti, N., et al. 2007b, ATel, 1274

Landi, R., Masetti, N., Stephen, J. B., et al. 2007c, ATel, 1288

Landi, R., Masetti, N., Sguera, V., et al. 2007d, ATel, 1322

Landi, R., Stephen, J. B., Masetti, N., et al. 2009, A\&A, 493, 893

Laor, A. 2000, New. Astron. Rev., 44, 503

Lawrence, A., \& Elvis, M. 1982, ApJ, 256, 410

Lebrun, F., Leray, J. P., Lavocat, P., et al. 2003, A\&A, 411, L141

Lubiński, P. 2009, A\&A, 496, 557

Lubiński, P., Walter, R., Beckmann, V., et al. 2009, in prep.

Lund, N., Budtz-Jørgensen, C., Westergaard, N. J., et al. 2003, A\&A, 411, L231

Magdziarz, P., \& Zdziarski, A. A. 1995, MNRAS, 273, 837

Malizia, A., Bassani, L., Stephen, J. B., et al. 2003, ApJ, 589, L17

Malizia, A., Landi, R., Bassani, L., et al. 2007, ApJ, 668, 81

Malizia, A., Bassani, L., Bird, A. J., et al. 2008, MNRAS, 389, 1360

Malizia, A., Bassani, L., Panessa, F., de Rosa, A., \& Bird, A. J. 2009, MNRAS, 349, L121
Marti, J., Mirabel, I. F., Chaty, S., \& Rodriguez, L. F. 1998, A\&A, 330, 72 Masetti, N., Morelli, L., Palazzi, E., et al. 2006, A\&A, 459, 21 Masetti, N., Parisi, P., Palazzi, E., et al. 2009, A\&A, 495, 121

Mas-Hesse, J. M., Giménez, A., Culhane, J. L., et al. 2003, A\&A, 411, L261

Merloni, A., Heinz, S., \& Di Matteo, T. 2003, MNRAS, 345, 1057

Middleton, M., Done, C., \& Schurch, N. 2008, MNRAS, 383, 1501

Molina, M., Malizia, A., Bassani, L., et al. 2006, MNRAS, 371, 821

Molina, M., Bassani, L., Malizia, A., et al. 2008, MNRAS, 390, 1217

Morales, R., \& Fabian, A. C. 2002, MNRAS, 329, 209

Murphy, K. D., \& Yaqoob, T. 2009, MNRAS, 397, 1549

Nagar, N. M., Oliva, E., Marconi, A., \& Maiolino, R. 2002, A\&A, 391, L21

Novak, G. S., Faber, S. M., \& Dekel, A. 2006, ApJ, 637, 96

O’Dell, S. L., Puschell, J. J., Stein, W. A., \& Warner, J. W. 1978, ApJS, 38, 267

O'Dowd, M., Urry, C. M. \& Scarpa, R. 2002, ApJ, 580, 96

Paltani, S., \& Türler, M. 2005, A\&A, 435, 811

Paltani, S., Walter, R., McHardy, I. M., et al. 2008, A\&A, 485, 707

Panessa, F., Bassani, L., de Rosa, A., et al. 2008, A\&A 467, 519

Pappa, A., Georgantopoulos, I., Stewart, G. C., \& Zezas, A. L. 2001, MNRAS, 326, 995

Parisi, P., Masetti, N., Malizia, A., et al. 2008, ATel, 1800

Peterson, B. M., Ferrarese, L., Gilbert, K. M., et al. 2004, ApJ, 613, 682

Remillard, R. A., \& McClintock, J. E. 2006, ARA\&A, 44, 49

Revnivtsev, M., Sunyaev, R., Lutovinov, A., \& Sazonov, S. 2007, ATel, 1253

Ricci, C., Beckmann, V., Courvoisier, T. J.-L., et al. 2009a, in prep.

Ricci, C., Beckmann, V., Audard, M., \& Courvoisier, T. J.-L. 2009b, A\&A, submitted

Rodriguez, J., Tomsick, J. A., \& Chaty, S. 2008, A\&A, 482, 731

Sazonov, S., \& Revnivtsev, M. 2004, A\&A, 423, 469

Sazonov, S., Krivonos, R., Revnivtsev, M., Churazov, E., \& Sunyaev, R. 2007, A\&A, 462, 57

Sazonov, S., Revnivtsev, M., Burenin, R., et al. 2008, A\&A, 487, 509

Siemiginowska, A., Kuhn, O., Elvis, M., et al. 1995, ApJ, 454, 77

Shirai, H., Fukazawa, Y., Sasada, M., et al. 2008, PASJ, 60, 263

Simpson, G., \& Mayer-Hasselwander, H. 1986, A\&A, 162, 340

Smith, D. A., Georgantopoulos, I., \& Warwick, R. S. 2001, ApJ, 550, 635

Soldi, S., Beckmann, V., Bassani, L., et al. 2005, A\&A, 444, 431

Soldi, S., Türler, M., Paltani, S., et al. 2008, A\&A, 486, 411

Steffen, A. T., Barger, A. J., Cowie, L. L., Mushotzky, R. F., \& Yang, Y. 2003, ApJ, 596, L23

Terashima, Y., Gallo, L. C., Inoue, H., et al. 2009, PASJ, 61, 299

Tomsick, J. A., Chaty, S., Rodriguez, J., Walter, R., \& Kaaret, P. 2008, ApJ, 685, 1143

Treister, E., \& Urry, C. M. 2005, ApJ, 630, 115

Treister, E., Urry, C. M., \& Virani, S. 2009, ApJ, 696, 110

Tremaine, S., Gebhardt, K., Bender, R., et al. 2002, ApJ, 574, 740

Tueller, J., Mushotzky, R. F., Barthelmy, S. D., et al. 2008, ApJ, 681, 113

Uttley, P., \& McHardy, I. M. 2005, MNRAS, 363, 586

Vestergaard, M. 2004, ASPC, 311, 69

Wandel, A. 2002, ApJ, 565, 762

Wang, J., Mao, Y. F., \& Wei, J. Y. 2009, AJ, 137, 3388

Winkler, C., Courvoisier, T. J.-L., Di Cocco, G., et al. 2003, A\&A, 411, L1

Winter, L. M., Mushotzky, R. F., Tueller, J., \& Markwardt, C. 2008, ApJ, 674, 686

Winter, L. M., Mushotzky, R. F., Reynolds, C. S., \& Tueller, J. 2009, ApJ, 690, 1322

Woo, J.-H., \& Urry, M. 2002, ApJ, 579, 530

Wu, X. B., Wang, R., Kong, M. Z., et al. 2004, A\&A, 424, 793

Zdziarski, A. A., Johnson, W. N., Done, C., Smith, D., \& McNaron-Brown, K. 1995, ApJ, 438, L63

Zdziarski, A. A., Lubiński, P., \& Smith, D. A. 1999, MNRAS, 303, L11

Zdziarski, A. A., Poutanen, J., \& Johnson, W. N. 2000, ApJ, 542, 703

Zurita Heras, J. A., Chaty, S., \& Tomsick, J. A. 2009, A\&A, 502, 787 